\title{
AIRWAY SMOOTH MUSCLE IN HEALTH AND DISEASE
}

\author{
by \\ Leslie Yee Mann Chin \\ BSc, The University of Calgary, 2005 \\ A THESIS SUBMITTED IN PARTIAL FULFILLMENT OF \\ THE REQUIREMENTS FOR THE DEGREE OF \\ DOCTOR OF PHILOSOPHY \\ in
}

The Faculty of Graduate Studies

(Pathology and Laboratory Medicine)

THE UNIVERSITY OF BRITISH COLUMBIA

(Vancouver)

AUGUST 2010

(C) Leslie Yee Mann Chin, 2010 


\section{ABSTRACT}

This thesis focuses on the structure and function of airway smooth muscle (ASM) in health and disease. By employing the use of structural analysis by electron microscopy, functional analysis by mechanical measurements, and biochemical analysis, this thesis provides valuable insight into ASM pathophysiology. The first two chapters focus on the mechanisms by which the contractile apparatus is arranged within the cell. The studies examined whether the actin filament lattice acts a scaffold to facilitate myosin filament assembly within contractile units and the contractile response to potassium chloride $(\mathrm{KCl})$. The muscle was treated with cytochalasin $\mathrm{D}(\mathrm{CD})$, a known actin filament disrupter, but this provided little insight on whether the actin lattice guides myosin filament assembly, since CD had a limited effect on actin filaments but a significant effect on force. $\mathrm{KCl}$ was found to cause contraction of similar force to acetylcholine contraction, despite the presence of fewer myosin filaments. $\mathrm{KCl}$ likely caused depolymerization of myosin filaments upon activation and allowed for force generation by non-filamentous myosin molecules. In the last two studies, human ASM was sourced from the tracheas of whole lungs donated for medical research. From this tissue source it was shown that, unlike in previous human ASM studies, human muscle is similar to that of other mammalian species and capable of significant isotonic shortening. This finding lends support to the use of animal ASM models as a proxy for human ASM. This also was the first study to examine human ASM in the paradigm of mechanical plasticity, using in situ muscle length as a reference length instead of the traditional $\mathrm{L}_{\max }$, and was the first to demonstrate length adaptation in human ASM. The mechanical properties of asthmatic ASM were found to differ from those of non-asthmatic ASM at several key 
measurements. Asthmatic ASM was found to have an altered length-tension relationship, increased passive tension, and maintained force better in response to a mechanical perturbation than non-asthmatic ASM. This last finding provides a possible mechanism by which asthmatic airways are more resistant to the bronchodilating effects of deep inspiration. Force generating capacity, shortening extent and velocity were not different. 
TABLE OF CONTENTS

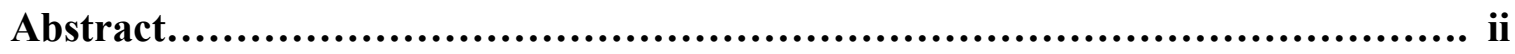

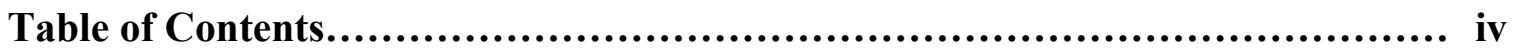

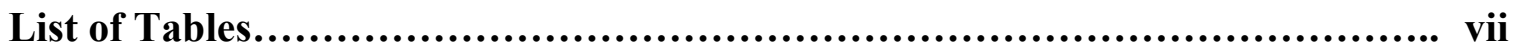

List of Figures.................................................................... viii

Acknowledgements.................................................................

Co-authorship Statement........................................................... xi

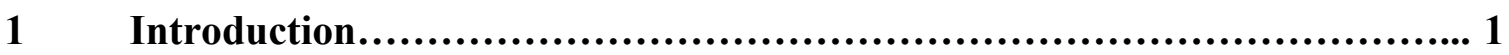

$1.1 \quad$ Thesis Description................................................... 1

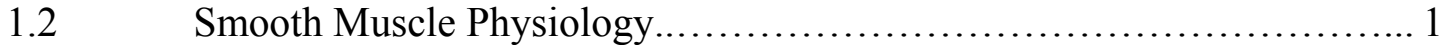

$1.3 \quad$ The Contractile Apparatus................................................. 3

1.4 Myosin Evanescence and Mechanical Plasticity.......................... 6

$1.5 \quad$ Smooth Muscle Signal Transduction.................................. 8

1.6 Myosin Filament Assembly ........................................ 10

$1.7 \quad$ Airway Smooth Muscle Function......................................... 11

$1.8 \quad$ Asthma and Airway Smooth Muscle..................................... 13

$1.9 \quad$ Experimental Summary .................................................... 15

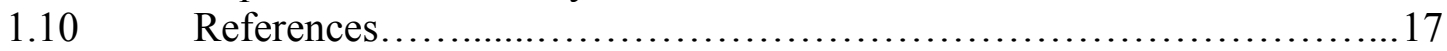

2 Facilitation of myosin filamentogenesis by the actin filament................. 23 lattice

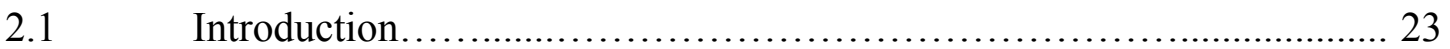

$2.2 \quad$ Materials and Methods..................................................... 27

2.2.1 Tissue Preparation and Equilibration..................................... 27

2.2.2 Protocol 1: Treatment with $1 \mu \mathrm{M}$ Cytochalasin D.......................... 28

2.2.3 Protocol 2: Treatment with $5 \mu \mathrm{M}$ Cytochalasin D........................... 28

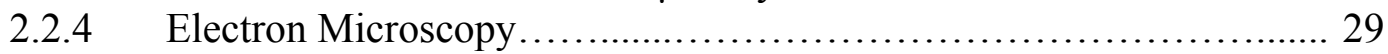

2.2.5 Morphometric Analysis............................................... 30

2.2.6 Atomic Force Microscopy and Myosin Filament Formation in vitro.... 31

2.2.7 Statistical Analysis.................................................... 32

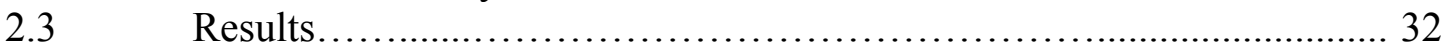

2.3.1 Protocol 1: Treatment with $1 \mu \mathrm{M}$ Cytochalasin D.......................... 32

2.3.1.1 Force Response to $1 \mu \mathrm{M}$ Cytochalasin................................ 32

2.3.1.2 Actin and Myosin Filament Density............................... 33

2.3.2 Protocol 2: $5 \mu \mathrm{M}$ Cytochalasin and Length Oscillation.................... 39

2.3.2.1 Force Response to $5 \mu \mathrm{M}$ Cytochalasin............................... 39

2.3.2.2 Actin and Myosin Filament Density................................. 40

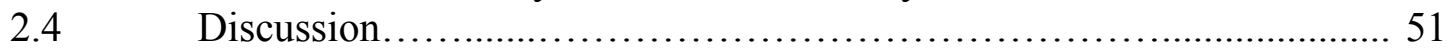

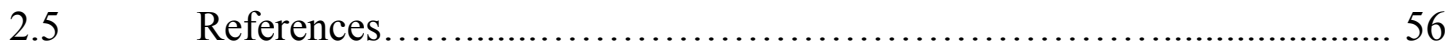


3 The number of myosin filaments in potassium chloride stimulated smooth muscle does not correlate to the force response

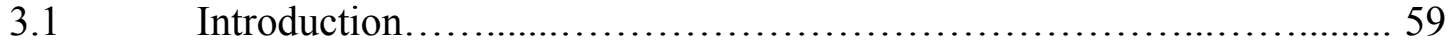

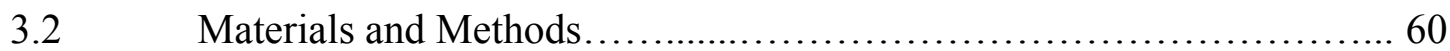

3.2.1 Tissue Preparation and Equilibration..................................... 60

3.2.2 Stimulation with Potassium Chloride or Acetylcholine..................... 62

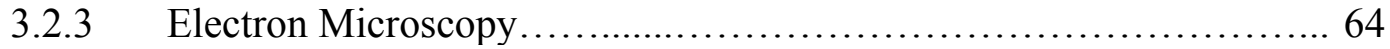

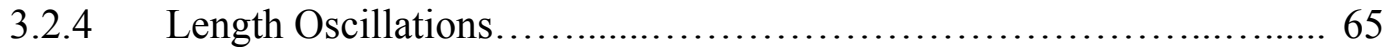

3.2.5 Determining Myosin Light Chain Phosphorylation.............................. 66

3.2.6 Morphometric and Statistical Analysis................................... 67

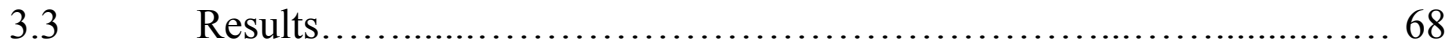

3.3.1 Force Response and Myosin Filament Density ........................ 68

3.3.2 Length Oscillation and Recovery...................................... 73

3.3.3 Measurement of rMLC Phosphorylation............................... 79

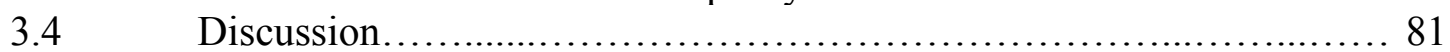

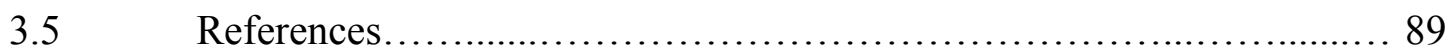

$4 \quad$ Human airway smooth muscle is structurally and mechanically.............. 93 similar to that of other species

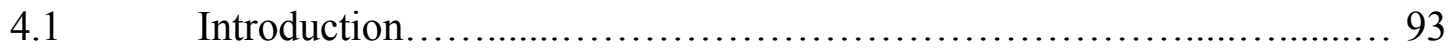

4.2 Materials and Methods.................................................. 94

4.2.1 Tissue Preparation and Equilibration.................................... 94

4.2.2 Mechanical Measurements.............................................. 97

4.2.3 Histology and Electron Microscopy.................................... 98

4.2.4 Statistical Analysis................................................... 98

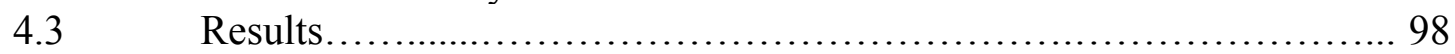

4.3.1 Muscle Bundle Properties and Morphology............................... 98

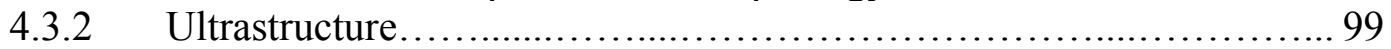

4.3.3 Force-Length Properties and Length Adaptation......................... 103

4.3.4 Recovery from Mechanical Perturbation.................................. 107

4.3.5 Shortening Velocity................................................. 107

4.3.6 Maximal Isotonic Shortening .................................. 112

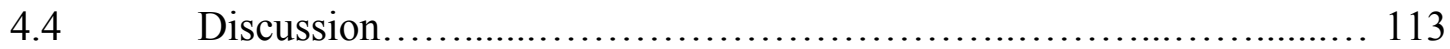

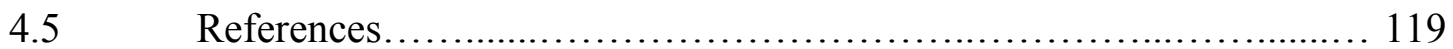

5 Mechanical properties of asthmatic airway smooth muscle revisited........ 123

$5.1 \quad$ Introduction...................................................... 123

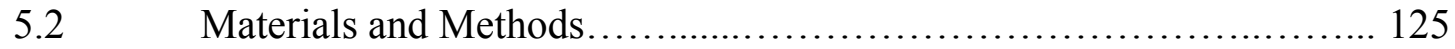

5.2.1 Tissue Preparation and Equilibration................................... 125

5.2.2 Mechanical Measurements............................................ 127

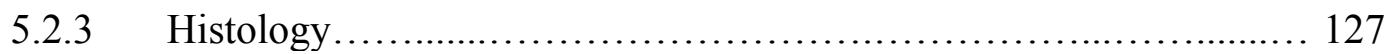

5.2.4 Statistical Analysis............................................. 128

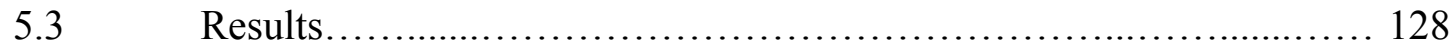

5.3.1 Muscle Bundle Properties............................................ 128

5.3.2 Length-Force Properties and Length Adaptation...................... 130

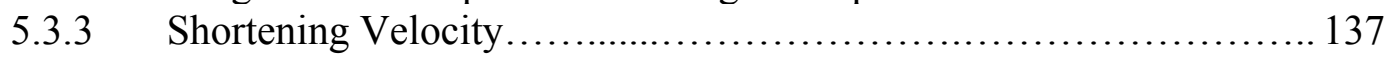


5.3.4 Maximal Isotonic Shortening...................................... 141

5.3.5 Recovery from Mechanical Perturbation............................................. 143

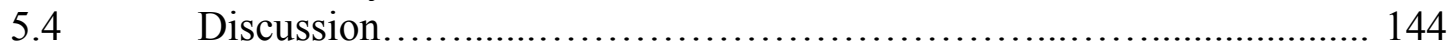

5.4.1 Does Asthmatic ASM Produce More Force? ........................... 145

5.4.2 Is the Force-Length Relationship Different in Asthmatics?................ 146

5.4.3 Does Asthmatic ASM Shorten Faster or More Than...................... 146

5.4.4 Does Asthmatic ASM Respond Differently to Mechanical .............. 149 Perturbation?

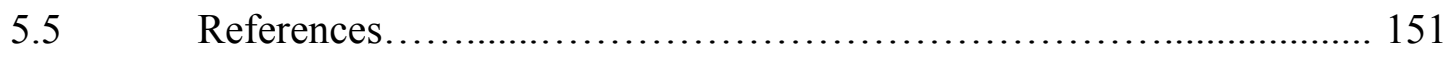

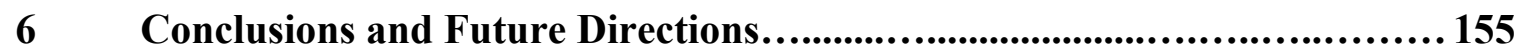

6.1 Myosin Filamentogenesis and the Contractile Apparatus.................. 155

6.2 Human ASM and Asthma Pathogenesis................................. 158

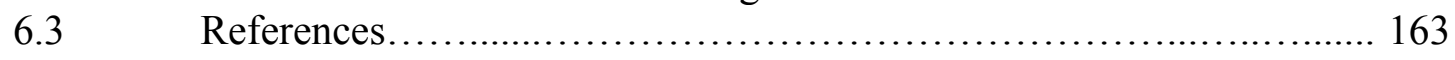

Appendix A: Ethics Approval ......................................................................164 


\section{LIST OF TABLES}

Table 4.1 Subject demographics and clinical details......................... 96

Table 5.1 Subject demographics and clinical details......................... 126 


\section{LIST OF FIGURES}

Figure 1.1 Transverse electron micrograph of a relaxed airway smooth................ 4 muscle cell

Figure 1.2 Recovery of force and thick filament density following..................... 8 length oscillation

Figure 2.1 Hypothetical model of myosin filament formation facilitated by the......... 26 actin filament lattice

Figure 2.2 Force response to $1 \mu \mathrm{M}$ cytochalasin D................................... 34

Figure 2.3 Electron micrograph of a cell treated with $1 \mu \mathrm{M}$ cytochalasin D..............35

Figure 2.4 Actin filament density of smooth muscle treated with either the............ 36 vehicle control (VC) or $1 \mu \mathrm{M}$ cytochalasin $\mathrm{D}(\mathrm{CD})$

Figure 2.5 Myosin filament density of smooth muscle treated with either the 38 vehicle control (VC) or $1 \mu \mathrm{M}$ cytochalasin $\mathrm{D}(\mathrm{CD})$

Figure 2.6 Treatment with $5 \mu \mathrm{M}$ cytochalasin D during length oscillation..............42 and recovery

Figure 2.7 Myosin filaments polymerized in vitro imaged by atomic 43 force microscopy

Figure 2.8 EM image processing for computerized actin filament counting........... 45

Figure 2.9 Actin filament density of smooth muscle treated with either the........... 48 vehicle control (VC) or $5 \mu \mathrm{M}$ cytochalasin $\mathrm{D}$ (CD)

Figure 2.10 Myosin filament density of smooth muscle treated with either the ..........50 vehicle control (VC) or $5 \mu \mathrm{M}$ cytochalasin $\mathrm{D}$ (CD)

Figure 3.1 Determination of $\mathrm{KCl}$ concentration....................................64

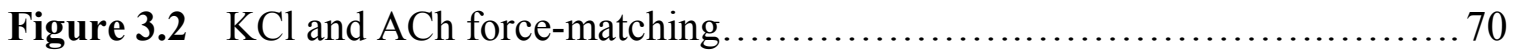

Figure 3.3 Cross-sectional electron micrograph of a $\mathrm{KCl}$ contracted cell............. 71

Figure 3.4 Cross-sectional electron micrograph of an ACh contracted cell............ 72

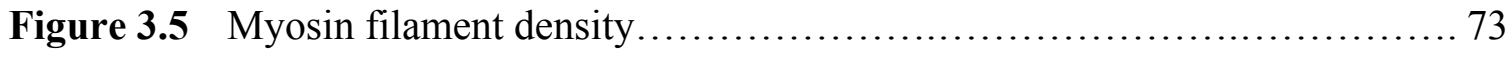

Figure 3.6 Sample force trace of an ASM strip with length oscillation and recovery... 75

Figure 3.7 Length oscillation.................................................... 76

Figure 3.8 Force peaks from the stretch cycle of the length oscillation.............. 77 
Figure 3.9 Recovery after length oscillation................................ 78

Figure 3.10 Adjusted recovery after length oscillation........................... 79

Figure 3.11 Myosin light chain phosphorylation................................ 80

Figure 3.12 Contractile apparatus model of $\mathrm{KCl}$ and $\mathrm{ACh}$ activation................. 85

Figure 4.1 Histological section of airway smooth muscle....................... 100

Figure 4.2 Transverse electron micrograph of an ASM cell....................... 101

Figure 4.3 Electron micrograph of a longitudinal ASM cell section................. 102

Figure 4.4 Isometric force at different lengths................................ 105

Figure 4.5 Active and passive force-length relationships before and.............. 108 after length adaptation

Figure 4.6 Isometric force recovery following a length oscillation................ 109

Figure 4.7 Determining shortening velocity during an isotonic contraction............110

Figure 4.8 Isotonic shortening velocity measured against different loads at two..... 111 time points

Figure 4.9 Maximal shortening............................................. 112

Figure 5.1 Asthmatic airway smooth muscle morphology $\ldots \ldots \ldots \ldots \ldots \ldots \ldots \ldots \ldots . \ldots \ldots$

Figure 5.2 Comparison of maximal stress generation......................... 130

Figure 5.3 Time course of length changes and the resulting active and passive.......133 force

Figure 5.4 Active force-length relationship before and after length adaptation........135

Figure 5.5 Passive force-length relationship before and after length adaptation...... 136

Figure 5.6 Isotonic shortening velocity, individual data points at the early release... 138

Figure 5.7 Isotonic shortening velocity, individual data points at the late release.... 139

Figure 5.8 Averaged isotonic shortening velocities to various loads, early...........140 phase release.

Figure 5.9 Averaged isotonic shortening velocities to various loads, late phase release.

Figure 5.10 Maximal isotonic shortening.................................. 142

Figure 5.11 Response to mechanical perturbation............................ 144 


\section{ACKNOWLEDGEMENTS}

I am forever in indebted to the wonderful people I have met at the University of British Columbia. They have made this an incredibly enjoyable experience by providing entertainment, knowledge, and constant support. I would like to particularly thank my supervisor Dr. Chun Seow, who has guided me along this journey. Without the mentoring of Dr. Seow I would not have been able to develop my scientific curiosity as I have and would be hopelessly unfocused. He has provided answers to all of my many questions and has always been available to me. In his lab I have met many great people including Dr. Ynuk Bossé and Farah Ali who have been dear friends, in addition to supporting me with my research.

Also, I would like to thank my committee members who have been so facilitative and critically engaged in my research: Dr. David Granville, Dr. Thomas Podor, Dr. Decheng Yang, and Dr. Peter Paré. Dr. Paré has been a remarkable mentor, from whom I have learned so much - a supervisor who was not officially a supervisor - and someone that I deeply admire. I have the most profound gratitude for all of my mentors, professors, and teachers who have taught me along the way and the support people, technicians, and administrators who have assisted me.

I have met many great people during my time at UBC who have brought joy and emotional support along the way. I must thank Marie France Witty, Amanda Vanden Hoek, Anna Meredith, and the many others in Pathology. Finally, this experience would not have been possible without Jennifer Pickering and my loving parents and sister who have always supported me and taught me how to be myself. 


\section{CO-AUTHORSHIP STATEMENT}

All of the work presented here was primarily led by me. The manuscript, experiments, and data analysis were all written and performed by me, with some help in certain circumstances. The manuscript was graciously edited by my committee members, Dr. Chun Seow, Dr. Decheng Yang, Dr. David Granville, and Dr. Peter Paré. As for the experiments, in Chapter Two, the atomic force microscopy and in vitro myosin filament polymerization was done by Kelvin Ip and Dennis Solomon. In Chapter Three, pilot electron microscopy work was begun by Dr. Yuekan Jiao and the protocol was optimized by Dr. Kuo-Hsing Kuo. All of the electron microscopy data presented was generated from tissue that was fixed and processed by me. Dr. Julian Solway provided research ideas to Chapter Three. In Chapters Two and Three, the tissue was provided from Pitt Meadow Meats Ltd. and obtained by Dennis Solomon.

In Chapters Four and Five, the experiments were done with the assistance of Dr. Ynuk Bossé, who also provided help with the data analysis and interpretation. Dr. Bossé also helped with the development of the research aims and experimental design. The tissue received from the International Institute for the Advancement of Medicine was arranged by Dr. Tillie Hackett, who processed the whole lung and provided us with the trachea. Stephanie Warner and Dorota Stefanowicz also provided assistance with the tissue. In Chapters Four and Five, the histological processing and staining was performed by Dennis Solomon, but the images were analyzed by me. In all of the experiments Dr. Chun Seow and Dr. Peter Paré were instrumental in guiding the experimental design and the research aims. 


\section{CHAPTER 1. INTRODUCTION}

\subsection{Thesis Description}

This thesis focuses on the structure and function of airway smooth muscle (ASM) at the subcellular, cellular and tissue levels. The aim of this work was to further elucidate aspects of ASM cell physiology pertaining to the cell's contractile apparatus and reexamine the role of ASM in the pathogenesis of asthma. The first two chapters describe studies designed to investigate the mechanism by which ASM cells generate force, specifically, how the subcellular structures are organized to produce contraction and the phenomena governing contractile filament organization. The last two chapters describe studies designed to examine the functional role of ASM in the pathogenesis of asthma, specifically, outlining the functional capabilities of human ASM compared to other mammalian ASM models and revisiting the question of whether asthmatic ASM is functionally different from non-asthmatic ASM.

\subsection{Smooth Muscle Physiology}

Smooth muscle cells are present in many diverse physiological systems and often times have important homeostatic functions. Unlike skeletal muscle, smooth muscle activation is entirely involuntary and controlled by the autonomic nervous system. Smooth muscle cells are responsible for focusing the lens of the eye, controlling bladder volume, peristalsis of the digestive tract, the pilomotor reflex in the skin, regulating blood pressure in the circulatory system, and contracting the uterus during childbirth. 
Mysteriously, as will be explained further below, the airways are also lined with smooth muscle cells. While smooth muscle organization and contractile function have been studied in detail for over sixty years, there are still many questions as to how the subcellular structures are organized to produce force and the mechanisms that regulate this contraction.

Much of our understanding about smooth muscle contraction has been adopted from functional studies of skeletal muscle [1-4]. This "borrowing" of knowledge is largely due to the lack of progress in elucidating the structural basis of contraction in smooth muscle which in turn is largely due to the lack of structural information about the contractile apparatus, because the cell's subcellular structure is amorphously 'smooth.' Investigators of skeletal and cardiac muscle have had the benefit of observing striation patterns in the cells to determine how the contractile machinery of those muscle cells is organized, as, the striation patterns are visualizations of the basic contractile units - the sarcomeres. These banding patterns are observed because the proteins involved in contraction, actin and myosin filaments reflect varying degrees of light. Under the microscope these bands were named the A-band, for anisotropic, which is predominately created by the myosin thick filaments, and the I-band, for isotropic, which is composed of actin thin filaments in the absence of myosin filaments. The presence of these bands allowed AF Huxley [5] and HE Huxley [6] to determine that muscle cells contract through a sliding filament mechanism. By observing skeletal muscle contraction under interference and phase contrast microscopy, they were able to independently show that the A-band does not change width during contraction, instead both sets of filaments slide pass one another, 
causing sarcomere narrowing. This mechanism of filament sliding was further refined with the discovery of myosin crossbridges, the molecular motors which, through ATP hydrolysis, pull actin filaments with a ratcheting motion [5].

Both of these mechanisms, the sliding filament and crossbridge rotation theories of contraction, are also thought to be responsible for smooth muscle contraction. However, unlike in skeletal muscle, the organization of contractile units in smooth muscle has never been established and the evidence of their existence and structural arrangement are largely supported only by functional data.

\subsection{The Contractile Apparatus}

Skeletal muscle, unlike smooth muscle, when examined under electron microscopy (EM) has a regular pattern of contractile proteins. Cross-sectional images of skeletal muscle cells have a regular hexagonal arrangement of six actin filaments surrounding every myosin filament. The structural organization of smooth muscle, on the other hand, is far more uncertain. Cross-sectional electron micrographs of smooth muscle indicate the presence of numerous filaments that, as of yet, seem to have no particular pattern (Fig.1.1) 


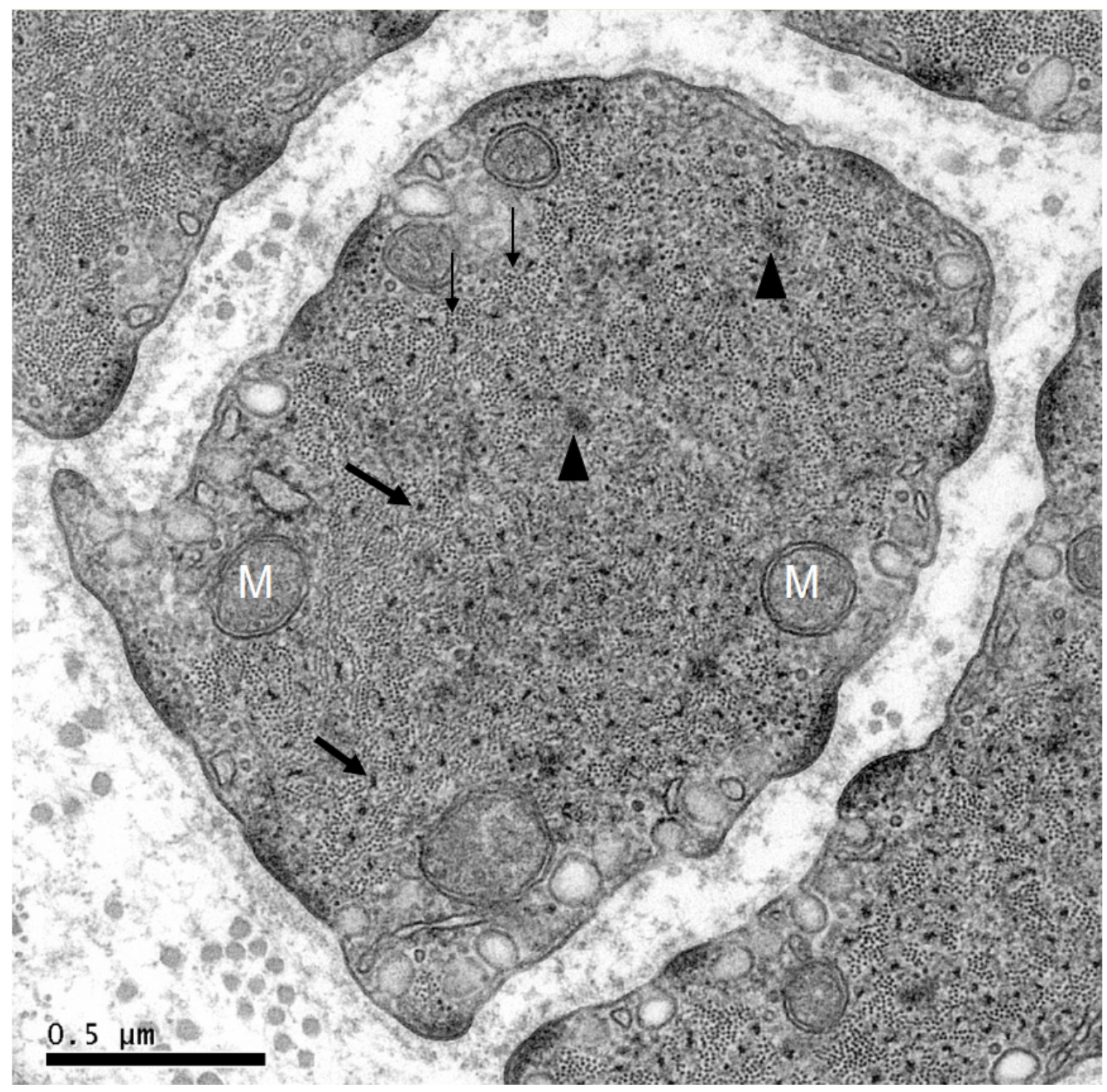

Figure 1.1 Transverse electron micrograph of a relaxed airway smooth muscle cell.

Large arrows indicate myosin thick filaments, small airways indicate actin thin filaments, ' $\mathrm{M}$ ' indicates mitochondria, and arrowheads indicate dense bodies. Bar $=0.5 \mu \mathrm{m}$.

Despite the lack of clarity in ultrastructure, the current model of the smooth muscle contractile unit is thought to be composed of at least two actin filaments flanking a sidepolar myosin filament $[7,8]$. This model has been supported by various structural studies 
$[3,9]$. Although the structure of the myosin filament in smooth muscle is more or less established, there is still much to be determined about the organization of the forcegenerating structures. Also, the compartmentalization of the cell into contractile and cytoskeletal domains has been suggested previously $[10,11]$, based on a similar paradigm in striated muscle, however, there is still much uncertainty as to the categorization of these components.

The traditional view has held that the actin filaments are attached to dense bodies which act as Z-disk equivalents in smooth muscle $[12,13]$. The Z-disk, which acts as an anchoring site for actin filaments in skeletal muscle, is composed of actin-binding proteins such as $\alpha$-actinin [13]. Likewise, dense bodies, which were first identified due to their dark electron-dense staining quality under EM, have been found to contain $\alpha$-actinin [13]. This comparison is not without some support. Actin filaments attach to Z-disks with their barbed $(+)$ ends, and likewise, actin has a similar polarity arrangement with dense bodies [14].

However, this traditional view has been called into question by Popescu and Ionescu [15] who questioned the equivalency between Z-disks and dense bodies. They found that treating skeletal muscle cells with $3 \mathrm{M}$ urea lead to full dissolution of the Z-disks but not the dense bodies in smooth muscle. Conversely, treatment of both cell types with WeberEdsall solution led to a removal of dense bodies, as examined under EM, but no change to Z-disks. This chemical inequality led them to conclude that dense bodies were not equivalent to Z-disks. Further, immunogold labeling of $\beta$-actin suggests that the dense 
bodies are not a part of the contractile apparatus [10] and may be compartmentalized separately of the contractile machinery (myosin and $\alpha$-actin). Since myosin filaments run parallel to the dense bodies and seem to be organized as long cable-like structures [16], not discrete units, their function in smooth muscle contraction has come into question. Furthermore, this baffling organization leads to uncertainty as to how the actin filaments are tethered to produce force.

\subsection{Myosin Evanescence and Mechanical Plasticity}

In the 1950s and 60s, there was great debate over whether smooth muscle cells contained myosin filaments at all [17]. While these initial studies of smooth muscle cells under EM were ambiguous, with some studies demonstrating the presence of myosin filaments, Xray diffraction confirmed the presence of filamentous myosin. Over time EM fixation techniques became more refined and myosin filaments were indeed found to be present in relaxed smooth muscle cells [17]. Regardless, these early difficulties demonstrated that smooth muscle myosin filaments were less robust than their counterparts in skeletal muscle. These early visualization issues masked the ability of smooth muscle myosin to assemble and disassemble filaments during a contraction-relaxation cycle [18-20].

The process of reforming and dissolving myosin filaments, termed myosin evanescence, may be an important contributor to the mechanical plasticity seen in smooth muscle cells. This plasticity allows the cell to undergo remarkable adaptation suggesting that the internal organization is capable of dynamic rearrangement. Recent evidence has supported a model in which the components of the contractile apparatus and cytoskeleton 
are malleable $[3,21-25]$. Besides the observation that contracted smooth muscle cells have more actin and myosin filaments than unstimulated cells [21,26], plasticity can be demonstrated by applying a length oscillation to a strip of smooth muscle (Fig. 1.2).

Prior to oscillation the muscle is held at an isometric length where it generates maximal force for that reference length (termed $F_{\max }$ ). After an oscillatory strain is applied, the amount of isometric force that the muscle can produce at the same reference length is reduced to $\sim 80 \% \mathrm{~F}_{\max }[27]$. If the ASM strip is maintained at that same length force will recover to $F_{\max }$ over a period of $\sim 30$ min. Immediately post-oscillation, the myosin filament density (number of filaments $/ \mu \mathrm{m}^{2}$ ) decreases to $\sim 80 \%$ of the initial density and gradually increases over $\sim 30 \mathrm{~min}$, following the same time course of force recovery [27]. These data suggest that myosin filaments are able to rapidly dissolve and assemble under different circumstances. However, the mechanisms guiding this assembly remain unknown.

Although Kuo et al. [27] did not measure other components of the cell, there is substantial evidence that thick filament assembly is not the only dynamic process that the cell undergoes during contraction. For instance, polymerization of actin filaments have been shown to occur with contraction as well as alterations in their adhesion to membrane tethering sites $[21,23,24]$. 


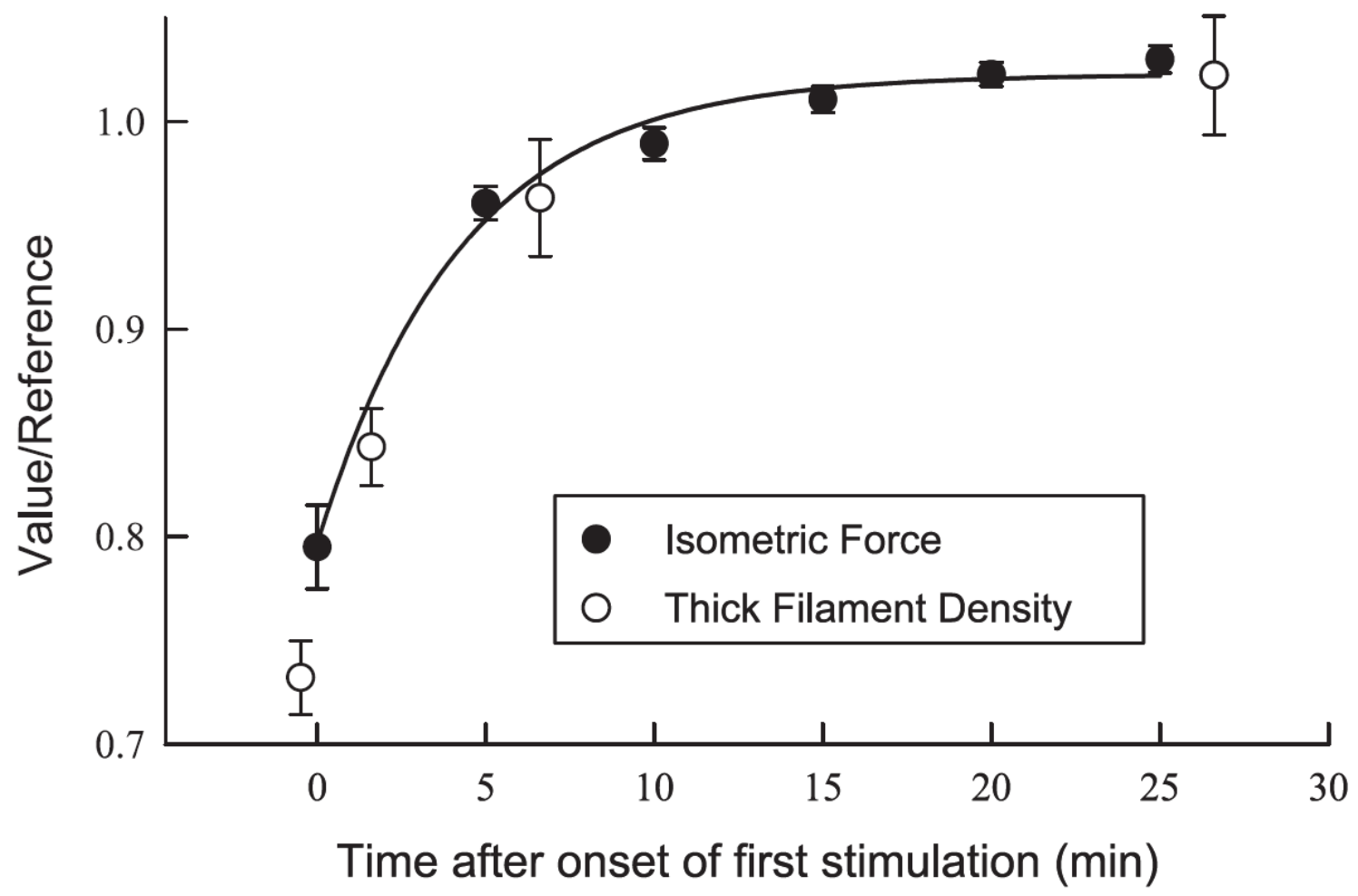

Figure 1.2 Recovery of force and thick filament density following length oscillation.

Prior to length oscillation the muscle was producing 1.0 force. After oscillation force declined and followed an exponential rate of recovery. The recovery of force coincided with an observed increase in thick filament density. Reproduced with permission from Kuo et al. [27].

\subsection{Smooth Muscle Signal Transduction}

Most contractile agonists stimulate smooth muscle through G-protein coupled receptors; such is the case with acetylcholine (ACh) stimulation. This activation leads to the production of inositol triphosphate $\left(\mathrm{IP}_{3}\right)$ and diacylglycerol (DAG) from phosphatidylinositol 4,5-bisphosphate $\left(\mathrm{PIP}_{2}\right)$ via phospholipase $\mathrm{C}$ cleavage. $\mathrm{IP}_{3}$ binds to its receptor on the sarcoendoplasmic reticulum (SR) leading to the release of intracellular 
calcium. The release of $\mathrm{Ca}^{2+}$ induces further $\mathrm{Ca}^{2+}$ release, a process termed calciuminduced calcium-release (CICR) from the ryanodine receptor (RyR) [28]. Mounting evidence also supports a mechanism in which $\mathrm{CD} 38$, also known as cyclic ADP ribose hydrolase, catalyzes the synthesis of cyclic ADP-ribose (cADPR) from $\mathrm{NAD}^{+}$and $\mathrm{ADP}^{-}$ ribose on the outer cell membrane [29]. cADPR is thought to then activate RyR release of $\mathrm{Ca}^{2+}$.

Once $\mathrm{Ca}^{2+}$ has been mobilized from the SR, four $\mathrm{Ca}^{2+}$ ions bind to calmodulin altering its conformation which, after complexing with myosin light chain kinase (MLCK), increases enzymatic activity. MLCK hydrolyzes ATP to ADP and phosphorylates the regulatory myosin light chain (rMLC) on the myosin molecule. This phosphorylation occurs at Ser19, but also may occur at Thr-18 [30], and is thought to increase the hydrolytic activity of the myosin- $\mathrm{Mg}^{2+}$-ATPase, thus regulating contraction [31].

Relaxation of the cell occurs by dephosphorylation of the rMLC by myosin light chain phosphatase (MLCP) and removal of $\mathrm{Ca}^{2+}$ through active transport across the cell membrane and sequestration into the SR via the sarcoendoplasmic reticulum $\mathrm{Ca}^{2+}$ ATPase (SERCA). While this signaling cascade of contraction-relaxation is well established, the role of peripheral signaling events is not well understood. However, it is clear that these signaling events are crucial to contraction. For instance, Rho and Rhokinase (ROCK) activation are involved in actin filamentogenesis [32] as well as calcium sensitization of contraction, in which MLCP activity is reduced, thus modulating contraction independent of intracellular concentration of $\mathrm{Ca}^{2+}$. Downstream effectors of 
DAG may also be important modulators of contraction. The role of these peripheral pathways is discussed further in Chapter Three.

\subsection{Myosin Filament Assembly}

Since the contraction-relaxation cycle is characterized by differential signaling pathways and a parallel formation and dissolution of myosin filaments, it is likely that differential protein phosphorylation, and not slower processes such as protein translation, signal transduction regulate myosin filamentogenesis. While it has long been known that in vitro formation of myosin filaments is affected by $\mathrm{MgATP}$ concentration, ionic strength, and $\mathrm{pH}$, phosphorylation of the rMLC is an important determinant of filament integrity $[33,34]$. Myosin monomers that are unphosphorylated at the 20 kDa-rMLC have been shown to fold such that the tail of the myosin monomer folds over the heads. Upon phosphorylation the tail elongates opening up the molecule, allowing the monomers to form a filament $[35,36]$. Additionally, phosphorylation of the rMLC has been shown to increase the stability of the myosin filament [33, 34]. While these observations provide evidence that $\mathrm{rMLC}$ regulation is an important factor in myosin filamentogenesis, it is unclear whether the rMLC signal is the cause of filament formation or a downstream signal. This idea is further elaborated in Chapter Three.

At physiological levels of MgATP, $\mathrm{pH}$, and ionic strength, myosin filaments can be dissolved in vitro [37]. This suggests that some additional factor(s) help(s) maintain myosin filament integrity in relaxed tissue (Fig. 1.1), where rMLC phosphorylation is at a

minimum. A possible candidate protein is the kinase-related protein, telokin. Telokin 
which is identical to the C-terminus of MLCK may also play a role in stabilizing unphosphorylated myosin filaments [38], by acting as a chaperone protein, with a stabilizing effect similar to that of rMLC.

The process of myosin filamentogenesis during the contraction-relaxation cycle suggests that before contraction there is an intracellular pool of myosin monomers that, upon stimulation of the muscle are incorporated into filaments (see Fig. 2.1) [37]. Presumably, the formation of myosin filaments requires guidance in order for the proper placement of filaments to produce force. Study of the assembly of thick filaments in Dictyostelium, an amoeboid slime mold, have demonstrated that filament formation is accelerated by the presence of actin filaments in vitro [39, 40]. Coupled with the fact that thick filaments must be formed within a thin filament lattice to produce force and are in great abundance in the relaxed cell (Fig. 1.1), it is possible that the actin filaments act as a 'scaffold' providing a site for filament formation. This guidance is probably directed by actinassociated proteins which act to stabilize thick filaments [37], such as caldesmon or calponin, proteins that bind both myosin and actin. This hypothesis was the primary objective of the study presented in Chapter Two. These unanswered questions can provide valuable information about smooth muscle contraction, and may be of great importance to the understanding of contraction in the context of asthma.

\subsection{Airway Smooth Muscle Function}

Smooth muscle cells line the airways throughout the entire bronchial tree. In the trachea, parallel bundles of trachealis muscle are attached to the C-shaped cartilage rings. The 
muscle, found on the posterior side of the trachea, is an ideal source for ASM because of the neat arrangement of parallel bundles, perpendicular to the long axis of the trachea. Further down the bronchial tree, the ASM encircles the airways in a helical fashion which suggests that contraction would both narrow the airways and shorten their length [41, 42]. While ASM is responsible for the exaggerated airway narrowing characteristic of asthma, its physiological function is the subject of much debate.

There has been much speculation about the possible physiological function of ASM. Some have argued that ASM contraction is responsible for stiffening the airways, and thus preventing airway collapse during periods of negative transmural pressures such as during forced expiration [43]. Others have suggested that the ASM acts to optimize ventilation by adjusting airway caliber, or improves the effectiveness of coughing [44]. While Mead [45] has suggested a role for ASM in controlling mucus clearance, Ford [46] has argued that ASM contraction acts as a life-saving warning system during partial airway closure, by alerting an individual to a harmful substance. Contrarily, Mitzner [4750] and others have argued that ASM is the 'appendix' of the lung, a function-less tissue that is present due to its embryological origins. Bronchial thermoplasty, a procedure in which the ASM (and associated tissues of the airways) is ablated by heating the tissue with radiofrequency energy $[47,51]$, supports this view since patients and animals treated with thermoplasty have shown no adverse side effects. 


\subsection{Asthma and Airway Smooth Muscle}

Asthma is a chronic inflammatory disease characterized by recurring symptoms of cough, wheezing, and shortness of breath [52]. It is often associated with airway inflammatory cell infiltration and over time, remodeling of the airways. The heterogeneous nature of the disease complicates investigations into its etiology. While it is frequently associated with allergy and atopy, other factors, such as obesity and dry, cold air (exercise-induced asthma) have been identified as causal agents. The 'twitchy' airways of asthmatics have an increased reactivity and sensitivity to a wide variety of stimuli, such as histamine or leukotrienes [53]. While this airway hyperresponsiveness (AHR) is apparent in asthmatic patients and clinically determined through airway flow measurements (e.g. forced expiratory volume in the first second of a forced expiratory maneuver, $\mathrm{FEV}_{1}$ ), it is unclear whether AHR is a property of ASM function or a property of the non-contractile components of the airway [54].

The airways of asthmatics are known to have a greater proportion of ASM, however, whether this is due to hypertrophy or hyperplasia of the smooth muscle layer is unknown [55]. Mathematical modeling of the airways suggests that increased ASM mass without changes to its contractile phenotype, would, in itself, be a significant contributor to exaggerated airway narrowing by allowing greater shortening [56]. Of course, asthma is also associated with airway remodeling which through edema, hyperemia, extracellular matrix thickening, and adventitial thickening, could result in AHR independent of changes in ASM function. A plausible hypothesis would be to examine whether asthmatic ASM in vitro demonstrates increased sensitivity and responsiveness to stimuli. 
Unfortunately, data on asthmatic ASM has been limited and the results inconclusive. This is due, in part, to the fact that human ASM has been difficult to obtain, thus, asthmatic ASM research often relies on animal models of disease. The study presented in Chapter Four provides a valuable comparison between non-asthmatic human ASM and animal ASM tissues.

While increased reactivity of ASM (i.e. force generating capacity) has only been adequately determined in two previous studies [57, 58], by normalizing force produced by the cross-sectional area of the muscle, increased sensitivity to various stimuli has been extensively investigated. Asthmatic ASM was found to be more responsive than nonasthmatic ASM to similar concentrations of various contractile agonists (leukotriene C4, acetylcholine, methacholine, histamine, and electrical field stimulation) in three studies $[57,59,60]$, but no difference was found in another nine studies [58, 61-68]. The studies that demonstrated a difference were limited, with two of them [57, 59] performed on only one asthmatic subject.

Another defining property of asthmatic airways is the reduced bronchodilating effect of deep inspirations (DI). During normal tidal breathing airways have a tendency to narrow. Intermittent DIs expand the airways in non-asthmatics and help maintain airway patency. In fact, DIs are thought to be most potent bronchodilator even compared to pharmaceuticals $[69,70]$. In asthmatics, this dilatory effect is lost. Some have suggested that this is due to an uncoupling of the airways and parenchyma at the alveolar attachment sites [71]. Mauad et al. [71] demonstrated that asthmatic airways have 
decreased elastic fiber content at the alveolar attachment sites compared to nonasthmatics, which would lead to decreased elastic recoil and reduce the bronchodilating effect. This effect could also be manifested by an increased stiffness in asthmatic ASM [72] (see Chapter 5) or a differential responsiveness by asthmatic ASM to mechanical perturbation compared with non-asthmatic ASM. Since the dilating of the airways leads to stretching of the ASM, this effect can be mimicked in vitro by mechanically perturbing the ASM. Studies of non-diseased animal ASM have shown that mechanical perturbation reduces force generating capacity - both length and force oscillations [73-75]. Whether asthmatic ASM responds differently to mechanical perturbation than non-asthmatic ASM has not been previously examined. The study presented in Chapter Five provides valuable data on the responsiveness of asthmatic ASM to mechanical perturbation and compares the mechanical properties of asthmatic ASM with non-asthmatic ASM.

\subsection{Experimental Summary}

In the following chapters, the mechanical properties of airway smooth muscle in health and disease will be examined. The first two studies will investigate the contractile mechanisms responsible for force generation in healthy ASM. Chapter Two focuses on determining whether the actin filament lattice supports myosin filaments in relaxed smooth muscle cells. To test this hypothesis the muscle was treated with a known actin filament depolymerizing agent, cytochalasin $\mathrm{D}$, in order to determine whether removal of the lattice 'scaffold' would lead to a reduction in myosin filaments. Chapter Three focuses on the contractile responses to potassium chloride. When compared to acetylcholine-induced contraction, $\mathrm{KCl}$ caused a contraction of similar force but with far 
fewer myosin filaments. This disparity of myosin filamentogenesis during contraction between the two contractile agonists will be examined in detail. Chapter Four and Five focus on the mechanical properties of human ASM. The study in Chapter Four focused on describing the mechanical properties of non-asthmatic human ASM and comparing these properties to previous ASM mechanical studies in other mammalian species and previous human ASM studies. In Chapter Five these mechanical properties of nonasthmatic ASM were compared with the properties of asthmatic ASM. Both studies examined force generating capacity, force-length relationships, the ability to undergo length adaptation, shortening capacity and velocity, and responsiveness to mechanical perturbation. 


\subsection{References}

1. Huxley AF and Niedergerke R. Structural changes in muscle during contraction; interference microscopy of living muscle fibres. Nature 1954; 173: 4412: 971-973.

2. Huxley $\mathrm{H}$ and Hanson J. Changes in the cross-striations of muscle during contraction and stretch and their structural interpretation. Nature 1954; 173: 4412: 973-976.

3. Herrera AM, McParland BE, Bienkowska A, Tait R, Pare PD, Seow CY. 'Sarcomeres' of smooth muscle: functional characteristics and ultrastructural evidence. J.Cell.Sci. 2005; 118: Pt 11: 2381-2392.

4. An SS and Fredberg JJ. Biophysical basis for airway hyperresponsiveness. Can.J.Physiol.Pharmacol. 2007; 85: 7: 700-714.

5. Huxley AF. Muscle structure and theories of contraction. Prog.Biophys.Biophys.Chem. 1957; 7: 255-318.

6. Huxley AF and Niedergerke R. Measurement of muscle striations in stretch and contraction. J.Physiol. 1954; 124: 2: 46-7P.

7. Craig R and Megerman J. Assembly of smooth muscle myosin into side-polar filaments. J.Cell Biol. 1977; 75: 3: 990-996.

8. Craig R and Woodhead JL. Structure and function of myosin filaments. Curr.Opin.Struct.Biol. 2006; 16: 2: 204-212.

9. Xu JQ, Harder BA, Uman P, Craig R. Myosin filament structure in vertebrate smooth muscle. J.Cell Biol. 1996; 134: 1: 53-66.

10. North AJ, Gimona M, Lando Z, Small JV. Actin isoform compartments in chicken gizzard smooth muscle cells. J.Cell.Sci. 1994; 107 ( Pt 3): Pt 3: 445-455.

11. Small JV and Gimona M. The cytoskeleton of the vertebrate smooth muscle cell. Acta Physiol.Scand. 1998; 164: 4: 341-348.

12. Hanson J. The structure of the smooth muscle fibres in the body wall of the earth worm. J.Biophys.Biochem.Cytol. 1957; 3: 1: 111-122.

13. Geiger B, Dutton AH, Tokuyasu KT, Singer SJ. Immunoelectron microscope studies of membrane-microfilament interactions: distributions of alpha-actinin, tropomyosin, and vinculin in intestinal epithelial brush border and chicken gizzard smooth muscle cells. J.Cell Biol. 1981; 91: 3 Pt 1: 614-628. 
14. Bond M and Somlyo AV. Dense bodies and actin polarity in vertebrate smooth muscle. J.Cell Biol. 1982; 95: 2 Pt 1: 403-413.

15. Popescu LM and Ionescu N. On the equivalence between dense bodies and Z-bands. Experientia 1970; 26: 6: 642-643.

16. McGuffee LJ and Little SA. Three-dimensional characterization of dense bodies in contracted and relaxed mesenteric artery smooth muscle cells. Scanning Microsc. 1992; 6: 3: 837-845.

17. Somlyo AV, Ashton FT, Lemanski LF, Vallieres J and and Somlyo AP. Filament organization and dense bodies in vertebrate smooth muscle. In: , edited by Stephens NL. Baltimore, MD.: University Park Press, 1977, p. 445.

18. Smolensky AV, Ragozzino J, Gilbert SH, Seow CY, Ford LE. Length-dependent filament formation assessed from birefringence increases during activation of porcine tracheal muscle. J.Physiol. 2005; 563: Pt 2: 517-527.

19. Ford LE. Plasticity in airway smooth muscle: an update. Can.J.Physiol.Pharmacol. 2005; 83: 10: 841-850.

20. Herrera AM, Kuo KH, Seow CY. Influence of calcium on myosin thick filament formation in intact airway smooth muscle. Am.J.Physiol Cell Physiol 2002; 282: 2: C310-C316.

21. Herrera AM, Martinez EC, Seow CY. Electron microscopic study of actin polymerization in airway smooth muscle. Am.J.Physiol.Lung Cell.Mol.Physiol. 2004; 286: 6: L1161-8.

22. Gunst SJ, Meiss RA, Wu MF, Rowe M. Mechanisms for the mechanical plasticity of tracheal smooth muscle. Am.J.Physiol. 1995; 268: 5 Pt 1: C1267-76.

23. Opazo Saez A, Zhang W, Wu Y, Turner CE, Tang DD, Gunst SJ. Tension development during contractile stimulation of smooth muscle requires recruitment of paxillin and vinculin to the membrane. Am.J.Physiol.Cell.Physiol. 2004; 286: 2: C433-47.

24. Zhang W and Gunst SJ. Dynamic association between alpha-actinin and beta-integrin regulates contraction of canine tracheal smooth muscle. J.Physiol. 2006; 572: Pt 3: 659-676.

25. Pratusevich VR, Seow CY, Ford LE. Plasticity in canine airway smooth muscle. J.Gen.Physiol. 1995; 105: 1: 73-94. 
26. Mehta D and Gunst SJ. Actin polymerization stimulated by contractile activation regulates force development in canine tracheal smooth muscle. J.Physiol. 1999; 519 Pt 3: 829-840.

27. Kuo KH, Wang L, Pare PD, Ford LE, Seow CY. Myosin thick filament lability induced by mechanical strain in airway smooth muscle. J.Appl.Physiol. 2001; 90: 5: 1811-1816.

28. Itoh $\mathrm{T}$, Ueno $\mathrm{H}$, Kuriyama $\mathrm{H}$. Calcium-induced calcium release mechanism in vascular smooth muscles--assessments based on contractions evoked in intact and saponin-treated skinned muscles. Experientia 1985; 41: 8: 989-996.

29. Sieck GC, White TA, Thompson MA, Pabelick CM, Wylam ME, Prakash YS. Regulation of store-operated Ca2+ entry by CD38 in human airway smooth muscle. Am.J.Physiol.Lung Cell.Mol.Physiol. 2008; 294: 2: L378-85.

30. Feng J, Ito M, Nishikawa M, Okinaka T, Isaka N, Hartshorne DJ, Nakano T. Dephosphorylation of distinct sites on the $20 \mathrm{kDa}$ myosin light chain by smooth muscle myosin phosphatase. FEBS Lett. 1999; 448: 1: 101-104.

31. Sobieszek A and Small JV. Regulation of the actin-myosin interaction in vertebrate smooth muscle: activation via a myosin light-chain kinase and the effect of tropomyosin. J.Mol.Biol. 1977; 112: 4: 559-576.

32. Chaudhuri S and Smith PG. Cyclic strain-induced HSP27 phosphorylation modulates actin filaments in airway smooth muscle cells. Am.J.Respir.Cell Mol.Biol. 2008; 39: 3: $270-278$.

33. Ip K, Sobieszek A, Solomon D, Jiao Y, Pare PD, Seow CY. Physical integrity of smooth muscle myosin filaments is enhanced by phosphorylation of the regulatory myosin light chain. Cell.Physiol.Biochem. 2007; 20: 5: 649-658.

34. Suzuki H, Onishi H, Takahashi K, Watanabe S. Structure and function of chicken gizzard myosin. J.Biochem. 1978; 84: 6: 1529-1542.

35. Kendrick-Jones J, Smith RC, Craig R, Citi S. Polymerization of vertebrate nonmuscle and smooth muscle myosins. J.Mol.Biol. 1987; 198: 2: 241-252.

36. Trybus KM and Lowey S. Assembly of smooth muscle myosin minifilaments: effects of phosphorylation and nucleotide binding. J.Cell Biol. 1987; 105: 6 Pt 2: 3007-3019.

37. Seow CY. Myosin filament assembly in an ever-changing myofilament lattice of smooth muscle. Am.J.Physiol.Cell.Physiol. 2005; 289: 6: C1363-8. 
38. Nieznanski $\mathrm{K}$ and Sobieszek A. Telokin (kinase-related protein) modulates the oligomeric state of smooth-muscle myosin light-chain kinase and its interaction with myosin filaments. Biochem.J. 1997; 322 ( Pt 1): Pt 1: 65-71.

39. Mahajan RK, Vaughan KT, Johns JA, Pardee JD. Actin filaments mediate Dictyostelium myosin assembly in vitro. Proc.Natl.Acad.Sci.U.S.A. 1989; 86: 16: 6161-6165.

40. Mahajan RK and Pardee JD. Assembly mechanism of Dictyostelium myosin II: regulation by $\mathrm{K}+, \mathrm{Mg} 2+$, and actin filaments. Biochemistry 1996; 35: 48: 1550415514.

41. Amrani Y, Chen H, Panettieri RA,Jr. Activation of tumor necrosis factor receptor 1 in airway smooth muscle: a potential pathway that modulates bronchial hyperresponsiveness in asthma? Respir.Res. 2000; 1: 1: 49-53.

42. Stephens NL and Halayko AJ. Airway smooth muscle contractile, regulatory and cytoskeletal protein expression in health and disease. Comp.Biochem.Physiol.B.Biochem.Mol.Biol. 1998; 119: 3: 415-424.

43. Seow CY, Wang L, Pare PD. Airway narrowing and internal structural constraints. J.Appl.Physiol. 2000; 88: 2: 527-533.

44. Seow CY and Fredberg JJ. Historical perspective on airway smooth muscle: the saga of a frustrated cell. J.Appl.Physiol. 2001; 91: 2: 938-952.

45. Mead J. Point: airway smooth muscle is useful. J.Appl.Physiol. 2007; 102: 4: 1708-9; discussion 1710 .

46. Ford LE. Comment on point:counterpoint: "airway smooth muscle is/is not useful". J.Appl.Physiol. 2007; 102: 6: 2407.

47. Mitzner W. Bronchial thermoplasty in asthma. Allergol.Int. 2006; 55: 3: 225-234.

48. Mitzner W. Comments on point: counterpoint: "airway smooth muscle is/is not useful". J.Appl.Physiol. 2007; 102: 4: 1712; author reply 1723.

49. Mitzner W. A further comment on Point:Counterpoint "Airway smooth muscle is/is not useful". J.Appl.Physiol. 2008; 104: 3: 902.

50. Mitzner W. Airway smooth muscle: the appendix of the lung. Am.J.Respir.Crit.Care Med. 2004; 169: 7: 787-790.

51. Cox G, Miller JD, McWilliams A, Fitzgerald JM, Lam S. Bronchial thermoplasty for asthma. Am.J.Respir.Crit.Care Med. 2006; 173: 9: 965-969. 
52. Guerra S, Wright AL, Morgan WJ, Sherrill DL, Holberg CJ, Martinez FD. Persistence of asthma symptoms during adolescence: role of obesity and age at the onset of puberty. Am.J.Respir.Crit.Care Med. 2004; 170: 1: 78-85.

53. Hargreave FE, O'Byrne PM, Ramsdale EH. Mediators, airway responsiveness, and asthma. J.Allergy Clin.Immunol. 1985; 76: 2 Pt 2: 272-276.

54. McParland BE, Tait RR, Pare PD, Seow CY. The role of airway smooth muscle during an attack of asthma simulated in vitro. Am.J.Respir.Cell Mol.Biol. 2005; 33: 5: $500-504$.

55. Ebina M, Takahashi T, Chiba T, Motomiya M. Cellular hypertrophy and hyperplasia of airway smooth muscles underlying bronchial asthma. A 3-D morphometric study. Am.Rev.Respir.Dis. 1993; 148: 3: 720-726.

56. Lambert RK, Wiggs BR, Kuwano K, Hogg JC, Pare PD. Functional significance of increased airway smooth muscle in asthma and COPD. J.Appl.Physiol. 1993; 74: 6: 2771-2781.

57. Bramley AM, Thomson RJ, Roberts CR, Schellenberg RR. Hypothesis: excessive bronchoconstriction in asthma is due to decreased airway elastance. Eur.Respir.J. 1994; $7: 2: 337-341$.

58. Thomson RJ, Bramley AM, Schellenberg RR. Airway muscle stereology: implications for increased shortening in asthma. Am.J.Respir.Crit.Care Med. 1996; 154: 3 Pt 1: 749-757.

59. de Jongste JC, Mons H, Bonta IL, Kerrebijn KF. In vitro responses of airways from an asthmatic patient. Eur.J.Respir.Dis. 1987; 71: 1: 23-29.

60. Bai TR. Abnormalities in airway smooth muscle in fatal asthma. Am.Rev.Respir.Dis. 1990; 141: 3: 552-557.

61. Bai TR. Abnormalities in airway smooth muscle in fatal asthma. A comparison between trachea and bronchus. Am.Rev.Respir.Dis. 1991; 143: 2: 441-443.

62. Goldie RG, Spina D, Henry PJ, Lulich KM, Paterson JW. In vitro responsiveness of human asthmatic bronchus to carbachol, histamine, beta-adrenoceptor agonists and theophylline. Br.J.Clin.Pharmacol. 1986; 22: 6: 669-676.

63. Whicker SD, Armour CL, Black JL. Responsiveness of bronchial smooth muscle from asthmatic patients to relaxant and contractile agonists. Pulm.Pharmacol. 1988; 1: $1: 25-31$. 
64. Cerrina J, Le Roy Ladurie M, Labat C, Raffestin B, Bayol A, Brink C. Comparison of human bronchial muscle responses to histamine in vivo with histamine and isoproterenol agonists in vitro. Am.Rev.Respir.Dis. 1986; 134: 1: 57-61.

65. Cerrina J, Labat C, Haye-Legrande I, Raffestin B, Benveniste J, Brink C. Human isolated bronchial muscle preparations from asthmatic patients: effects of indomethacin and contractile agonists. Prostaglandins 1989; 37: 4: 457-469.

66. Schellenberg RR and Foster A. In vitro responses of human asthmatic airway and pulmonary vascular smooth muscle. Int.Arch.Allergy Appl.Immunol. 1984; 75: 3: 237-241.

67. Roberts JA, Raeburn D, Rodger IW, Thomson NC. Comparison of in vivo airway responsiveness and in vitro smooth muscle sensitivity to methacholine in man. Thorax 1984; 39: 11: 837-843.

68. Bjorck T, Gustafsson LE, Dahlen SE. Isolated bronchi from asthmatics are hyperresponsive to adenosine, which apparently acts indirectly by liberation of leukotrienes and histamine. Am.Rev.Respir.Dis. 1992; 145: 5: 1087-1091.

69. Nadel JA and Tierney DF. Effect of a previous deep inspiration on airway resistance in man. J.Appl.Physiol. 1961; 16: 717-719.

70. Gump A, Haughney L, Fredberg J. Relaxation of activated airway smooth muscle: relative potency of isoproterenol vs. tidal stretch. J.Appl.Physiol. 2001; 90: 6: 23062310.

71. Mauad T, Silva LF, Santos MA, Grinberg L, Bernardi FD, Martins MA, Saldiva PH, Dolhnikoff M. Abnormal alveolar attachments with decreased elastic fiber content in distal lung in fatal asthma. Am.J.Respir.Crit.Care Med. 2004; 170: 8: 857-862.

72. Bramley AM, Roberts CR, Schellenberg RR. Collagenase increases shortening of human bronchial smooth muscle in vitro. Am.J.Respir.Crit.Care Med. 1995; 152: $5 \mathrm{Pt}$ 1: $1513-1517$.

73. Wang L, Pare PD, Seow CY. Effects of length oscillation on the subsequent force development in swine tracheal smooth muscle. J.Appl.Physiol. 2000; 88: 6: 22462250 .

74. Fredberg JJ, Inouye D, Miller B, Nathan M, Jafari S, Raboudi SH, Butler JP, Shore SA. Airway smooth muscle, tidal stretches, and dynamically determined contractile states. Am.J.Respir.Crit.Care Med. 1997; 156: 6: 1752-1759.

75. Fredberg JJ, Inouye DS, Mijailovich SM, Butler JP. Perturbed equilibrium of myosin binding in airway smooth muscle and its implications in bronchospasm. Am.J.Respir.Crit.Care Med. 1999; 159: 3: 959-967. 


\section{CHAPTER 2. FACILITATION OF MYOSIN FILAMENTOGENESIS BY THE ACTIN FILAMENT LATTICE ${ }^{1}$}

\subsection{Introduction}

Airway smooth muscle (ASM) is responsible for the excessive airway narrowing characteristic of asthma. The cause of this excessive narrowing is unknown. However, it has been suggested that it could be due to increased ASM mass in the airways or to intrinsic alterations within the ASM cells that allow them to produce more force and shorten to a greater extent in asthmatic patients. Regardless of the cause, it has become clear that our understanding of the pathophysiology of asthma relates to the ability of ASM to generate force. Vital to this aim is an understanding of how ASM cells organize their contractile filaments and the mechanisms which govern this organization.

Smooth muscle cells are present in many organ systems and often line hollow organs. Through contraction these smooth muscle cells, for instance, in the urinary bladder or blood vessels, control organ volume and thus maintain homeostasis. While the physiological function of ASM is not well understood (see Chapter One), the muscle has a similar volume regulating capability (i.e. the volume and caliber of the conducting airways). The vast volume changes which occur in some hollow organs requires the smooth muscle to operate over an extreme length range compared to striated muscle which is restricted by their origins and insertions. In order for smooth muscle cells to operate across these large length ranges, the cells require a dynamic contractile apparatus.

\footnotetext{
${ }^{1}$ A version of this chapter will be submitted for publication. Chin LYM, Ip K, Solomon D, Paré PD, Seow CY. (2010) Facilitation of myosin filamentogenesis by the actin filament lattice.
} 
Smooth muscle cells, unlike striated muscle cells, are capable of generating near maximal force over a broad length range through a process known as length adaptation [1]. Initially following a length change all muscle cells have a reduced force capacity, this is due to a decreased overlap between actin thin filaments and myosin thick filaments. Given a certain period of time, smooth muscle cells are capable of adapting to a given length and generate nearly maximal force [1]. This type of adaptation would require months of intracellular reorganization in striated muscle, but occurs in a few minutes in smooth muscle. This ability, known as mechanical plasticity, requires the cells to be labile and capable of rapid internal rearrangement [2]. Indeed, a myriad of changes has been shown to occur during the relaxation-contraction cycle. Actin and myosin filamentogenesis as well as focal adhesion complexing have been shown to occur during contraction [3-5].

In the relaxed state it is unclear how myosin filaments are stabilized (as in e.g. Fig. 1.1), given that at physiological concentrations of $\mathrm{Mg}^{2+}-\mathrm{ATP}, \mathrm{pH}$, and ionic strength the filaments have been shown to disassemble in vitro $[2,6]$. Phosphorylation of the regulatory myosin light chain (rMLC) has been shown to stabilize filaments $[6,7]$ and promote myosin filamentogenesis $[8,9]$, in addition to its role in regulating acto-myosin crossbridge cycling [10]. Since relaxed smooth muscle cells have low levels of rMLC (see Fig. 3.11), the presence of myosin filaments is likely supported by stabilizing proteins [2]. During activation, rMLC is phosphorylated and there is rapid increase in myosin filaments, however, the mechanism of this myosin evanescence, is unknown (see Fig. 3.4 and 3.5). One hypothesis, outlined in Fig. 2.1, suggests that myosin filaments are 
rapidly assembled during contraction through a facilitative ability of the actin filament lattice. Given that actin filaments greatly outnumber myosin filaments $(\sim 30: 1$ in relaxed cells), it is plausible that the actin filaments act as a 'scaffold', guiding myosin filamentogenesis (Fig. 2.1) [2].

The purpose of this study was to determine whether the actin filament lattice supports myosin filaments in relaxed smooth muscle cells. This hypothesis has some support from studies of Dictyostelium thick filament assembly, in which myosin filament formation was found to accelerate in the presence of actin filaments [11, 12]. To test this hypothesis muscle strips were treated with a fungal metabolite known to disrupt actin filaments. Actin occurs in cells in globular form (G-actin), as a 42-kDa protein, and in a filamentous form (F-actin) with a filament diameter of 6-8 $\mathrm{nm}$. The process of forming actin filaments is under strict regulation of various actin binding proteins. In a filament, the molecules have a polarity in which the barbed or $(+)$ end, undergoes rapid monomer addition, whereas the pointed or (-) end undergoes gradual monomer removal [13]. This is a dynamic process, referred to as treadmilling, as continual monomer addition to one end and subtraction from the other, maintains a constant filament length. Thus, capping proteins, of either the (+) or (-) end are able to regulate filament length. Cytochalasin D (CD), also known as Zygosporin A, a metabolite of the fungus Zygosporium mansonii is known to bind to the barbed (+) end of actin filaments. By attaching to the lengthening barbed $(+)$ end, $\mathrm{CD}$ has been shown to shift the polymerization/depolymerization equilibrium towards actin filament shortening, in a variety of cell types [14]. 


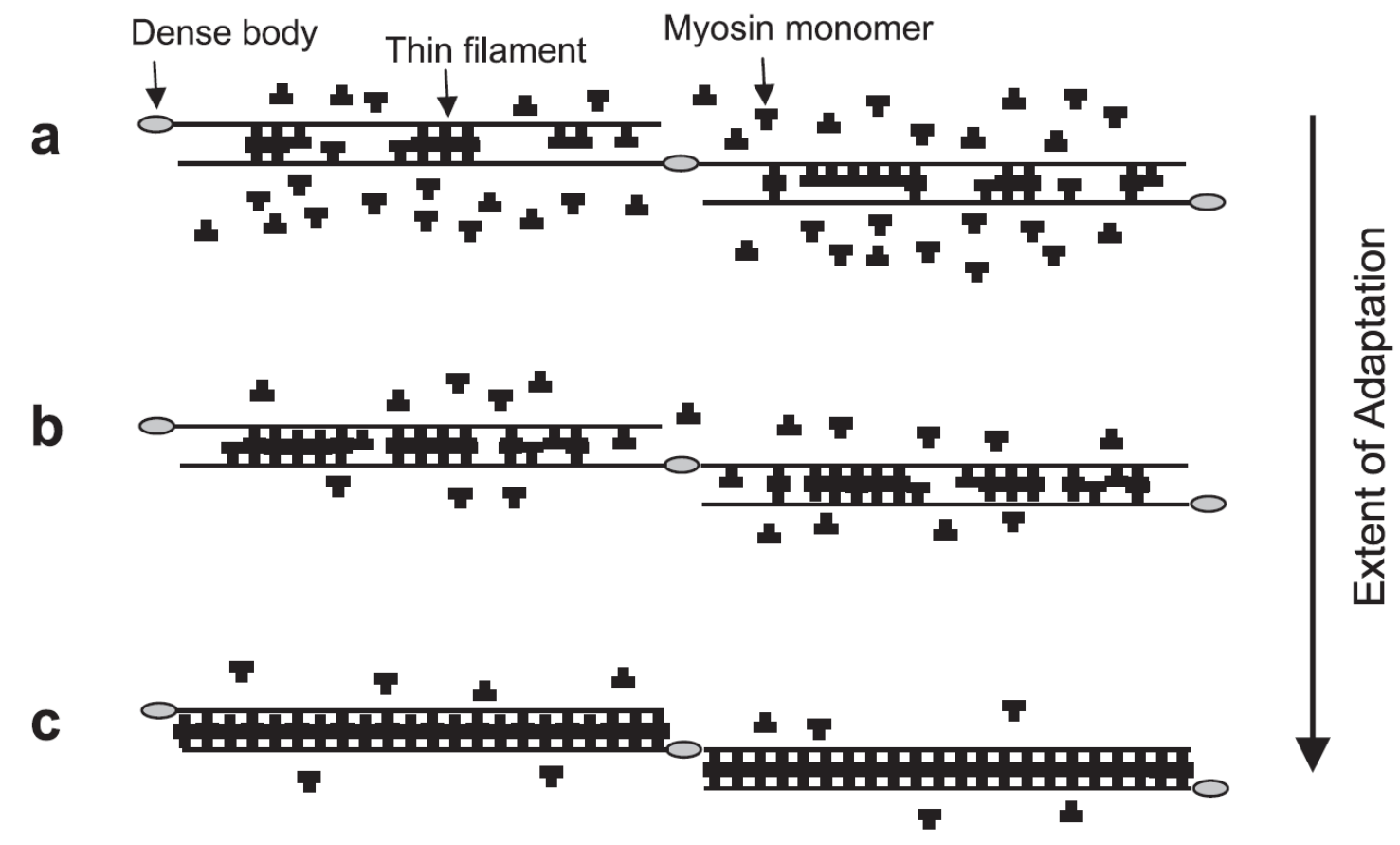

Figure 2.1 Hypothetical model of myosin filament formation facilitated by the actin

filament lattice. Following a depolymerizing event, such as a mechanical perturbation, the myosin filament in the contractile units has been fragmented (a). This could also represent de novo assembly of a myosin filament during activation. b) As the period of recovery or adaptation continues, myosin monomers are somehow guided into the contractile units (b), until a full filament is formed (c). This facilitation may be due to actin associated proteins, such as caldesmon. See text for more details. Reproduced with permission from Seow [2]. 


\subsection{Materials and Methods}

\subsubsection{Tissue Preparation and Equilibration}

Sheep tracheas were obtained from a local abattoir and prepared as previously described [15]. All experimental procedures were approved by the Animal Care Committee and Biosafety Committee of the University of British Columbia and conformed to the guidelines set out by the Canadian Council on Animal Care (Appendix A). Briefly, tracheas were stored in $4^{\circ} \mathrm{C}$ physiological saline solution (PSS) upon arrival at the University. The PSS consisted of (mM): $118 \mathrm{NaCl}, 5 \mathrm{KCl}, 1.2 \mathrm{NaH}_{2} \mathrm{PO}_{4}, 22.5 \mathrm{NaHCO}_{3}$, $2 \mathrm{MgSO}_{4}, 2 \mathrm{CaCl}_{2}$, and $2 \mathrm{~g} / \mathrm{L}$ dextrose. Experiments were performed within five days of obtaining the tissue. The in situ parallel arrangement of smooth muscle bundles in the trachea and low resting tension of the muscle, make it an ideal site for muscle strip extraction. In situ muscle length was used as a reference length $\left(\mathrm{L}_{\text {ref }}\right)$ for normalization of length measurements, and was determined from a 3/4" tracheal segment prior to removal of the C-shaped cartilage. After removal of the cartilage, the muscle bundles, held at in situ length, were dissected free of adventitial connective tissue and epithelium. Muscle strips measuring 1-1.5 mm wide, 0.3-0.5 mm thick, and $6 \mathrm{~mm}$ in length were removed and fastened with aluminum foil clips at each end. The muscle strips were then vertically mounted onto a custom-designed force-length transducer myograph apparatus. While on the myograph, the muscle was entirely submerged in a tissue bath filled with warmed PSS $\left(37^{\circ} \mathrm{C}\right)$. An outer water-jacket insulated the tissue bath from heat loss with continually circulating $37^{\circ} \mathrm{C}$ water. 
The muscle strips held at $\mathrm{L}_{\text {ref }}$ were initially stimulated for a duration of 10 seconds with electrical field stimulation (EFS) at 5 minute intervals during a $1.5 \mathrm{~h}$ period of equilibration. EFS consisted of a $60 \mathrm{~Hz}$ alternating current at a voltage of $15-20 \mathrm{~V}$ which resulted in maximal force generation. PSS gassed with $5 \% \mathrm{CO}_{2} / 95 \% \mathrm{O}_{2}$ to maintain a $\mathrm{pH}$ of 7.4 was refreshed in the tissue bath at 5 minute intervals following contraction. The tissue was considered equilibrated when the muscle generated consistent maximal isometric force $\left(\mathrm{F}_{\max }\right)$ with minimal resting tension.

\subsubsection{Protocol 1: Treatment with $1 \mu M$ Cytochalasin D}

After equilibration the muscle was treated with PSS containing $1 \mu$ M CD (Sigma C8273: Sigma-Aldrich). CD was dissolved into a delivery vehicle of $0.1 \%$ DMSO. Paired muscle strips, obtained from the same trachea were incubated with either $1 \mu \mathrm{M} \mathrm{CD}$ or $0.1 \%$ DMSO, the vehicle control (VC), for $1 \mathrm{~h}$. The muscle was stimulated with EFS at 5 minute intervals during this hour and the PSS replenished after each contraction. Following this incubation period, the muscle strips were fixed for electron microscopy as described below.

\subsubsection{Protocol 2: Treatment with $5 \mu \mathrm{M}$ Cytochalasin D}

In a separate set of experiments, paired muscle strips were treated with PSS containing the $0.1 \%$ DMSO vehicle or $5 \mu \mathrm{MCD}$ immediately before a passive 10 minute length oscillation. The length oscillation consisted of a sinusoidal wave pattern with a frequency of $0.25 \mathrm{~Hz}$ and a stretch amplitude of $30 \% \mathrm{~L}_{\text {ref. }}$. Following oscillation the muscle strips were stimulated at 5 minute intervals for five contractions (Fig. 2.6). Afterwards, the muscle strips were fixed for electron microscopy as described below. 


\subsubsection{Electron Microscopy}

Following treatment with either the $\mathrm{VC}$ or $\mathrm{CD}$, the muscle strips were fixed in a relaxed state immediately after the last EFS contraction. The protocol for EM fixation has been described previously [5]. Briefly, the muscle was placed in a formaldehyde cocktail consisting of: $2 \%$ paraformaldehyde, $1 \%$ glutaraldehyde, and $2 \%$ tannic acid in $0.1 \mathrm{M}$ sodium cacodylate solution warmed to $37^{\circ} \mathrm{C}$. After initial fixation the muscle was cut into 6-8 blocks and incubated in the formaldehyde cocktail on a shaker at $4^{\circ} \mathrm{C}$ for $2 \mathrm{~h}$. The blocks were then washed thrice with $0.1 \mathrm{M}$ sodium cacodylate. The tissue blocks underwent a secondary fixation with $1 \% \mathrm{OsO}_{4}-0.1 \mathrm{M}$ sodium cacodylate for $2 \mathrm{~h}$, followed by three washes in distilled water (10 min each). En bloc staining was accomplished by placing the blocks in $1 \%$ uranyl acetate for $1 \mathrm{~h}$, followed by three washes with distilled water, as above. The blocks were then dehydrated using increasing concentrations of ethanol $(50,70,80,90,95 \%$ - 10 min each; $100 \%$ - $3 \times 15$ min each) followed by three washes with propylene oxide (15 $\mathrm{min}$ each). The blocks were gradually infiltrated overnight with TAAB 812 resin mix and then embedded in molds and placed in an oven at $60^{\circ} \mathrm{C}$ overnight. The embedded blocks were sectioned on a microtome with a diamond knife and placed on copper mesh grids. The section thickness was $50 \mathrm{~nm}$. After sectioning the grids were stained with $1 \%$ uranyl acetate followed by Reynolds lead citrate for 4 and 3 min, respectively. The images were obtained with a Technai electron microscope (FEI Company: Hillsboro, OR). 


\subsubsection{Morphometric Analysis}

Electron micrograph images were taken from transverse sections of the tissue. Images of single cells (e.g. Fig. 2.3) were randomly taken from the sections. Images were taken such that different compartments of the cell were equally represented. For each muscle strip, five images were taken in which the nucleus was visible, five were taken in the region near the nuclear membrane which contains a high-density of mitochondria, and five were taken in regions between the nucleus and the end of the cell which contains mostly myofilaments. Three muscle strips per treatment group were examined for their myosin and actin filament densities, for a total of 45 cells per group. A blinded counter manually enumerated myosin thick filaments and measured the cross-sectional area of the cell by manual tracing using Image Pro Plus 5 (Media Cybernetics: Bethesda, MD). In protocol 1, the actin filaments were counted by randomly sampling small areas with the cells composed of only myofilaments. Within these small areas all of the actin filaments were counted and the area of sample determined by tracing. Thus, the actin filament density in Figure 2.4 is expressed as the filament density within the small sampled areas and not the whole cell. In protocol 2, actin filament density was determined from a computerized count of all filaments within a cross-sectional image of a cell. Images were initially processed such that areas of the cell without actin filaments were 'blacked out', such as along the cell membranes and dense bodies within the cells (Fig. 2.8). The altered images were then inverted such that the filaments became white dots, and using Image Pro Plus, an automated count of all of the white dots that were within the diameter range of 5-10 nm was accomplished. 


\subsubsection{Atomic Force Microscopy and Myosin Filament Formation in vitro}

Atomic force microscopy (AFM) was used to determine whether CD treatment had any effect on myosin filament formation in vitro (Fig. 2.7). The technique for purification and formation of myosin filaments has been described previously [7]. Briefly, myosin was obtained from bovine trachealis muscle and purified to a native-like myosin preparation. Purified myosin, in monomeric form, was dissolved in high ionic strength solution

composed of (mM): 5 EGTA, $1 \mathrm{Mg}^{2+}, 5 \mathrm{MgATP}, 10$ PIPES, and sufficient $\mathrm{KCl}$ to make $500 \mathrm{mM}$ ionic strength, with $\mathrm{pH}$ adjusted to 7.0 at $25^{\circ} \mathrm{C} .5 \mu \mathrm{M} \mathrm{CD}$ or $0.1 \%$ DMSO was added to this solution. The myosin in solution was dialyzed at $4{ }^{\circ} \mathrm{C}$ against a buffer containing $2 \mathrm{mM} \mathrm{MgCl} 2$ overnight; this caused the ionic strength to decrease from 500 $\mathrm{mM}$ to $88 \mathrm{mM}$.

The myosin filament containing solutions were deposited on freshly cleaved mica for AFM imaging. The mica surface was initially treated with $5 \mathrm{mM} \mathrm{MgCl}_{2}$ for 3 minutes, rinsed with distilled water, and dried with pressurized nitrogen gas. This process positively charges the mica, facilitating protein adhesion to the surface. Aliquots of 50 $\mu \mathrm{L}$ of sample were plated then after $5 \mathrm{~min}$, fixed with $10 \%$ gluteraldehyde for $3 \mathrm{~min}$. The mica was then rinsed with $90 \mathrm{mM}$ ammonium acetate and then dried again with nitrogen gas. The samples were placed under AFM (BioScope $^{\mathrm{TM}}$, Veeco Metrology Group: Chadds Ford, USA) for imaging in scanning mode. 


\subsubsection{Statistical Analysis}

Paired muscle strips were force-matched according to the force generated by EFS. Since pairs were removed from the same trachea, this force-matching provided a means of controlling for cross-sectional area of the muscle strips. Aggregate data were expressed as mean \pm SEM. Filament densities were calculated by dividing the total filament count by the cross-sectional area of the cell in $\mu \mathrm{m}^{2}$. Actin and myosin filament density data was compared in each cell compartment and combined to obtain a total cell density. Among cell compartments and for the combined filament densities for the cells, actin and myosin densities were compared by Student's t-tests using GraphPad Prism 5 (GraphPad Software, Inc.: La Jolla, CA). $\mathrm{p} \leq 0.05$ was considered to be sufficient to reject the null hypothesis.

\section{$2.3 \quad$ Results}

\subsubsection{Protocol 1: Treatment with $1 \mu M \mathrm{CD}$}

\subsubsection{Force Response to $1 \mu M$ Cytochalasin}

Treatment of the muscle with $1 \mu \mathrm{M}$ CD significantly reduced the force response to EFS, while the vehicle control group maintained force with no adverse response to the $0.1 \%$ DMSO (Fig. 2.2). Linear regression of force for the VC group followed the relationship of force $=0.001137 t+1$, while the exponential decay of the $\mathrm{CD}$ group followed the relationship of force $=0.6849 \mathrm{e}^{-0.0292 t}+0.3241$, where force was relative to $\mathrm{F}_{\max }$ and $t$ was time in minutes. At the final time point before fixation, $\mathrm{t}=65 \mathrm{~min}$, force for the $\mathrm{VC}$ group was $1.07 \mathrm{~F}_{\max }$ and $0.427 \mathrm{~F}_{\max }$ for the CD group. 


\subsubsection{Actin and Myosin Filament Density}

Cells that were fixed for EM exposed to either $\mathrm{CD}$ or the $\mathrm{VC}$ showed no obvious qualitative differences (not shown). Both actin and myosin filaments were readily identifiable from the EM images (Fig. 2.3). The actin filament density was sampled from myofilament-rich areas throughout the cell, as explained above. In the nuclear regions, mitochondrial regions, and myofilament regions, actin filament density was not significantly different when examined using this sampling technique (Fig. 2.4A). In the filamentous, mitochondrial and nuclear region, the VC group averaged $2462 \pm 183$, $2555 \pm 195$, and $2491 \pm 161$ actin filaments per $\mu \mathrm{m}^{2}$, respectively. While in the same regions the CD group averaged $2294 \pm 135,2166 \pm 140$, and $2342 \pm 222$ actin filaments per $\mu \mathrm{m}^{2}$, respectively. The composite actin filament density of all regions of the cells was $2274 \pm 92.2$ for the CD group vs. $2509 \pm 83.9$ per $\mu \mathrm{m}^{2}$ for the VC group (Fig. 2.4B).

Myosin filament density was determined by enumerating all myosin filaments from a cross-section (Fig. 2.5). Like the actin filament density, myosin filament density was not significantly different across all regions of the cell (Fig. 2.5A). In the filamentous, mitochondrial, and nuclear region, the VC group averaged $32.2 \pm 3.54,31.8 \pm 3.36$, and $18.7 \pm 2.72$ myosin filaments per $\mu \mathrm{m}^{2}$, respectively. While in the same regions the CD group averaged $36.6 \pm 3.31,36.5 \pm 3.32$, and $12.5 \pm 2.32$ myosin filaments per $\mu \mathrm{m}^{2}$, respectively. Likewise, the composite average of all cell regions was not different with the CD group averaging 28.5 \pm 3.45 and $\mathrm{VC}$ averaging 27.4 \pm 2.15 filaments per $\mu \mathrm{m}^{2}$ (Fig. $2.5 \mathrm{~B})$. 


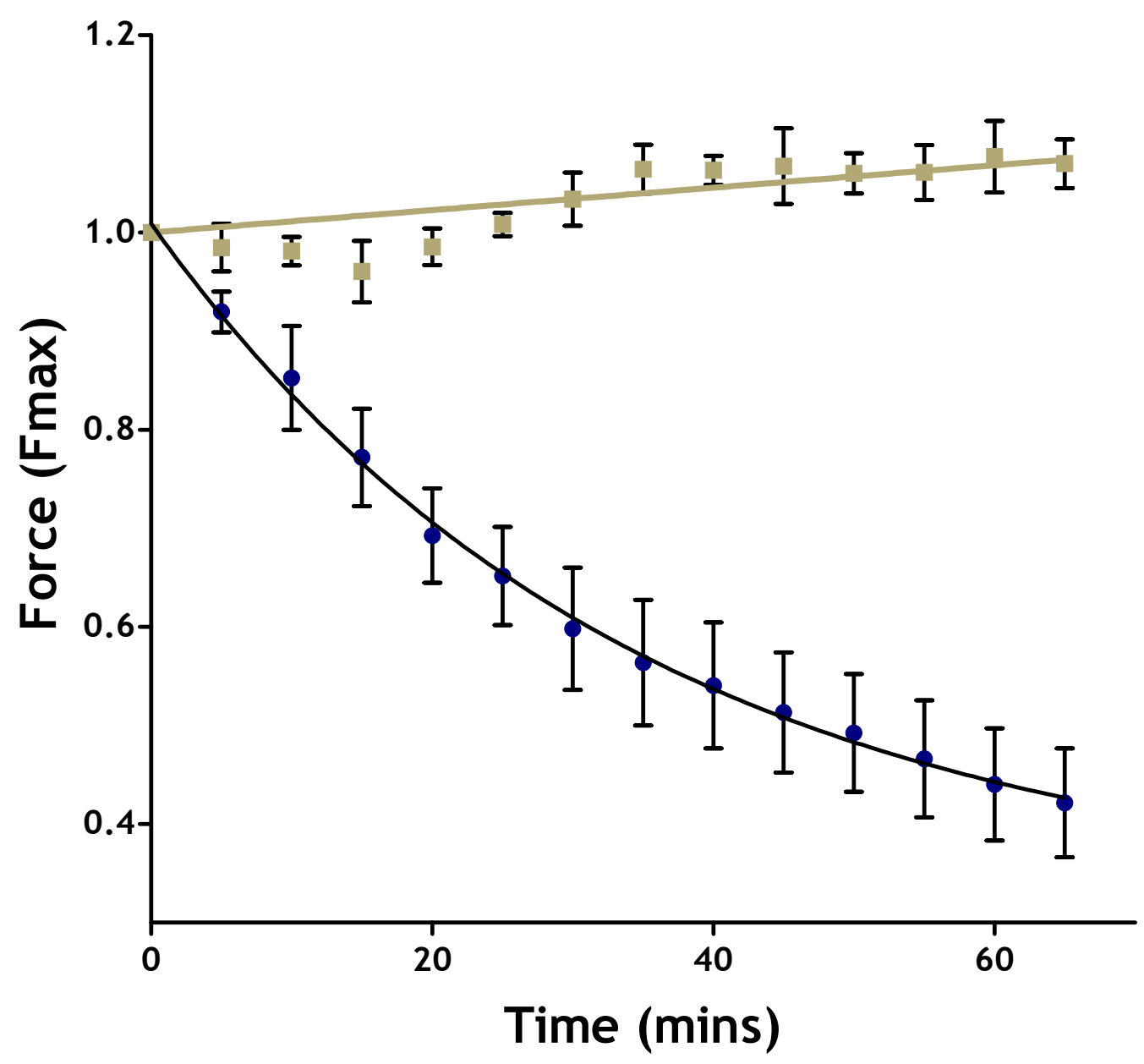

Figure 2.2 Force response to $1 \boldsymbol{\mu M}$ cytochalasin D. While the vehicle control group, indicated by squares, demonstrates an increase in force over a 65 min period, the cytochalasin D treated group, indicated by circles, demonstrates a significant decline in force. $\mathrm{n}=9$ for each group. Error bars indicate SEM. Both datasets are fit with regression lines, see text for details. 


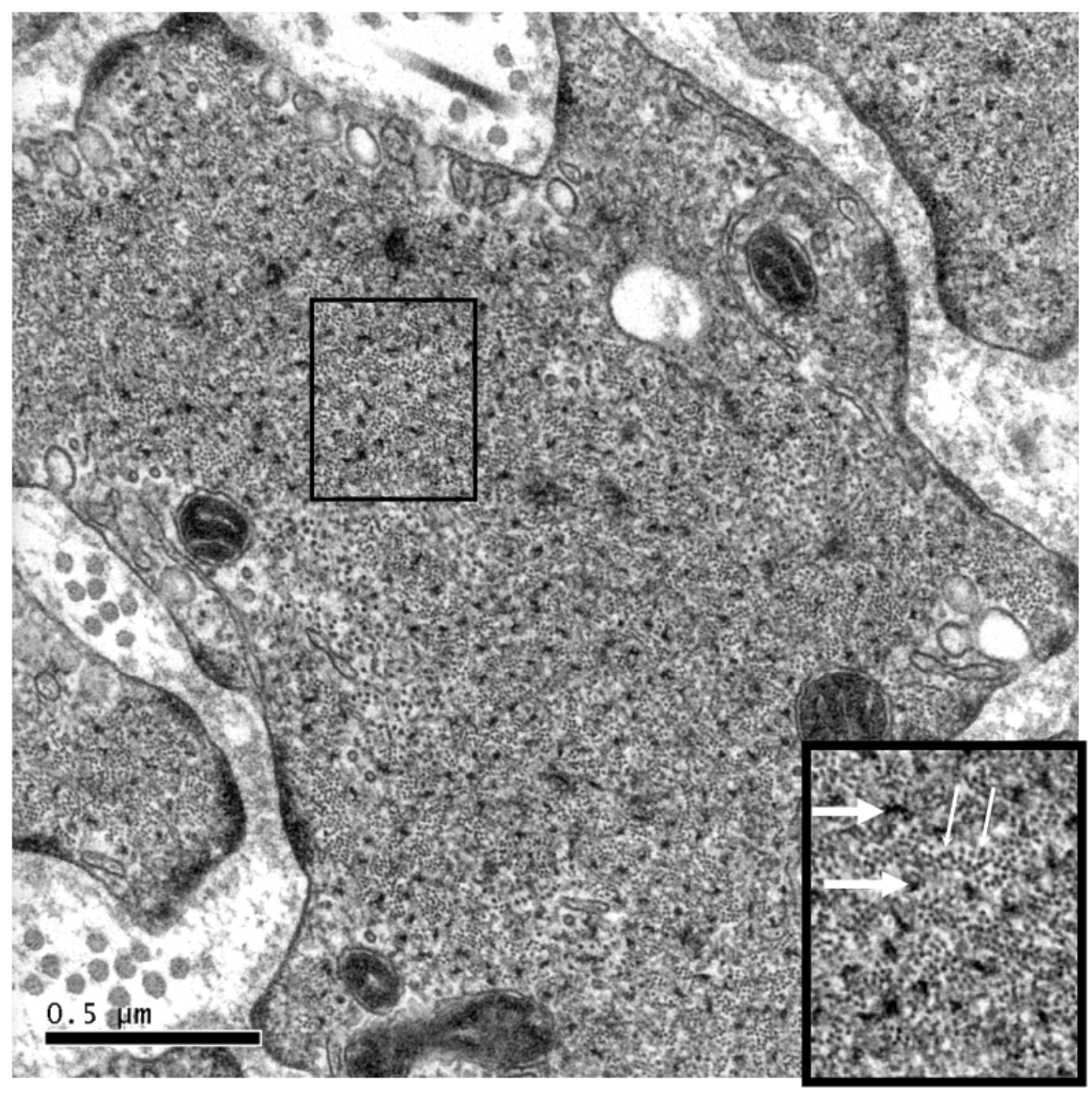

Figure 2.3 Electron micrograph of a cell treated with $1 \mu \mathrm{M}$ cytochalasin $\mathrm{D}$. The appearance of the CD treated cells was not obviously different than the VC cells. Inset: Myosin filaments (large arrows) and actin filaments (small arrows) were readily identifiable. Bar $=0.5 \mu \mathrm{m}$. 

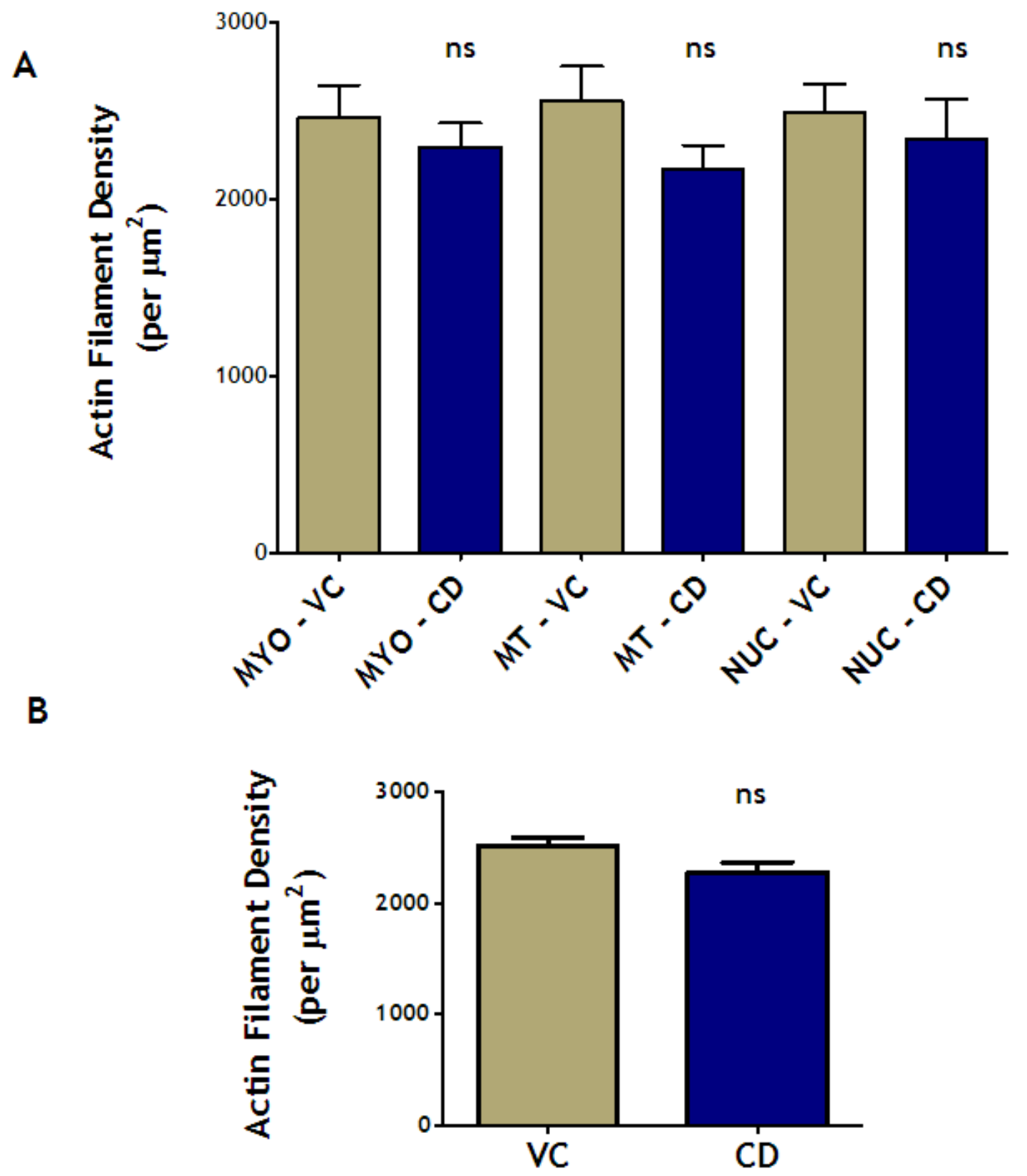

Figure 2.4 Actin filament density of smooth muscle treated with either the vehicle control (VC) or $1 \mu \mathrm{M}$ cytochalasin D (CD). Actin filament density was determined by sampling small filamentous regions within the cells. Within these small areas all of the actin filaments were counted and divided by the area of the sampled region. A) Cell sections were classified into three regions of the cell: the myofilamentous (MYO) region, 
the mitochondrial (MT) region, and the nuclear (NUC) region, see text for more detail. B) Filament density was combined from all regions into a composite average. $n=3$ per group. Error bars indicate SEM. ns = not significant; Student's t-test. 

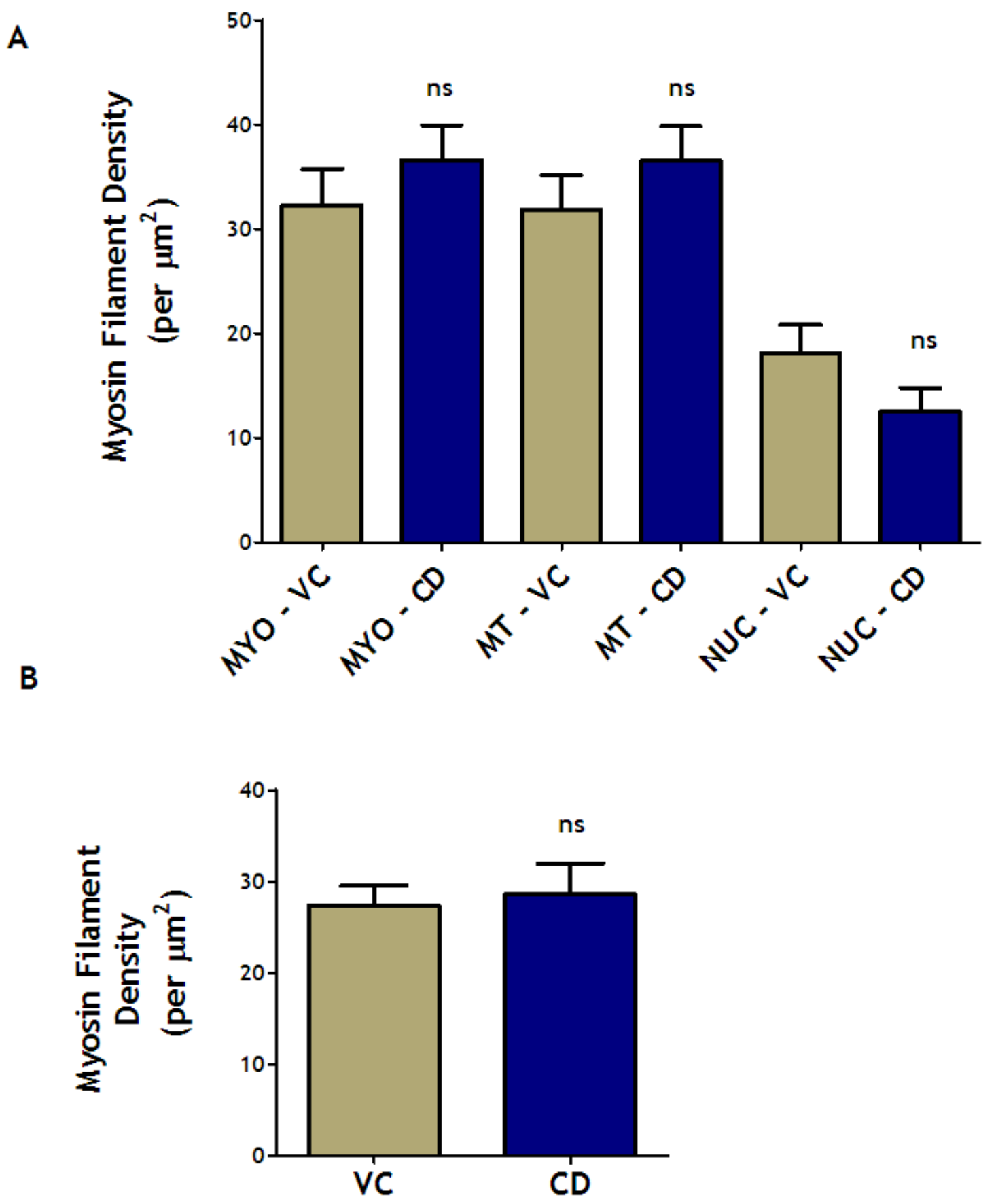

Figure 2.5 Myosin filament density of smooth muscle treated with either the vehicle control (VC) or $1 \boldsymbol{\mu M}$ cytochalasin D (CD). Myosin filament density was determined by manually counting all filaments within each cell and dividing by the cross-sectional area of the cell. A) Cell sections were classified into three regions of the cell: the 
myofilamentous (MYO) region, the mitochondrial (MT) region, and the nuclear (NUC) region, see text for more detail. B) Filament density was combined from all regions into a composite average. $\mathrm{n}=3$ per group. Error bars indicate SEM. ns = not significant; Student's t-test.

\subsubsection{Protocol 2: $5 \mu \mathrm{M}$ Cytochalasin and Length Oscillation}

Given that no observable differences were found from the ultrastructural analysis in response to $1 \mu \mathrm{M} \mathrm{CD}$, despite a significant decline in force, an alternative protocol was devised (Fig. 2.6). Since the hypothesis was to determine whether the actin filament lattice supports myosin filaments in a relaxed state, there was a possibility that despite destruction of the actin filaments, the myosin filaments would remain intact. Thus, this protocol sought to disturb the actin filaments by increasing the concentration of $\mathrm{CD}$ and mechanically perturbing myosin filaments that may remain in the absence of the lattice scaffold.

\subsubsection{Force Response to $5 \mu M$ Cytochalasin}

The force response following oscillation was similar to previous studies (Figure 1.2) [16] in which a exponential recovery was seen in the $\mathrm{VC}$ group. The $\mathrm{CD}$ group demonstrated no recovery and a significant reduction in force. This recovery period was described by a non-linear regression that followed a one-phase exponential association. For the VC group the relationship was described as force $=0.967-0.177 \mathrm{e}^{-0.275 t}$ and for the CD group as force $=0.0070+0.3406 \mathrm{e}^{-0.02540 t}$, where force was relative to $\mathrm{F}_{\max }$ and $t$ was time in minutes. At the end of the 20 min recovery period and just prior to EM fixation, the VC 
group averaged $0.966 \mathrm{~F}_{\max }$ and the $\mathrm{CD}$ group averaged $0.212 \mathrm{~F}_{\max }$. It was important, as a control, to demonstrate that myosin filaments were not affected directly by CD treatment (Fig. 2.7). The AFM images demonstrated no qualitative difference between myosin filaments grown in vitro in the presence of the $\mathrm{VC}$ or $5 \mu \mathrm{M} \mathrm{CD}$.

\subsubsection{Actin and Myosin Filament Density}

In protocol 1, actin filament density was determined by random sampling of small regions within cells. Since the results of this sampling did not demonstrate any differences, protocol 2 used computerized counting to enumerate all filaments within the cell (Fig. 2.8). The results of this counting are shown in Figure 2.8. At the ends of the cells where myofilaments predominate the cell area, actin filament density was found to be significantly greater in the $\mathrm{CD}$ treated group than in the VC group (Fig. 2.9A) (Student's t-test: $\mathrm{p}<0.05$ ). In the filamentous region the VC group averaged $824 \pm 72$ per $\mu \mathrm{m}^{2}$ while the CD group averaged $1271 \pm 48$ actin filaments per $\mu \mathrm{m}^{2}$. The other two regions of the cell did not demonstrate any differences. In the mitochondrial region and nuclear regions, the VC group averaged $1035 \pm 34$ and $1060 \pm 53$ actin filaments per $\mu \mathrm{m}^{2}$, respectively. While in the same regions the CD group averaged $943 \pm 58$ and $1071 \pm 79$ actin filaments per $\mu \mathrm{m}^{2}$, respectively. When all cell regions were combined the composite average demonstrated that $\mathrm{CD}$ treated cells had a greater actin filament density than the VC, with CD averaging $1095 \pm 44$ vs. VC averaging $973 \pm 37$ filaments per $\mu \mathrm{m}^{2}$ (Fig. 2.9B) (Student's t-test: $\mathrm{p}<0.05$ ).

Myosin filament density was determined using the same method as protocol 1 . Filament densities were determined in individual regions of the cells (Fig. 2.10A), with only the 
nuclear region demonstrating a significant difference (Student's t-test: $\mathrm{p}<0.01$ ). The VC group averaged $26.0 \pm 2.07$ vs. the $C D$ group which averaged $13.6 \pm 1.47$ filaments per $\mu \mathrm{m}^{2}$, in the nuclear region. In the filamentous and mitochondrial region, the VC group averaged 27.6 \pm 2.19 and $22.6 \pm 2.60$ myosin filaments per $\mu \mathrm{m}^{2}$, respectively. In the same regions, the CD group averaged $27.1 \pm 3.58$ and $23.2 \pm 3.24$ myosin filaments per $\mu \mathrm{m}^{2}$, respectively. The composite average was not significantly different with the VC group averaging 25.4 \pm 1.07 and the $C D$ group averaging 21.3 \pm 1.81 filaments per $\mu \mathrm{m}^{2}$ (Fig. 2.10B). 


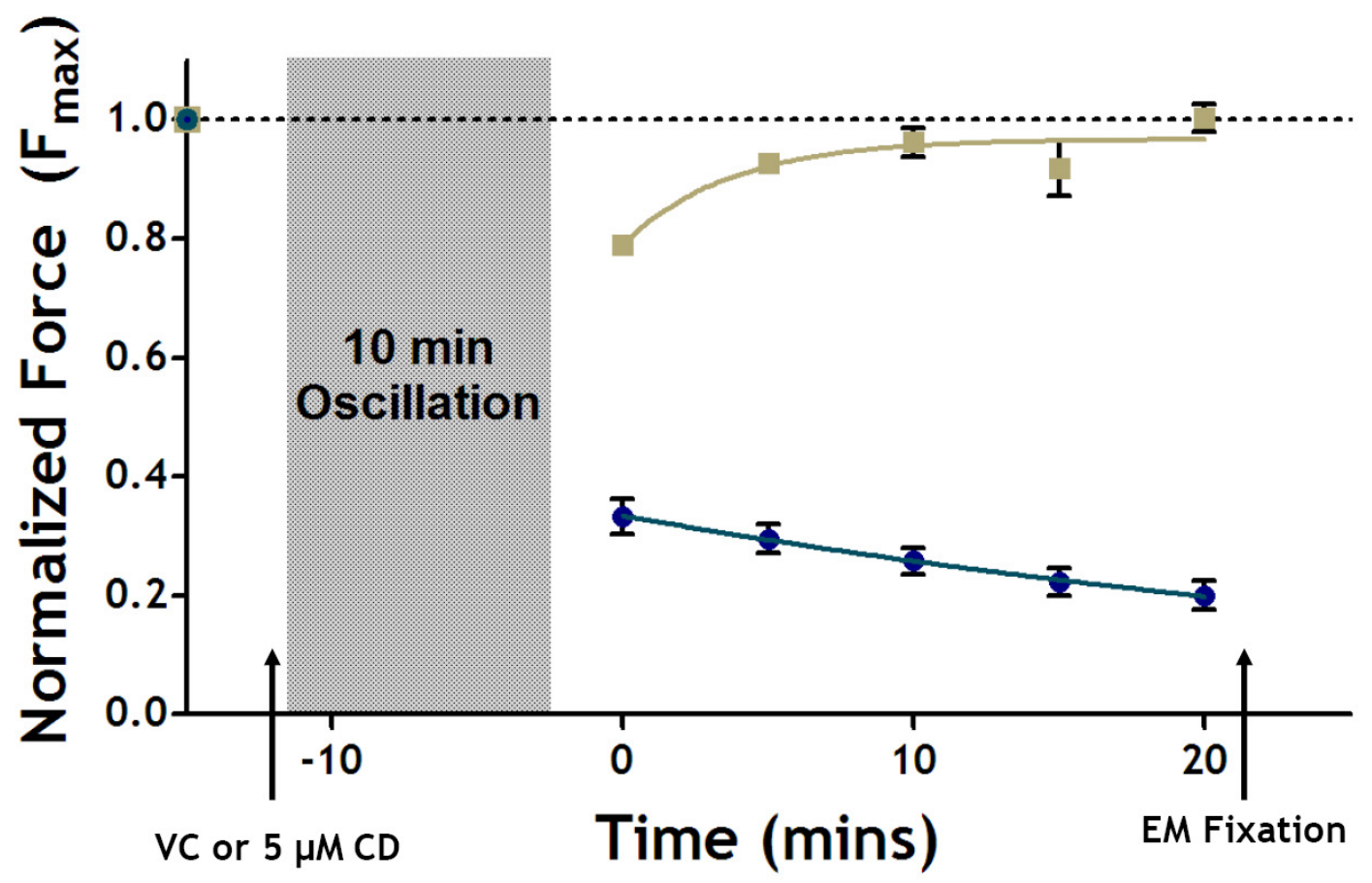

Figure 2.6 Treatment with $5 \mu \mathrm{M}$ cytochalasin D during length oscillation and recovery. Muscle strips were treated with either the vehicle control (VC - squares) or cytochalasin D (CD - circles) immediately prior to length oscillation. The length oscillation consisted of a 10 minute, $0.25 \mathrm{~Hz}$ sinusoidal length oscillation with a amplitude of $30 \% \mathrm{~L}_{\text {ref. }}$. After the oscillation, the muscle strips were allowed to recover for five contractions before fixation for EM. The muscle was still treated with $\mathrm{CD}$ or the VC during recovery. Both datasets were fit with exponential regression curves, see text for details. $\mathrm{n}=5$ for each group. Error bars indicate SEM. 


\section{A: VC}

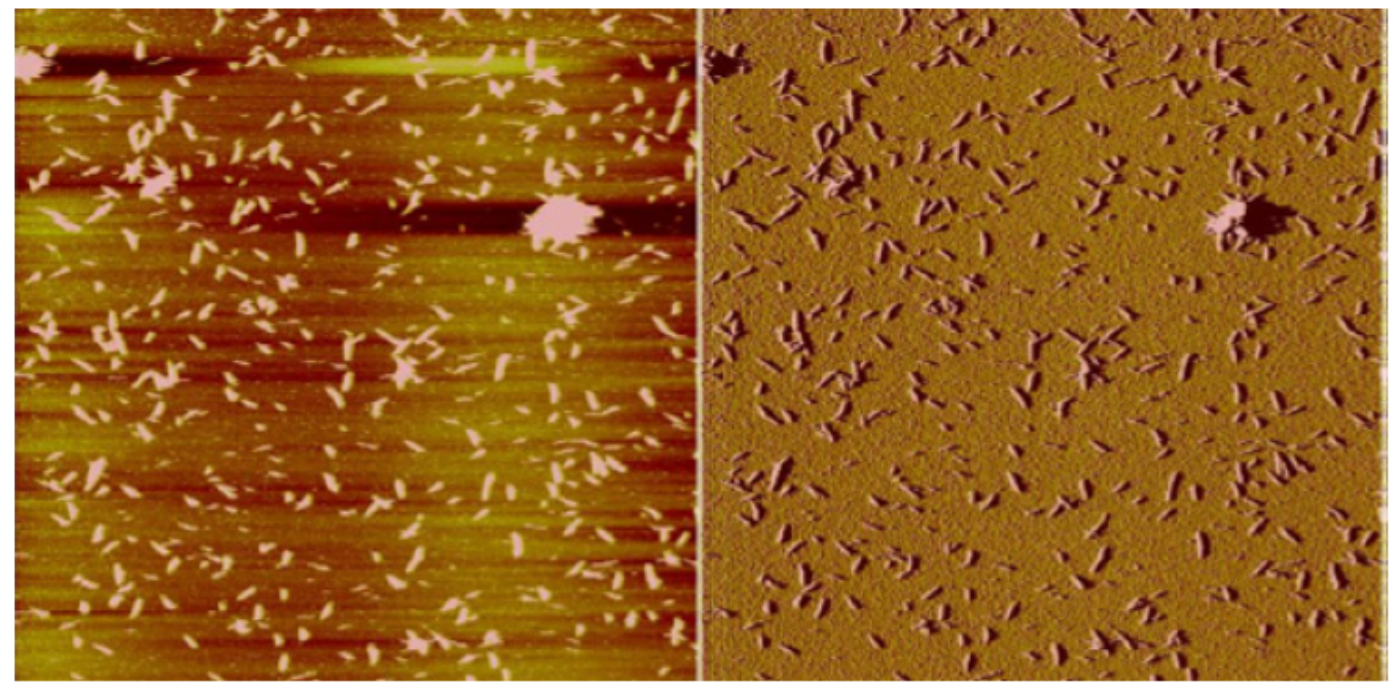

\section{B: $C D$}
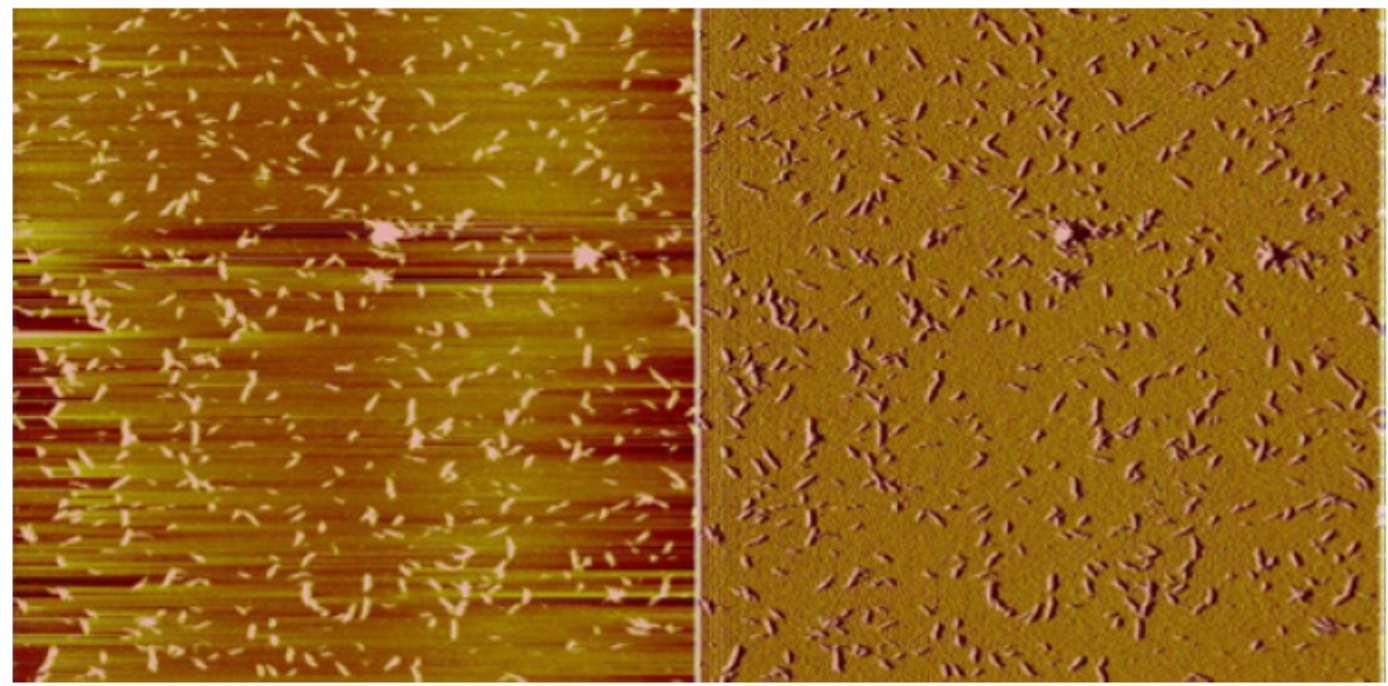

Figure 2.7 Myosin filaments polymerized in vitro imaged by atomic force

microscopy. The filaments were grown either in the presence of the VC or $5 \mu \mathrm{M} \mathrm{CD}$. The images on the left are the height images, in which objects between $0-20 \mathrm{~nm}$ are represented by a brightness scale. Objects nearer the surface are dark (red color) while objects further from the mica surface are brighter (yellow or white color). The images on the right are the same as the left but produced from the displacement signal of the 
scanning tip of the AFM. This type of image eliminates the effect of the unevenness of the mica surface seen on the left, producing a clearer image. Image size: 6.7 x $6.7 \mu \mathrm{m}$. 
Figure 2.8 EM image processing for computerized actin filament counting. EM images of cells (a) were processed by manually 'blacking out' regions of the cell that did not contain actin filaments (b). After image inversion (c) all objects within the cell between 5-10 nm were counted. See text for further details. 

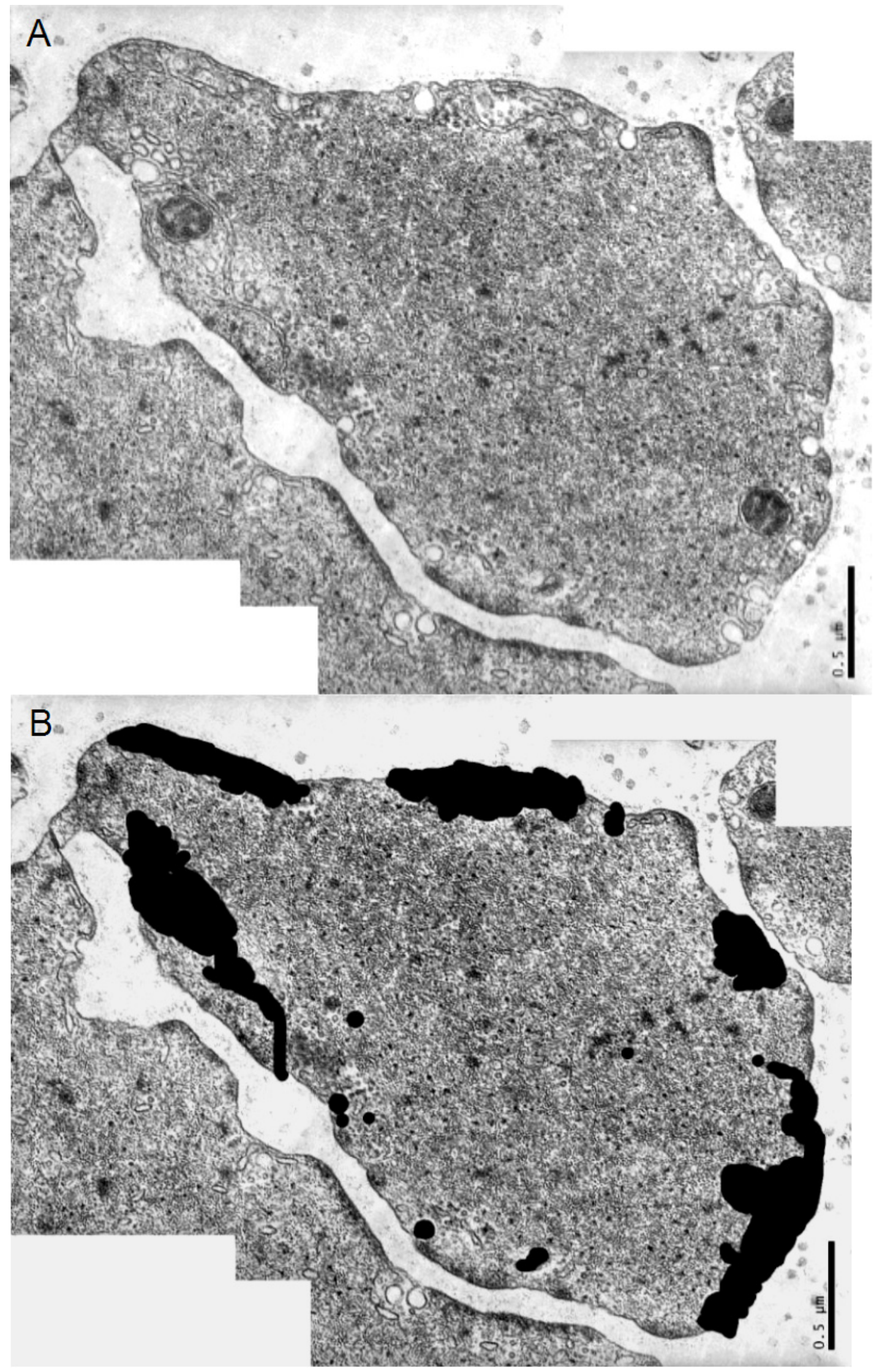


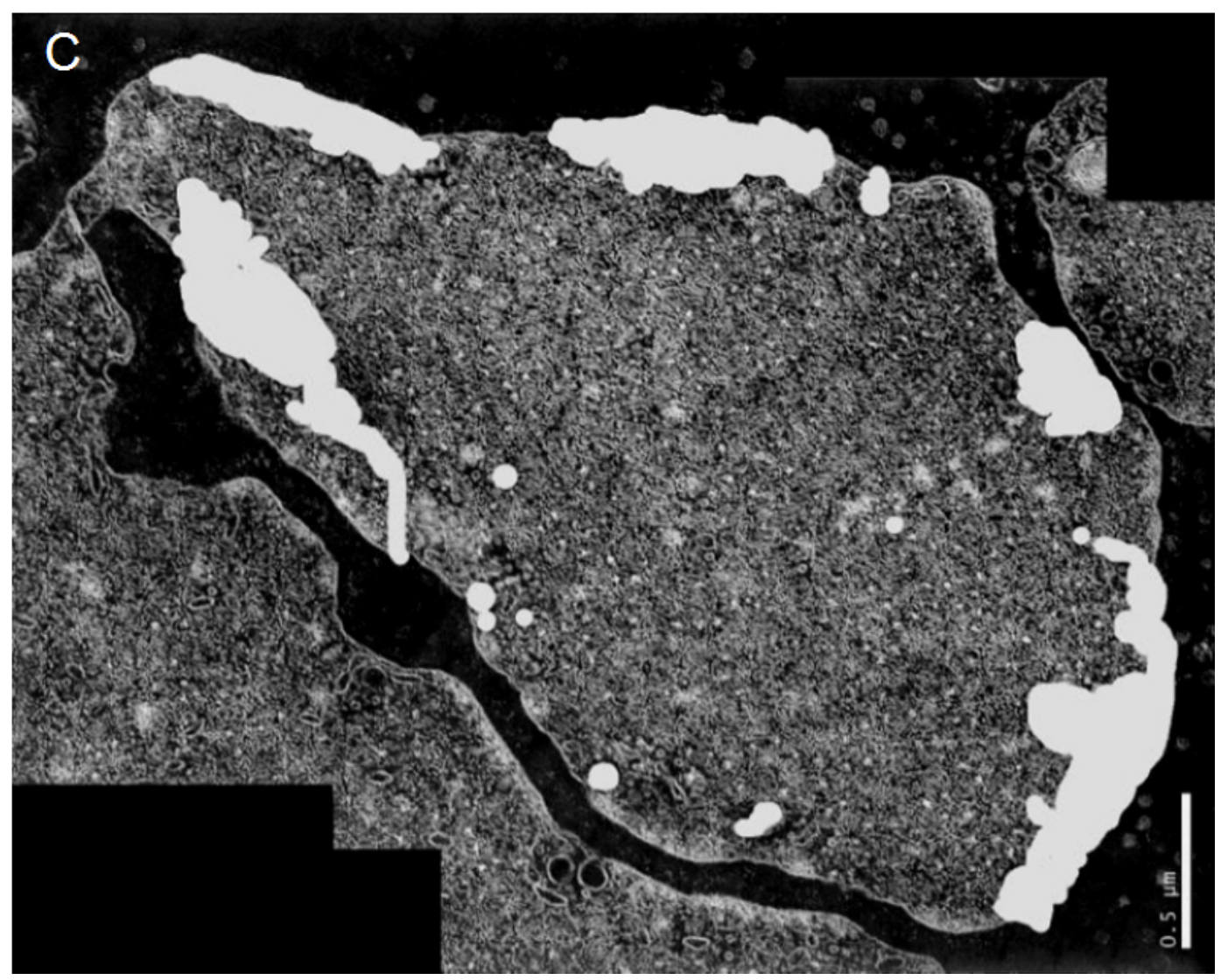


A

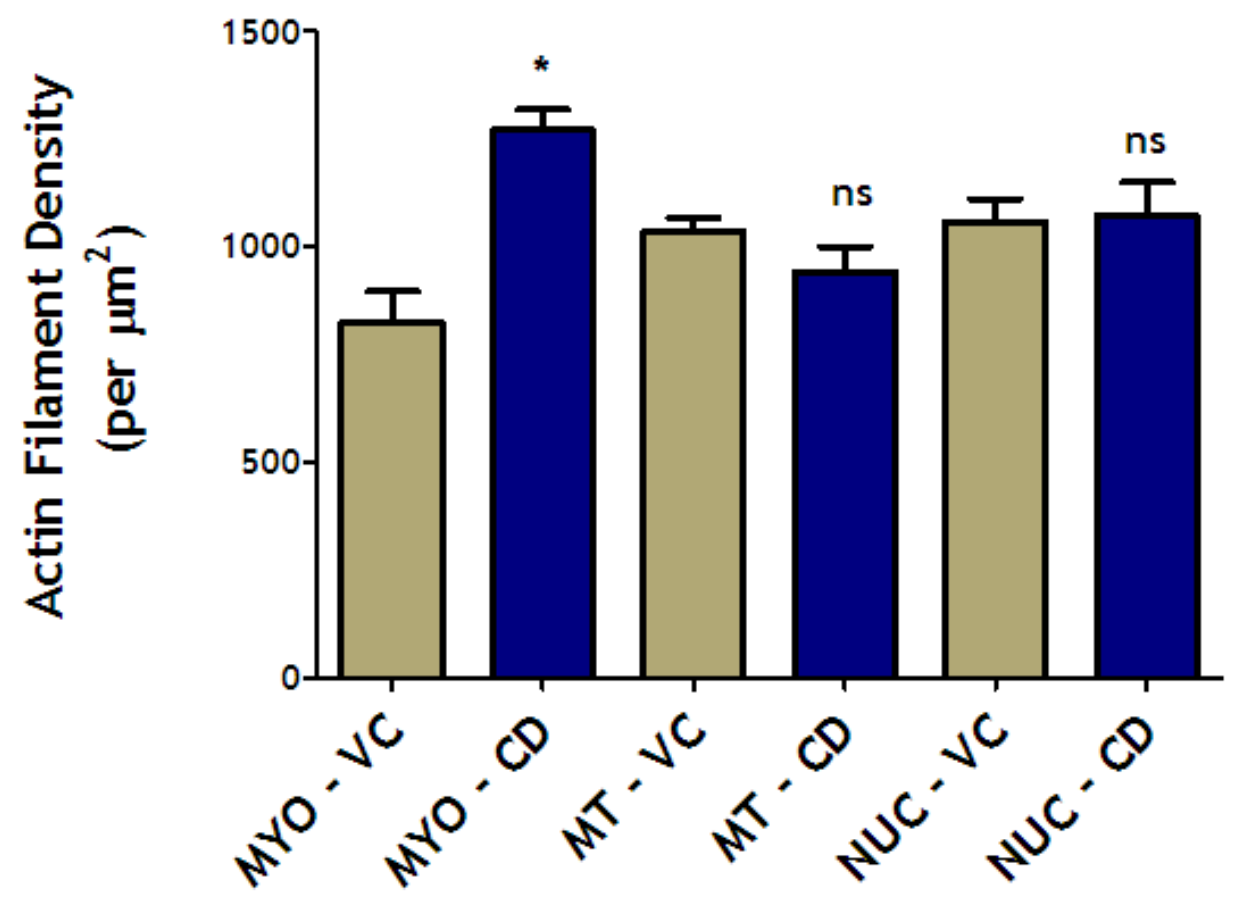

B

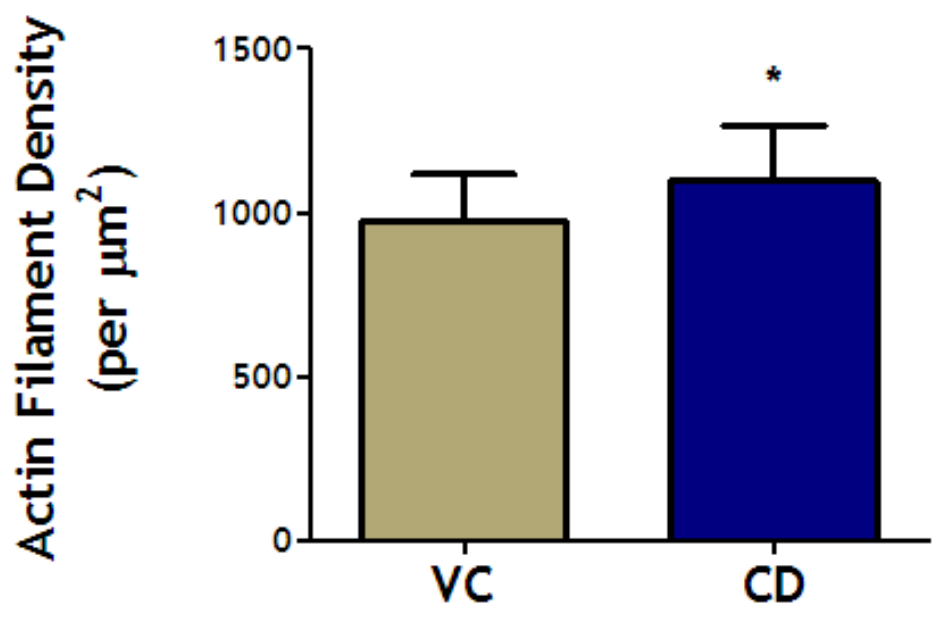

Figure 2.9 Actin filament density of smooth muscle treated with either the vehicle control (VC) or $5 \boldsymbol{\mu M}$ cytochalasin D (CD). Actin filament density was determined by computerized counting of filaments (see Fig. 2.8). The total number of filaments was divided by the cross-sectional area of the cell. A) Cell sections were classified into three 
regions of the cell: the myofilamentous (MYO) region, the mitochondrial (MT) region, and the nuclear (NUC) region, see text for more detail. B) Filament density was combined from all regions into a composite average. $n=3$ per group. Error bars indicate SEM. ns = not significant; *: $p<0.05$; Student's t-test. 
A

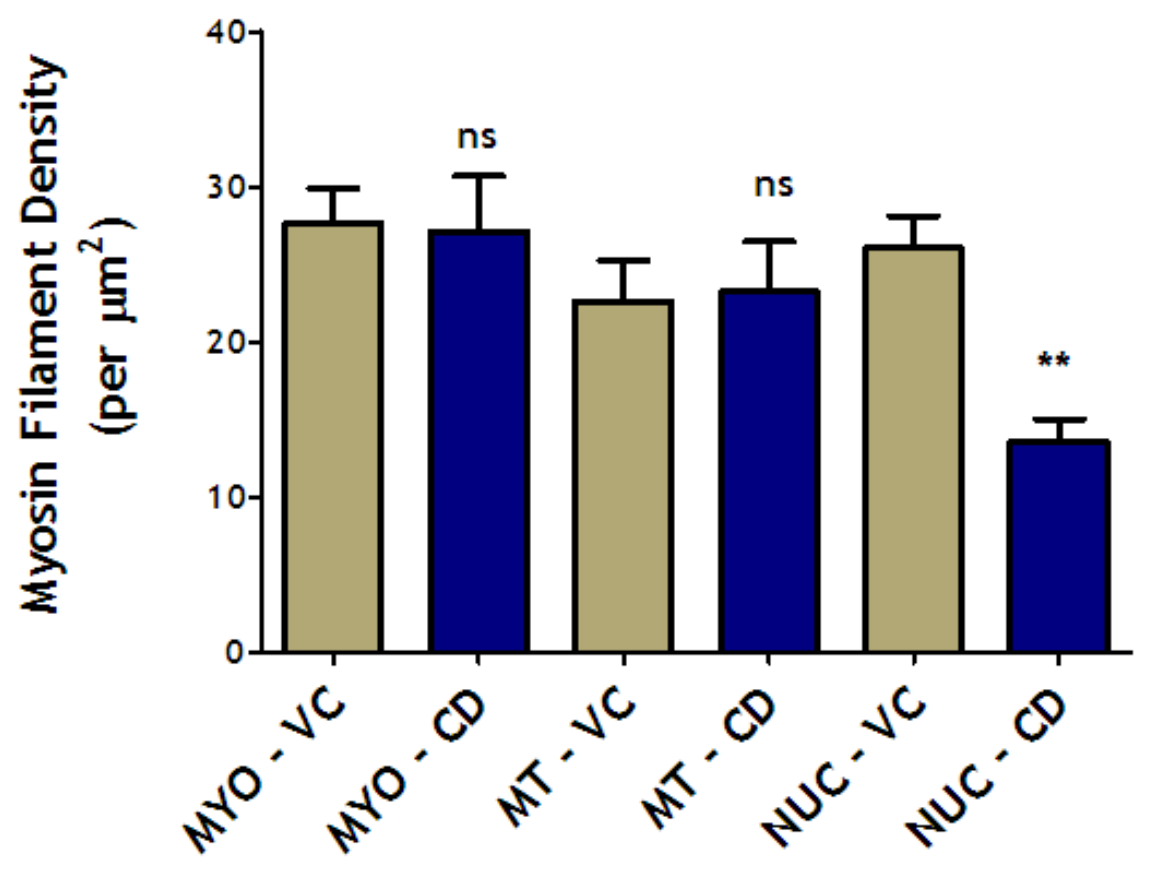

B

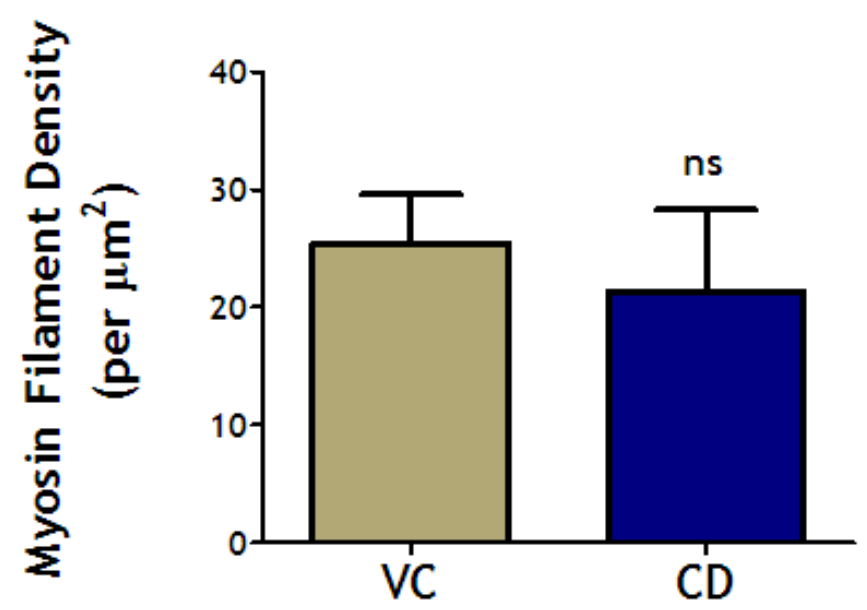

Figure 2.10 Myosin filament density of smooth muscle treated with either the vehicle control (VC) or $5 \mu$ M cytochalasin D (CD). Myosin filament density was determined by manually counting all filaments within each cell and dividing by the crosssectional area of the cell. A) Cell sections were classified into three regions of the cell: the myofilamentous (MYO) region, the mitochondrial (MT) region, and the nuclear 
(NUC) region, see text for more detail. B) Filament density was combined from all regions into a composite average. $\mathrm{n}=3$ per group. Error bars indicate SEM. $\mathrm{ns}=\mathrm{not}$ significant; $^{* *}: \mathrm{p}<0.01$; Student's t-test.

\subsection{Discussion}

Cytochalasin D is a well-studied antagonist of actin filament polymerization $[13,17,18]$ that has been shown to cause marked depolymerization in various non-contractile cells $[17,19]$ and cultured smooth muscle cells [20]. We postulated that it would be a useful agent to test the hypothesis that the actin filament lattice is necessary for the presence of myosin filaments in relaxed smooth muscle cells, because it is cell permeable and has an observable, dramatic effect on force (Fig. 2.2). CD is thought to be the most potent actin binding cytochalasin. Even at the lower concentration of $1 \mu \mathrm{M}, \mathrm{CD}$ reduced force markedly by $61 \%$ compared to the $\mathrm{VC}$, a change that suggests major changes to the contractile filaments within the cells. Therefore, it was surprising to discover virtually no differences in actin and myosin filament densities (Figs. 2.4 and 2.5).

It is possible that the methodology employed was inadequate to observe changes in actin filaments. Since cross-sectional images of cells were used it is not entirely clear how changes would be manifested in actin filament density. While the depolymerizing action of $\mathrm{CD}$ would lead to a population of predominately shorter actin filaments, it is unknown whether there would be more or less filaments. This process would depend on the shifting of the equilibrium between F-actin and G-actin caused by CD. In theory, CD could cap 
actin filaments leading to filament truncation without changing the number of filaments, however, the presence of shorter filaments without changes in filament number would likely lead to a decrease in filament density simply because of the decreased likelihood of imaging a shorter filament versus a longer one. Since the fraction of G-actin was not determined, it is impossible to predict whether $\mathrm{CD}$ would shift the equilibrium towards increased filament density but decreased filament length or the reverse, fewer and longer filaments.

Without changes to actin and myosin filament density despite the significant decline in force, it was uncertain whether the actin filament count was accurate given the small sampling areas examined. Since the actin filament density counted by sampling in the first protocol (Fig. 2.4) was much greater than the usual $\sim 30: 1$ ratio between actin and myosin filaments, the sampling areas overestimated the number of actin filaments in the entire cell cross-section. Thus, to remove any possible power issues related to sampling, a second protocol was devised and a full count of actin filaments within the cross-sectional area of the cells was determined using a computerized enumeration technique (Fig. 2.8). Also, the $\mathrm{CD}$ concentration was increased to $5 \mu \mathrm{M}$ to exaggerate the effect of $\mathrm{CD}$ on force and, hopefully, actin filament depolymerization. Since no differences were observed in myosin filament density in protocol one (Fig. 2.5), a 10 minute length oscillation was added to disrupt any myosin filaments that may remain despite the destruction of the actin filament lattice. This technique has been shown to cause a reduction in force that coincides with a reduction in myosin filament density (Fig. 1.2) [16]. 
Using the computerized counting technique, actin filament density was found to be increased in the myofilamentous regions of the cells but not the mitochondrial or nuclear regions (Fig. 2.9A). This difference led to an overall difference in the composite density average (Fig. 2.9B). This observation could come about if $\mathrm{CD}$ led to an increase number of actin filaments of shorter length. While the increase in density could be explained by an increase in actin filament length alone, this is likely not the case, given the purported mechanism of $\mathrm{CD}$ on actin filaments. Myosin filament density was not different in the myofilamentous and mitochondrial regions but significantly lower in the nuclear regions of the cells (Fig. 2.10A). Overall, the composite myosin filament density through all regions was not significantly different. Since there was no association of changes in actin filament density in the nuclear regions with myosin filament density, it is unclear why such a difference in myosin filaments would be observed. It is possible that the results are due to sampling issues given that the cross-sections of the nucleus often have limited area for filaments, given that the nucleus covers $\sim 80 \%$ of the cell area in these cross-sections.

These results suggest that the actin filament lattice may not stabilize myosin filaments in relaxed cells, an argument that may be supported by the increase in actin filaments in the myofilamentous region following $5 \mu \mathrm{M} \mathrm{CD}$ treatment (Fig. 2.9A), but no resulting changes in myosin filament density in the same region (Fig. 2.10A). Despite the previously mentioned limitations to filament density counting, it would be prudent to examine whether $\mathrm{CD}$ has an alternative mechanism of action. $\mathrm{CD}$ toxicity may also explain the decrease in force that was observed, however, changes related to cell damage 
were not observed under EM. While the use of CD to disrupt cytoskeletal networks in other cell types such as fibroblasts and epithelial cells is well documented [17, 19], studies involving smooth muscle cells have mostly been on cultured cells. As with fibroblasts, smooth muscle cells in culture undergo stress fiber disruption in response to relatively low concentrations of $\mathrm{CD}$, around $1 \mu \mathrm{M}[20,22]$. These observations are dramatic and can be visualized using confocal microscopy.

The results of studies involving intact muscle bundles have been far more ambiguous. Mehta and Gunst [3] examined intact ASM bundles by treating with a relatively high concentration of $10 \mu \mathrm{M} C D$. Similar to this study, they found a significant reduction in force generation with $\mathrm{CD}$ treatment, but no changes in actin filaments when examined under electron microscopy (longitudinal sections) or under confocal microscopy with rhodamine-phalloidin actin filament staining [3]. In addition, they employed the DNase I activity assay to determine the G-actin content of the muscle strips. Starting at a normalized level of $100 \%$ G-actin content in the unstimulated muscle, addition of acetylcholine (ACh) caused a reduction to $60 \%$ G-actin content. This confirmed the well documented process of actin polymerization during contraction. Treatment with latrunculin A, a noted sequesterer of G-actin, caused no increase in actin filaments following $\mathrm{ACh}$ contraction. When $10 \mu \mathrm{M} \mathrm{CD}$ was added along with $\mathrm{ACh}$, actin polymerization occurred, with a similar reduction to $60 \%$ G-actin content. Thus, the presence of $\mathrm{CD}$ did not reduce contraction mediated actin filamentogenesis. From these data they concluded that "the effects of these agents [latrunculin A and cytochalasin D] 
on force are unlikely to result from the disruption of actin filament integrity or disorganization of the contractile apparatus" [3].

One other study examined the ultrastructure of arterial smooth muscle cells in response to a high dose of $\mathrm{CD}, 10 \mu \mathrm{g} / \mathrm{mL}$ which equates to $19.7 \mu \mathrm{M}$ [23]. By fixing the cells for $\mathrm{EM}$ during contraction when $\mathrm{CD}$ had caused a total abolition of force, they observed a near $40 \%$ decrease in actin filament density. While significant, the ultrastructural changes observed cannot entirely explain the dramatic decrease in force. Taken together with the data from this study, it would suggest that CD has an effect on force independent of an effect on actin filaments. Indeed, an in vitro study of vascular smooth muscle actomyosin interaction supports such a hypothesis [24]. They found that "cytochalasin D (10 $\mu \mathrm{M})$ did not completely depolymerize actin filament[s], while it completely inhibited the smooth muscle contraction." From these results they suggest that CD likely acts directly at the site of actomyosin interaction. While they found no changes in rMLC phosphorylation due to $C D$, they did find that $C D$ slowed the attachment of myosin to actin based on a measure of the turbidity of native actomyosin.

Another possible mechanism leading to force attenuation would be an affect of CD on calcium signaling. $100 \mu \mathrm{M}$ CD treatment of cultured vascular smooth muscle cells has been shown to interfere with L-type $\mathrm{Ca}^{2+}$ channel current, presumably through a spatial dissociation of the channel from the cytoskeleton [25]. Again, it is unclear whether a similar mechanism would be present in intact muscle strips since we did not see reductions in actin filaments with $\mathrm{CD}$ treatment. However, it is possible that $\mathrm{CD}$ may 
have a direct effect on calcium entry into the cell independent of its affects on actin filaments. Further investigation is needed in this area.

In conclusion, in this study we sought to determine whether the actin filament lattice supports the integrity of myosin filaments in relaxed smooth muscle cells. However, CD was not the proper agent to address this question, given that it had a significant effect on force but no observable effect on actin filaments. To appropriately answer this question these experiments need to be repeated with an effective actin depolymerizing agent (see Chapter Six for further discussion). 


\subsection{References}

1. Pratusevich VR, Seow CY, Ford LE. Plasticity in canine airway smooth muscle. J.Gen.Physiol. 1995; 105: 1: 73-94.

2. Seow CY. Myosin filament assembly in an ever-changing myofilament lattice of smooth muscle. Am.J.Physiol.Cell.Physiol. 2005; 289: 6: C1363-8.

3. Mehta D and Gunst SJ. Actin polymerization stimulated by contractile activation regulates force development in canine tracheal smooth muscle. J.Physiol. 1999; 519 Pt 3: 829-840.

4. Zhang W and Gunst SJ. Dynamic association between alpha-actinin and beta-integrin regulates contraction of canine tracheal smooth muscle. J.Physiol. 2006; 572: Pt 3: 659-676.

5. Herrera AM, Martinez EC, Seow CY. Electron microscopic study of actin polymerization in airway smooth muscle. Am.J.Physiol.Lung Cell.Mol.Physiol. 2004; 286: 6: L1161-8.

6. Suzuki H, Onishi H, Takahashi K, Watanabe S. Structure and function of chicken gizzard myosin. J.Biochem. 1978; 84: 6: 1529-1542.

7. Ip K, Sobieszek A, Solomon D, Jiao Y, Pare PD, Seow CY. Physical integrity of smooth muscle myosin filaments is enhanced by phosphorylation of the regulatory myosin light chain. Cell.Physiol.Biochem. 2007; 20: 5: 649-658.

8. Trybus KM and Lowey S. Assembly of smooth muscle myosin minifilaments: effects of phosphorylation and nucleotide binding. J.Cell Biol. 1987; 105: 6 Pt 2: 3007-3019.

9. Craig R and Megerman J. Assembly of smooth muscle myosin into side-polar filaments. J.Cell Biol. 1977; 75: 3: 990-996.

10. Sobieszek A and Small JV. Regulation of the actin-myosin interaction in vertebrate smooth muscle: activation via a myosin light-chain kinase and the effect of tropomyosin. J.Mol.Biol. 1977; 112: 4: 559-576.

11. Mahajan RK, Vaughan KT, Johns JA, Pardee JD. Actin filaments mediate Dictyostelium myosin assembly in vitro. Proc.Natl.Acad.Sci.U.S.A. 1989; 86: 16: 6161-6165.

12. Mahajan RK and Pardee JD. Assembly mechanism of Dictyostelium myosin II: regulation by $\mathrm{K}+, \mathrm{Mg} 2+$, and actin filaments. Biochemistry 1996; 35: 48: 1550415514. 
13. Cooper JA. Effects of cytochalasin and phalloidin on actin. J.Cell Biol. 1987; 105: 4: 1473-1478.

14. Schliwa M. Action of cytochalasin D on cytoskeletal networks. J.Cell Biol. 1982; 92: 1: 79-91.

15. Bosse Y, Chin LY, Pare PD, Seow CY. Chronic Activation in Shortened Airway Smooth Muscle: A Synergistic Combination Underlying Airway Hyperresponsiveness? Am.J.Respir.Cell Mol.Biol. 2009; .

16. Kuo KH, Wang L, Pare PD, Ford LE, Seow CY. Myosin thick filament lability induced by mechanical strain in airway smooth muscle. J.Appl.Physiol. 2001; 90: 5: 1811-1816.

17. Schliwa M. Action of cytochalasin D on cytoskeletal networks. J.Cell Biol. 1982; 92: 1: 79-91.

18. Goddette DW and Frieden C. Actin polymerization. The mechanism of action of cytochalasin D. J.Biol.Chem. 1986; 261: 34: 15974-15980.

19. Wakatsuki T, Schwab B, Thompson NC, Elson EL. Effects of cytochalasin D and latrunculin B on mechanical properties of cells. J.Cell.Sci. 2001; 114: Pt 5: 10251036.

20. An SS, Laudadio RE, Lai J, Rogers RA, Fredberg JJ. Stiffness changes in cultured airway smooth muscle cells. Am.J.Physiol.Cell.Physiol. 2002; 283: 3: C792-801.

21. Puszkin E, Puszkin S, Lo LW, Tanenbaum SW. Binding of cytochalasin D to platelet and muscle myosin. J.Biol.Chem. 1973; 248: 22: 7754-7761.

22. Nagayama $\mathrm{K}$ and Matsumoto T. Estimation of single stress fiber stiffness in cultured aortic smooth muscle cells under relaxed and contracted states: Its relation to dynamic rearrangement of stress fibers. J.Biomech. 2010;

23. Adler KB, Krill J, Alberghini TV, Evans JN. Effect of cytochalasin D on smooth muscle contraction. Cell.Motil. 1983; 3:545-51.

24. Saito SY, Hori M, Ozaki H, Karaki H. Cytochalasin D inhibits smooth muscle contraction by directly inhibiting contractile apparatus. J.Smooth Muscle Res. 1996; 32: 2: 51-60.

25. Nakamura M, Sunagawa M, Kosugi T, Sperelakis N. Actin filament disruption inhibits L-type $\mathrm{Ca}^{2+}$ channel current in cultured vascular smooth muscle cells. Am J Physiol Cell Physiol. 2000; 279: C480-C487. 


\section{CHAPTER 3. THE NUMBER OF MYOSIN FILAMENTS IN POTASSIUM CHLORIDE STIMULATED SMOOTH MUSCLE DOES NOT CORRELATE TO THE FORCE RESPONSE $^{1}$}

\subsection{Introduction}

Smooth muscle cells are present in many organ systems and provide vital physiological functions. These wide-ranging functions include focusing the lens of the eye, changing the volume of the urinary bladder, regulating blood pressure, and peristalsis of the digestive system. Smooth muscle is also present throughout the respiratory system where its function is the subject of great debate [1,2]. Regardless, airway smooth muscle (ASM) plays a critical role in airway narrowing in asthma. Given the pathophysiological importance of smooth muscle contraction, surprisingly little is understood about the molecular mechanisms by which smooth muscle cells generate force.

It is generally accepted that the sliding filament $[3,4]$ and crossbridge theories [5] developed for skeletal muscle are also fundamental mechanisms of contraction in smooth muscle [6], however it is still unclear how the cell is organized to produce force. The generally accepted model of the smooth muscle 'sarcomere' is thought to be composed of at least two actin filaments flanking a side-polar myosin filament [7-9]. Since the filaments are not arrayed in an organized fashion like those in skeletal muscle, determining how and where contractile units are arranged remains elusive. Furthermore,

\footnotetext{
${ }^{1}$ A version of this chapter will be submitted for publication. Chin LYM, Bossé Y, Kuo K, Jiao Y, Solway J, Paré PD, Seow CY. (2010) The Number of Myosin Filaments in Potassium Chloride Stimulated Smooth Muscle Does Not Correlate to the Force Response.
} 
contractile filaments are capable of assembly and disassembly (referred to as plasticity) during the contraction-relaxation cycle $[10,11]$. This dynamic assembly of actin and myosin filaments in response to a contractile stimulus has been observed by electron microscopy (EM) [10]. The process by which myosin filaments assemble and disassemble has been termed myosin evanescence [12-15]. While the mechanism is still unclear, myosin filaments have been shown to have increased stability and the monomers which make up the filaments are more tightly packed when the regulatory myosin light chain (rMLC) is phosphorylated [12, 16, 17].

In this study we sought to examine myosin filament assembly using two well-known contractile stimuli: potassium chloride $(\mathrm{KCl})$ and acetylcholine $(\mathrm{ACh})$. Previous studies have shown that, when examined under EM, KCl-stimulated tissue has markedly fewer myosin filaments than ACh-stimulated tissue, despite producing similar force [24]. $\mathrm{KCl}$ activated muscle was also found to be more susceptible to mechanical perturbation than ACh activated muscle [39]. Based on these observations we used mechanical perturbation to examine the stability of the contractile units formed during $\mathrm{KCl}$ and $\mathrm{ACh}$ stimulation. We also sought to determine the role of rMLC-phosphorylation in filamentogenesis.

\subsection{Materials and Methods}

\subsubsection{Tissue Preparation and Equilibration}

Sheep tracheas were obtained from a local abattoir and prepared as previously described $[9,18]$. All experimental procedures were approved by the Animal Care Committee and Biosafety Committee of the University of British Columbia and conformed to the 
guidelines set out by the Canadian Council on Animal Care (Appendix A). Briefly, tracheas were transported and stored in $4{ }^{\circ} \mathrm{C}$ physiological saline solution (PSS). The PSS consisted of (mM): $118 \mathrm{NaCl}, 5 \mathrm{KCl}, 1.2 \mathrm{NaH}_{2} \mathrm{PO}_{4}, 22.5 \mathrm{NaHCO}_{3}, 2 \mathrm{MgSO}_{4}, 2 \mathrm{CaCl}_{2}$, and $2 \mathrm{~g} / \mathrm{l}$ dextrose. Experiments were performed within five days of obtaining the tissue. The in situ muscle length was determined from a 3/4" tracheal segment prior to removal of the $\mathrm{C}$-shaped cartilage and was used as a reference length $\left(\mathrm{L}_{\mathrm{ref}}\right)$ for normalization of all length measurements. After removal of the cartilage, the muscle bundles, held at $\mathrm{L}_{\text {ref, }}$ were dissected free of adventitial connective tissue and epithelium. Muscle strips measuring 1-1.5 mm wide, 0.2-0.3 $\mathrm{mm}$ thick, and $\sim 6 \mathrm{~mm}$ in length were removed and attached to custom-designed aluminum foil clips at each end. The muscle strips were then vertically mounted onto a custom-designed myograph capable of measuring tension and length change. The resonant frequency of the force transducer is approximately $500 \mathrm{~Hz}$ and the muscle lever is capable of making a $1 \mathrm{~mm}$ step in $\sim 1 \mathrm{~ms}$. While attached to the myograph, the muscle was entirely submerged in a tissue bath filled with warmed PSS $\left(37^{\circ} \mathrm{C}\right)$. An outer water-jacket maintained the temperature of the tissue bath at $37^{\circ} \mathrm{C}$.

The muscle strips held at $\mathrm{L}_{\text {ref }}$ were initially stimulated for 10 seconds with electrical field stimulation (EFS) at 5 minute intervals during a $1.5 \mathrm{~h}$ period of equilibration. EFS consisted of a $60 \mathrm{~Hz}$ alternating current at a voltage of $15-20 \mathrm{~V}$ which resulted in maximal force generation. PSS gassed with $5 \% \mathrm{CO}_{2} / 95 \% \mathrm{O}_{2}$ to maintain a $\mathrm{pH}$ of 7.4 was changed in the tissue bath at 5 minute intervals following contraction. The tissue was considered equilibrated when the muscle generated consistent maximal isometric force $\left(F_{\max }\right)$ with minimal resting tension (less than $1 \mathrm{mN}$ or $3 \%$ of $F_{\max }$ ). 


\subsubsection{Stimulation with Potassium Chloride or Acetylcholine}

Following equilibration the muscle was either stimulated with PSS containing $40 \mathrm{mM}$ $\mathrm{KCl}$ or acetylcholine (ACh). The $40 \mathrm{mM} \mathrm{KCl}$ PSS was composed of (mM): $83 \mathrm{NaCl}, 40$ $\mathrm{KCl}, 1.2 \mathrm{NaH}_{2} \mathrm{PO}_{4}, 22.5 \mathrm{NaHCO}_{3}, 2 \mathrm{MgSO}_{4}, 2 \mathrm{CaCl}_{2}$, and $2 \mathrm{~g} / \mathrm{l}$ dextrose; where $\mathrm{KCl}$ was increased according to an equimolar decrease in $\mathrm{NaCl}$. The $\mathrm{KCl}$ concentration was determined by using $10^{-6} \mathrm{M}$ atropine, a competitive antagonist of the muscarinic acetylcholine receptor (Sigma A0132: Sigma-Aldrich), as described below. Since KCl depolarizes the smooth muscle cell by altering the membrane potential it was critical to find a concentration at which the response to $\mathrm{KCl}$ was maximal and yet independent of neuronal release of ACh. The process used to determine this is described in Figure 3.1. First, a supramaximal $\mathrm{KCl}$ concentration $(80 \mathrm{mM})$ was used to stimulate the muscle (Fig. 3.1A), then the muscle was stimulated with varying concentrations of $\mathrm{KCl}$ (Fig. 3.1C). At the end of the protocol, the muscle was treated with $10^{-6} \mathrm{M}$ atropine (Fig. 3.1B), and the force developed in the presence of atropine was taken as the maximal force attributable to depolarization of the smooth muscle alone (the atropine was given at the end of the protocol since the treatment was not readily reversible). This response was verified by stimulation with EFS, which is known to stimulate smooth muscle via neuronal ACh release, since the force in response to EFS was completely abolished in the presence of atropine. $40 \mathrm{mM} \mathrm{KCl}$ was consistently determined as the concentration that minimized neuronal stimulation. This was confirmed in another muscle strip by observing that force was maintained at the level of $40 \mathrm{mM} \mathrm{KCl}$ stimulation even after treatment with atropine. The ACh concentration was determined as the concentration at which force response for a given muscle was equal to normalized force response of its pair to $40 \mathrm{mM} \mathrm{KCl}$, within 
the range of $10^{-7}$ and $10^{-3} \mathrm{M}$. For example, Muscle A produces $50 \mathrm{mN}$ with EFS while Muscle B produces $45 \mathrm{mN}$. Muscle A is stimulated with $\mathrm{KCl}$ and produces $60 \mathrm{mN}$, thus the matching concentration of $\mathrm{ACh}$ would produce $(60 / 50) \times 45$ or $54 \mathrm{mN}$ of force, so that each strip is producing $120 \%$ of EFS force in response to $\mathrm{KCl}$ or $\mathrm{ACh}$. Since the normalization depended on the maximal force response to EFS, the absolute force value produced by a pair was limited to $15 \%$ difference, otherwise the experiment would not proceed. Following stimulation by $40 \mathrm{mM} \mathrm{KCl}$ or a variable $\mathrm{ACh}$ concentration that produced the equivalent relative force, muscle strips were: 1) fixed for electron microscopy, 2) exposed to a period of length oscillation, or 3) flash frozen in liquid nitrogen for western blot analysis. Importantly, the muscle strips were not stimulated with both $\mathrm{KCl}$ and $\mathrm{ACh}$ at different time points, because it has been shown [39] that $\mathrm{ACh}$ sensitizes the muscle to the subsequent stimulations, even when the stimulation is $\mathrm{KCl}$ induced depolarization. Instead paired muscle strips from the same trachea were used and each strip was exposed to either $\mathrm{KCl}$ or $\mathrm{ACh}$ but not both. Paired strips from the same animal were used in one of the three procedures listed above, but not all the procedures. 

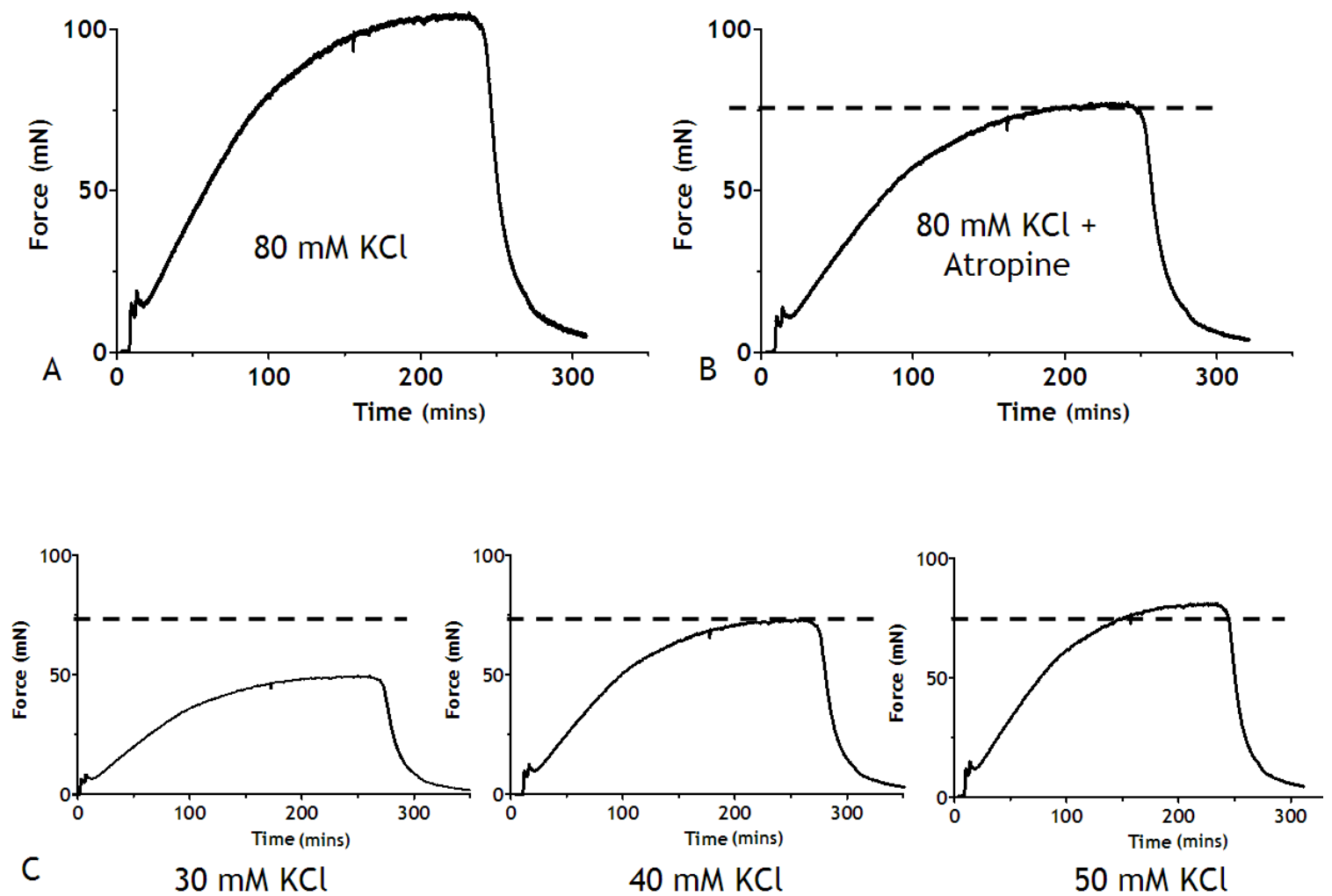

Figure 3.1 Determination of $\mathbf{K C l}$ concentration. To determine the concentration of $\mathrm{KCl}$ that elicits maximal force without involving neuronal stimulation, a supramaximal concentration of $\mathrm{KCl}$ was used as a stimulus (A). B) Applying $10^{-6} \mathrm{M}$ atropine to the muscle outlined the force contribution strictly due to muscle cell depolarization. C) Varying concentrations of $\mathrm{KCl}$ were used to match the force response recorded in $\mathrm{B}$. $40 \mathrm{mM} \mathrm{KCl}$ was determined as the maximal concentration.

\subsubsection{Electron Microscopy}

The protocol for EM fixation has been described previously [10, 19]. Briefly, after stimulation and reaching the force plateau the muscle, along with a force-matched pair, was fixed while it was still attached to the myograph so that the muscle was not physically disturbed and the force could be continuously monitored. The primary fixative 
was a cocktail consisting of: $1.5 \%$ paraformaldehyde, $1.5 \%$ glutaraldehyde, and $0.5 \%$ tannic acid in $0.1 \mathrm{M}$ sodium cacodylate solution warmed to $37^{\circ} \mathrm{C}$. After initial fixation the muscle was cut into 6-8 blocks and put in fixing solution on a shaker at $4^{\circ} \mathrm{C}$ for $2 \mathrm{~h}$. The blocks were then washed once with $0.1 \mathrm{M}$ sodium cacodylate and twice with $1.25 \%$ sodium bicarbonate for 10 min each. The blocks were processed in a secondary fixative of $1 \% \mathrm{OsO}_{4}-1.25 \% \mathrm{NaHCO}_{3}$ for $1.5 \mathrm{~h}$, followed by three washes in distilled water (30 min). En bloc staining was accomplished by placing the blocks in $1 \%$ uranyl acetate for 1 $\mathrm{h}$, followed by three washes with distilled water. The blocks were then dehydrated using increasing concentrations of ethanol $(50,70,80,90,95 \%$ - 10 min each; $100 \%-3 \times 15$ min each) followed by three washes with propylene oxide (15 min each). The blocks were gradually infiltrated overnight with $\mathrm{TAAB} 812$ resin mix and then embedded in molds and placed in an oven at $60^{\circ} \mathrm{C}$ overnight. The embedded blocks were sectioned on a microtome with a diamond knife and placed on copper mesh grids. The section thickness was $50 \mathrm{~nm}$. After sectioning the grids were stained with $1 \%$ uranyl acetate followed by Reynolds lead citrate for 4 and $3 \mathrm{~min}$, respectively. The images were obtained with a Technai transmission electron microscope (FEI Company: Hillsboro, OR).

\subsubsection{Length Oscillations}

At the force peak muscle strips stimulated with either $\mathrm{KCl}$ or $\mathrm{ACh}$ were mechanically perturbed by a fast, short duration length oscillation. The oscillation occurred at a frequency of $20 \mathrm{~Hz}$, amplitude of $10 \% \mathrm{~L}_{\mathrm{ref}}$, and duration of $2 \mathrm{sec}$. A short duration oscillation was chosen due to the fact that the force produced in response to $\mathrm{KCl}$ has a 
tendency to decline over time, compared to a more consistent response to ACh. Thus, the short duration minimizes any time-dependent changes in force during measurement. Length oscillations were analyzed as described below.

\subsubsection{Determining Myosin Light Chain Phosphorylation}

Myosin light chain phosphorylation was determined from a protocol adapted from previously described assays $[20,21]$. $\mathrm{KCl}$ - or ACh-stimulated muscle strips were flash frozen in liquid nitrogen. A separate group of muscle strips was equilibrated but not stimulated before freezing. Each trio of muscle strips (non-stimulated, $\mathrm{ACh}$, and $\mathrm{KCl}$ ) were removed from the same trachea. After freezing, the strip was removed from the myograph apparatus and placed in a pre-chilled acetone solution containing 5\% trichloroacetic acid (TCA) and $10 \mathrm{mM}$ dithiothreitol (DTT), then stored at $-80^{\circ} \mathrm{C}$. Immediately before assay the TCA-DTT-acetone solution was replaced with room temperature $10 \mathrm{mM}$ DTT-acetone, two washes. Afterwards the muscle was cut free of its aluminum foil clips and weighed to determine its mass. The muscle was then placed into a urea extraction buffer $(0.2 \mathrm{~mL} / \mathrm{mg})$ and homogenized. The extraction buffer consisted of: $0.1 \%$ SDS, $8 \mathrm{M}$ urea, $20 \mathrm{mM}$ Tris $\mathrm{HCl} \mathrm{pH}$ 7.7, 1 mM EDTA, $10 \mathrm{mM}$ EGTA, $22 \mathrm{mM}$ glycine, 10 mM DTT, 5X protease inhibitor cocktail (Sigma P8340: Sigma Aldrich), and 1X phosphatase inhibitor (Sigma P5726: Sigma Aldrich). The homogenate was left to macerate for $1 \mathrm{~h}$ on an inverter. The samples were then centrifuged at 14,000 $\mathrm{g}$ for 30 minutes at room temperature. After centrifugation the supernatant was harvested for further processing. 
Sample aliquots were added to a loading buffer consisting of $1 \mathrm{M}$ Tris $\mathrm{HCl} \mathrm{pH} 6.8$, SDS, Bromophenol Blue, glycerol, and water. The samples were boiled for 3 min before loading on to $10 \%$-SDS gels. SDS-PAGE was performed at $90 \mathrm{~V}$ in the stacking gel and $120 \mathrm{~V}$ in the resolving gel for $1 \mathrm{~h}$. The running buffer consisted of $(\mathrm{g} / \mathrm{L}): 72$ glycine, 15.1 Tris-base, and 5 SDS. Protein was transferred onto PVDF membrane using a transfer buffer consisting of: $192 \mathrm{mM}$ glycine, $25 \mathrm{mM}$ Tris-base, and 20\% methanol. Transfer occurred at $90 \mathrm{~V}$ for $90 \mathrm{~min}$. The membranes were then blocked with 5\% BSA-TBS$0.1 \%$ Tween for $1 \mathrm{~h}$ and after incubated with 1:1000 MLC-P primary antibody (Cell Signaling 3674S) at $4^{\circ} \mathrm{C}$ overnight. On the next day, after 3 washes for 10 min with TBS$\mathrm{T}$, the membrane was blotted with an anti-rabbit infrared secondary antibody for $1 \mathrm{~h}$ at room temperature. This blotting procedure was repeated using a 1:200 pan-MLC primary antibody (Santa Cruz sc-48414) to determine total MLC content and an anti-mouse infrared secondary antibody. Bands were quantified as described below.

\subsubsection{Morphometric and Statistical Analysis}

Electron micrograph images were taken from transverse sections of the tissue. Images of single cells (e.g. Fig 3.2 and 3.3) were randomly taken from the sections and a blinded counter manually enumerated myosin thick filaments and measured the cross-sectional area of the cell by manual tracing using Image Pro Plus 5 (Media Cybernetics: Bethesda, $\mathrm{MD})$. Three fixed muscle strips per group ( $\mathrm{KCl}$ or $\mathrm{ACh})$ were analyzed by taking 15 cell images per strip. 
Length oscillation data were analyzed by examining the resulting force peaks from each stretch cycle of the oscillation. The releases (troughs) were considered a less reliable measure of stiffness because the release creates slack in the force-transducer system. Therefore, the displacement of the muscle is only accurate during the stretch cycle.

Myosin light chain phosphorylation and total myosin light chain content were determined from western blot densitometry analysis using a LI-COR Odyssey Scanner (LI-COR Biosciences: Lincoln, NE). MLC-P was divided by total MLC content in each group. Paired (and trios of) muscle strips were force-matched according to the force generated by EFS. The force response to $40 \mathrm{mM} \mathrm{KCl}$ was used for normalization of force. Aggregate data were expressed as mean \pm SEM. Myosin filament density data, oscillation data, and myosin light chain phosphorylation were compared by ANOVA using GraphPad Prism 5 (GraphPad Software, Inc.: La Jolla, CA). $\mathrm{p} \leq 0.05$ was considered to be sufficient to reject the null hypothesis.

\subsection{Results}

\subsubsection{Force Response and Myosin Filament Density}

Stimulation with $40 \mathrm{mM} \mathrm{KCl}$ creates a consistent contraction that lasts for minutes, similar to stimulation with ACh (Fig. 3.2A). The force response to each stimulus was highly variable across animals but consistent across muscle strip pairs removed from the same trachea. Thus, the ACh concentration was not predetermined nor easily predicted.

Paired muscle strips producing similar $F_{\max }$ to EFS were force matched in response to 
$\mathrm{KCl}$ and $\mathrm{ACh}$ (Fig. 3.2B), then fixed for electron microscopy, mechanically perturbed, or frozen for western blot analysis.

Cross-sectional images of 15 different cells per muscle strip were taken from three pairs of muscle strips stimulated with either $\mathrm{KCl}$ or ACh (Fig. 3.3 and 3.4). Images taken of KCl-stimulated cells had noticeably fewer myosin thick filaments (Fig. 3.3) than AChstimulated cells (Fig. 3.4). All myosin thick filaments were counted and divided by the cell cross-sectional area to determine thick filament density (Fig. 3.5). For the $\mathrm{KCl}$ group thick filament density averaged $10.6 \pm 0.84$, ACh density averaged $36.6 \pm 2.8$ per $\mu \mathrm{m}^{2}$, the two groups were significantly different (ANOVA: $\mathrm{p}<0.0001)$. Cross-sectional area $\left(\mu \mathrm{m}^{2}\right)$ averaged $9.78 \pm 0.9$ for the $\mathrm{KCl}$ group and $8.99 \pm 0.7$ for the $\mathrm{ACh}$ group, not significantly different $(\mathrm{p}=0.293)$. 

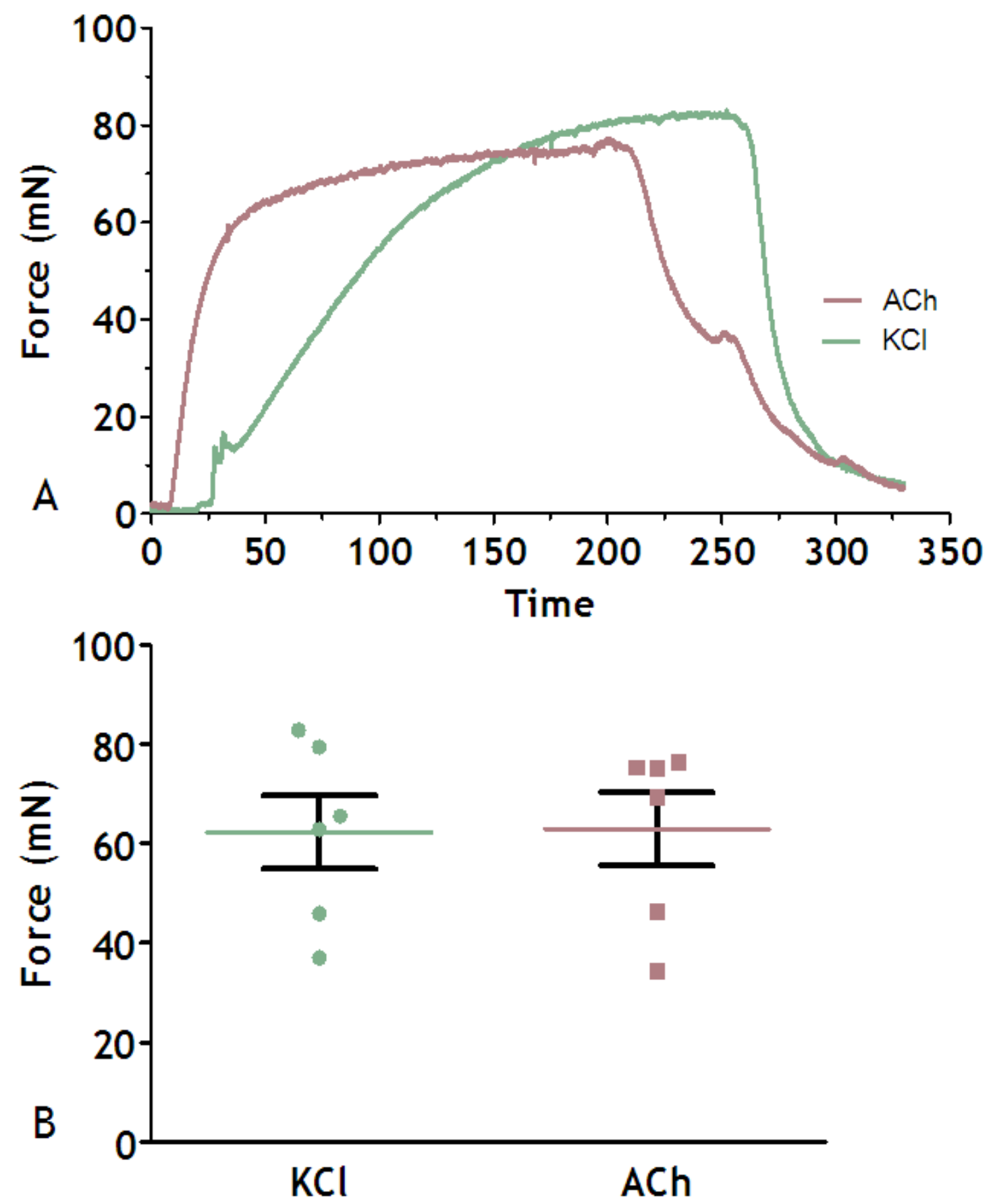

Figure 3.2 $\mathrm{KCl}$ and $\mathrm{ACh}$ force-matching. A) A sample trace demonstrating the contractile responses to $40 \mathrm{mM} \mathrm{KCl}$ and a force-matched concentration of $\mathrm{ACh}$. B) Comparison of force between the $\mathrm{KCl}$ and $\mathrm{ACh}$ groups prior to length oscillation. This comparison is representative of force prior to electron microscopy fixation and myosin 
light chain phosphorylation determination. Error bars indicate SEM with the center line indicating mean. $\mathrm{n}=6$ per group. Paired Student's t-test: $\mathrm{p}=0.893$.

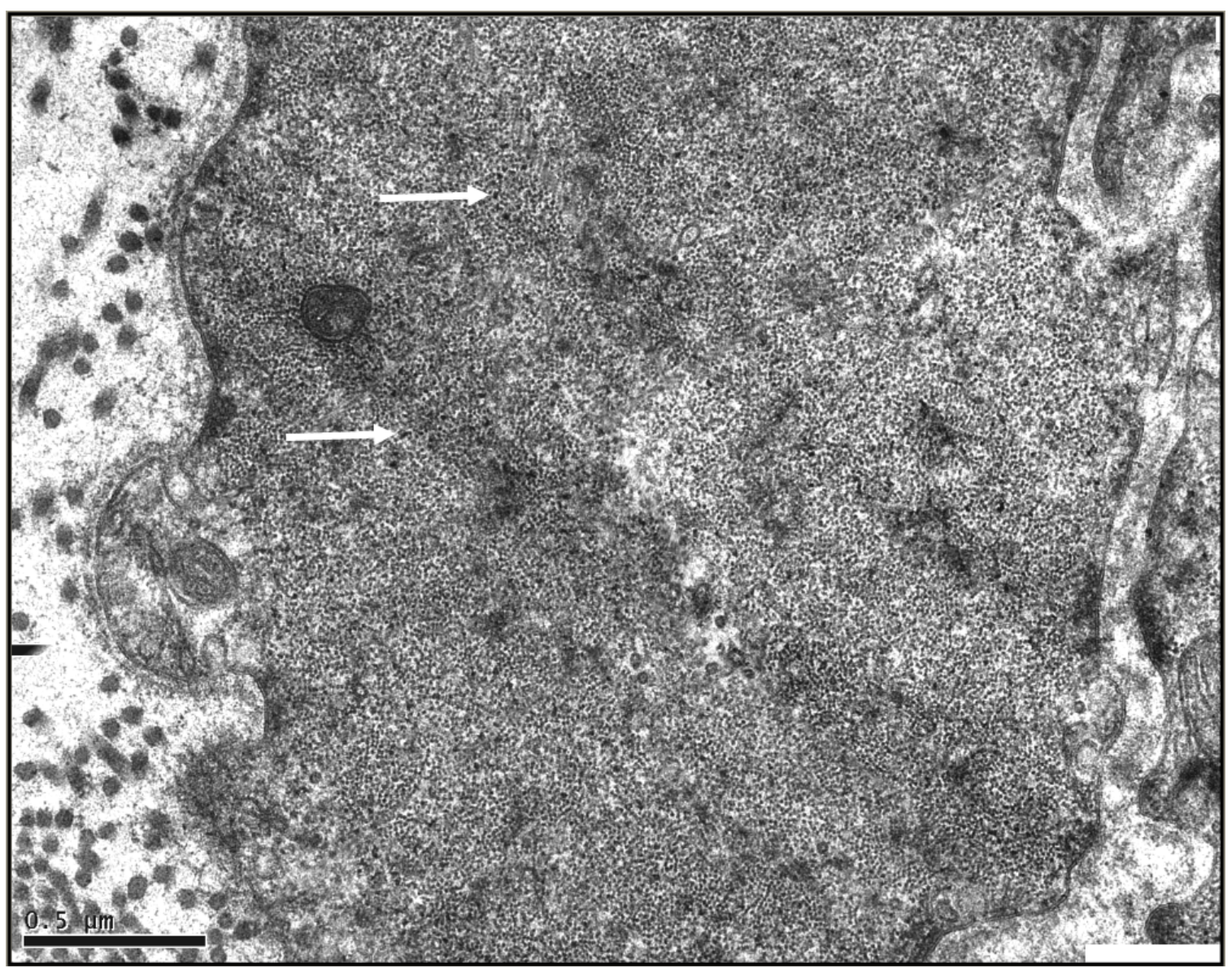

Figure 3.3 Cross-sectional electron micrograph of a $\mathrm{KCl}$ contracted cell. Traditional cellular structures are apparent. Actin filaments are present throughout the cell. Few myosin filaments (white arrows are present). 


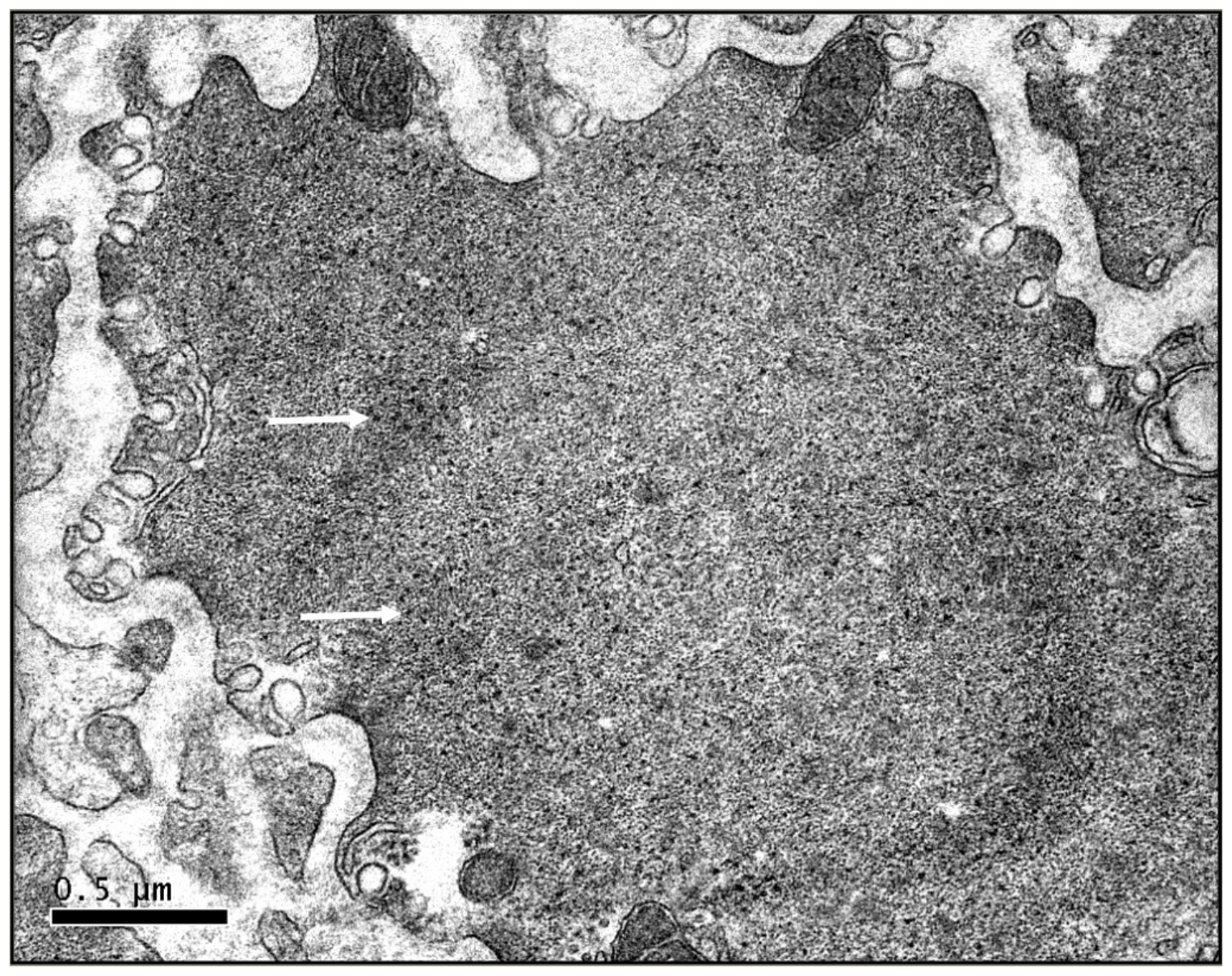

Figure 3.4 Cross-sectional electron micrograph of an ACh contracted cell. Unlike

$\mathrm{KCl}$ stimulated cells, the $\mathrm{ACh}$ contracted cell has many more myosin filaments (white arrows). 


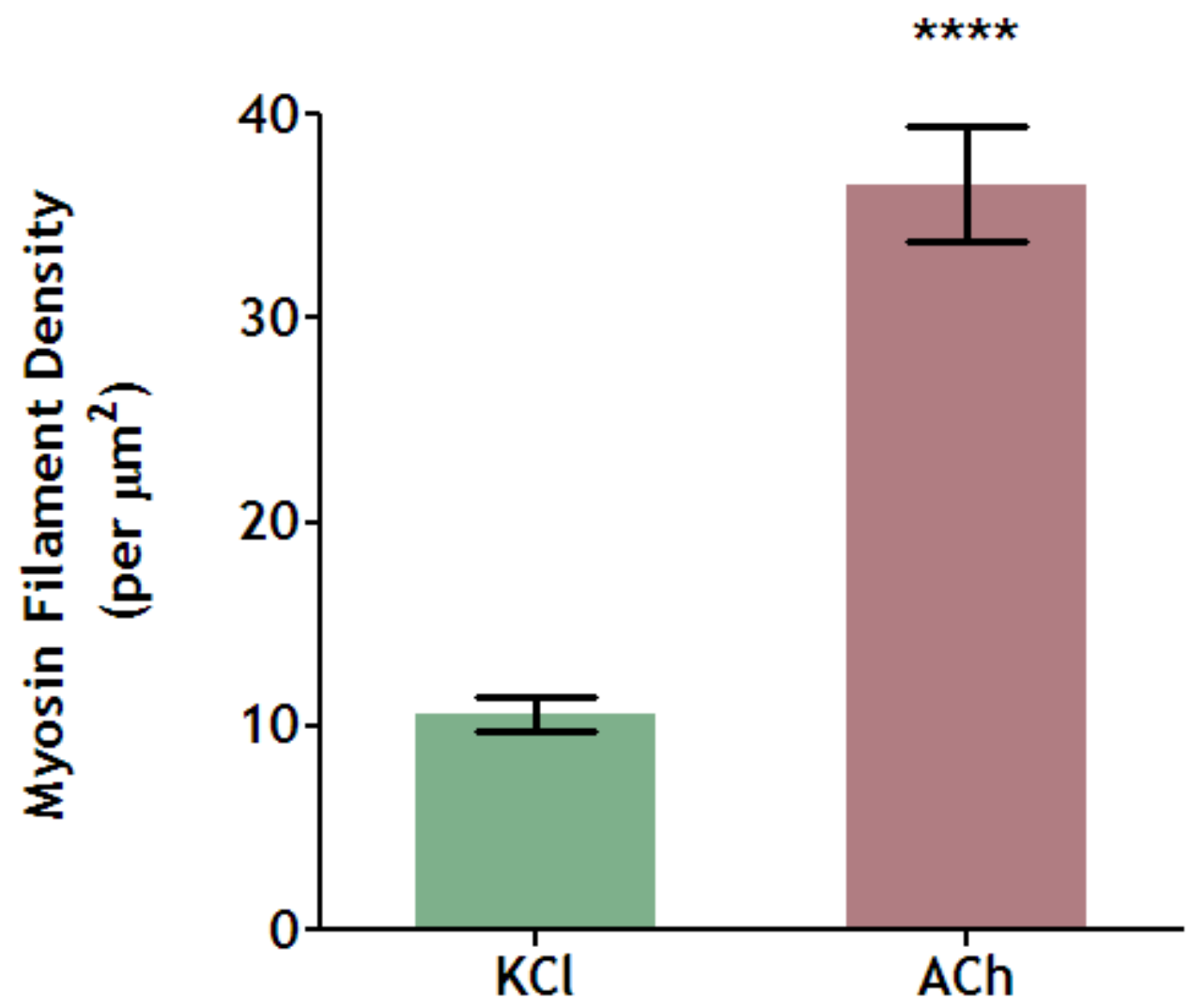

Figure 3.5 Myosin filament density. Myosin filament density determined from electron micrographs of KCl- and ACh-stimulated ASM cells (see Figs. 3.3 and 3.4). Myosin filaments were quantified in a sample of 15 different cells (per ASM strip) and divided by the total cross-sectional area $\left(\mu \mathrm{m}^{2}\right)$. ANOVA: ${ }^{* * *}=\mathrm{p}<0.0001, \mathrm{n}=3$ (number of ASM strips fixed). Error bars indicate SEM.

\subsubsection{Length Oscillation and Recovery}

Paired muscle strips ( $\mathrm{n}=6$ per group) were stimulated to contract after equilibration with either $40 \mathrm{mM} \mathrm{KCl}$ or the force-matched concentration of $\mathrm{ACh}$ (Fig. 3.6). At the force plateau a two-second length oscillation was applied followed by a recovery period. A 
representative force record of the length oscillations is shown in Figure 3.7. From the force record the peaks of the stretch cycle were extracted and replotted in Figure 3.8. The force response for the two groups were significantly different from one another (ANOVA: $\mathrm{p}<0.0001$ ). Although the first stretch of the oscillation for the $\mathrm{KCl}$-stimulated group had a higher peak force than the ACh-stimulated muscle (ANOVA: $p<0.05$ ) the peaks after the eleventh stretch were significantly lower in the $\mathrm{KCl}$-stimulated tissues than the comparable ACh peaks (Bonferroni post-tests: $\mathrm{p}<0.05$ ).

Following the length oscillation, the muscle strips, still exposed to $\mathrm{KCl}$ or $\mathrm{ACh}$, were allowed to recover (Fig. 3.9). Since the force of the ACh group declined less than the $\mathrm{KCl}$ group after oscillation, the $\mathrm{ACh}$ group recovered to a high force plateau than the $\mathrm{KCl}$ group. Neither group recovered its original force produced before oscillation (normalized force $=1$ ). The initial rate of force recovery and extent of recovery was greater in the $\mathrm{KCl}$-stimulated group (ANOVA: $\mathrm{p}<0.05$ ). This difference is expressed in the curvilinear regressions for each group $\left(\mathrm{KCl}\right.$ : force $=0.371+0.508 \mathrm{e}^{-0.056 t}$; ACh: force $=0.491+$ $0.427 \mathrm{e}^{-0.061 t}$, where force was normalized to the $40 \mathrm{mM} \mathrm{KCl}$ response and $t$ was time in seconds) and displayed more prominently when both curves are compared at the same starting force (Fig. 3.10). 


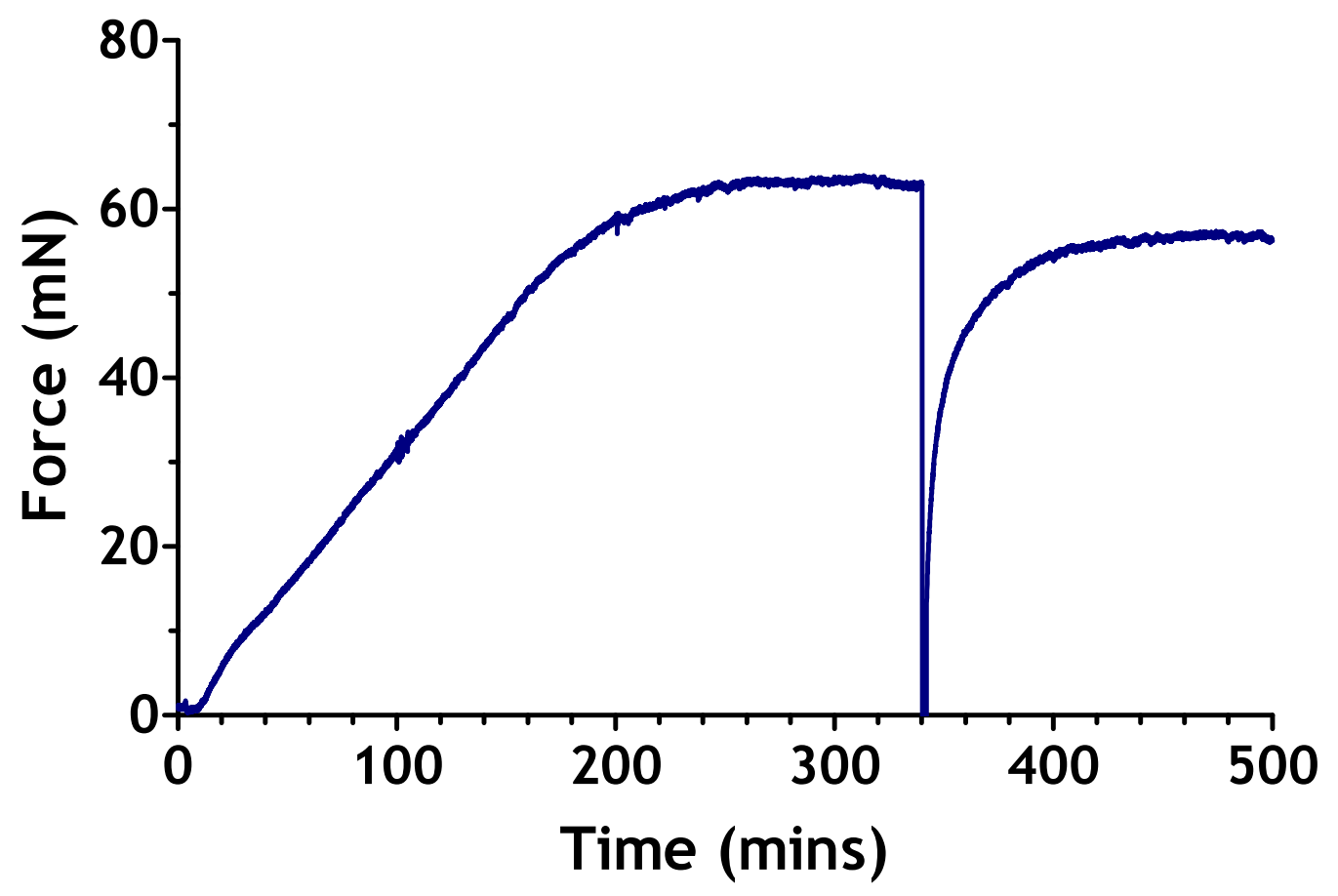

Figure 3.6 Sample force trace of an ASM strip with length oscillation and recovery. Muscle strips were stimulated to contract with either $\mathrm{KCl}$ or $\mathrm{ACh}$ and mechanically perturbed with a length oscillation at the force plateau. The muscle was allowed to recover following oscillation. 


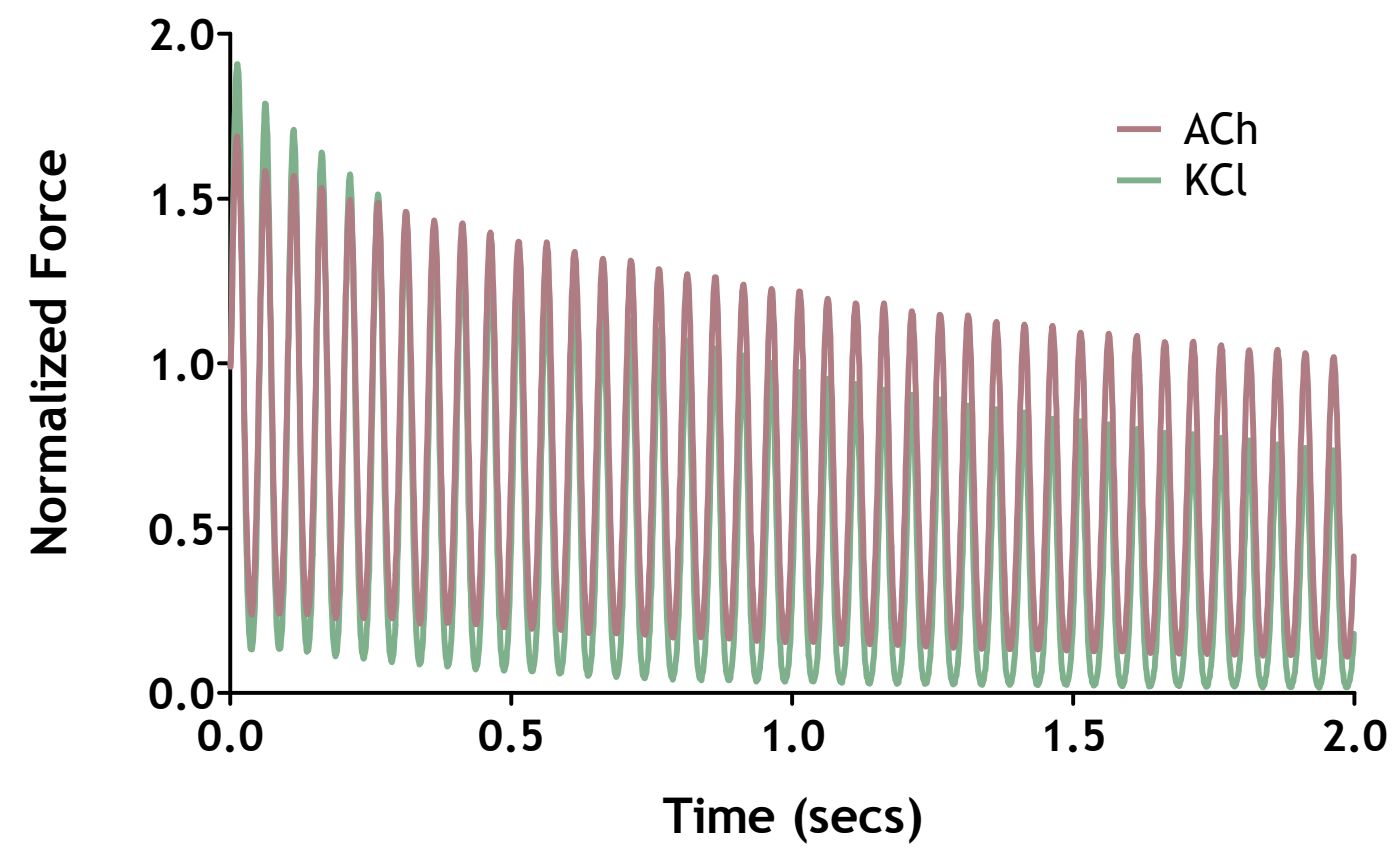

Figure 3.7 Length oscillation. Representative force trace of the length oscillation. ACh is represented in pink and $\mathrm{KCl}$ in green. The oscillation occurred at $20 \mathrm{~Hz}$ for $2 \mathrm{sec}$ at an amplitude of $10 \% \mathrm{~L}_{\text {ref. }}$ 


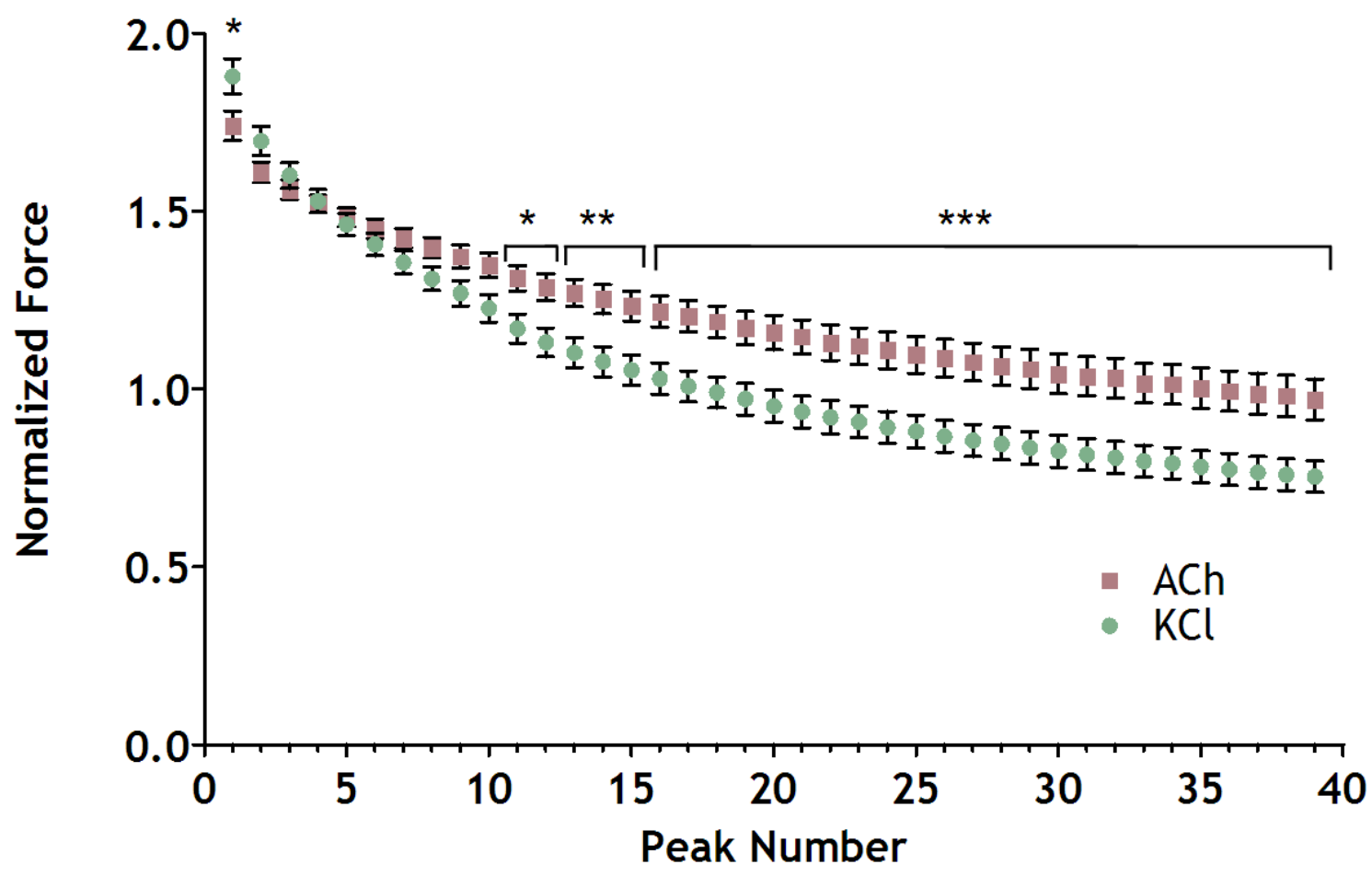

Figure 3.8 Force peaks from the stretch cycle of the length oscillation. ACh is represented in pink and $\mathrm{KCl}$ in green. Force was normalized to the response to $40 \mathrm{mM}$ KCl. $\mathrm{n}=6$ per group. ANOVA: ${ }^{*}=\mathrm{p}<0.05,{ }^{* *}=\mathrm{p}<0.01,{ }^{* * *}=\mathrm{p}<0.001$. Error bars indicate SEM. 


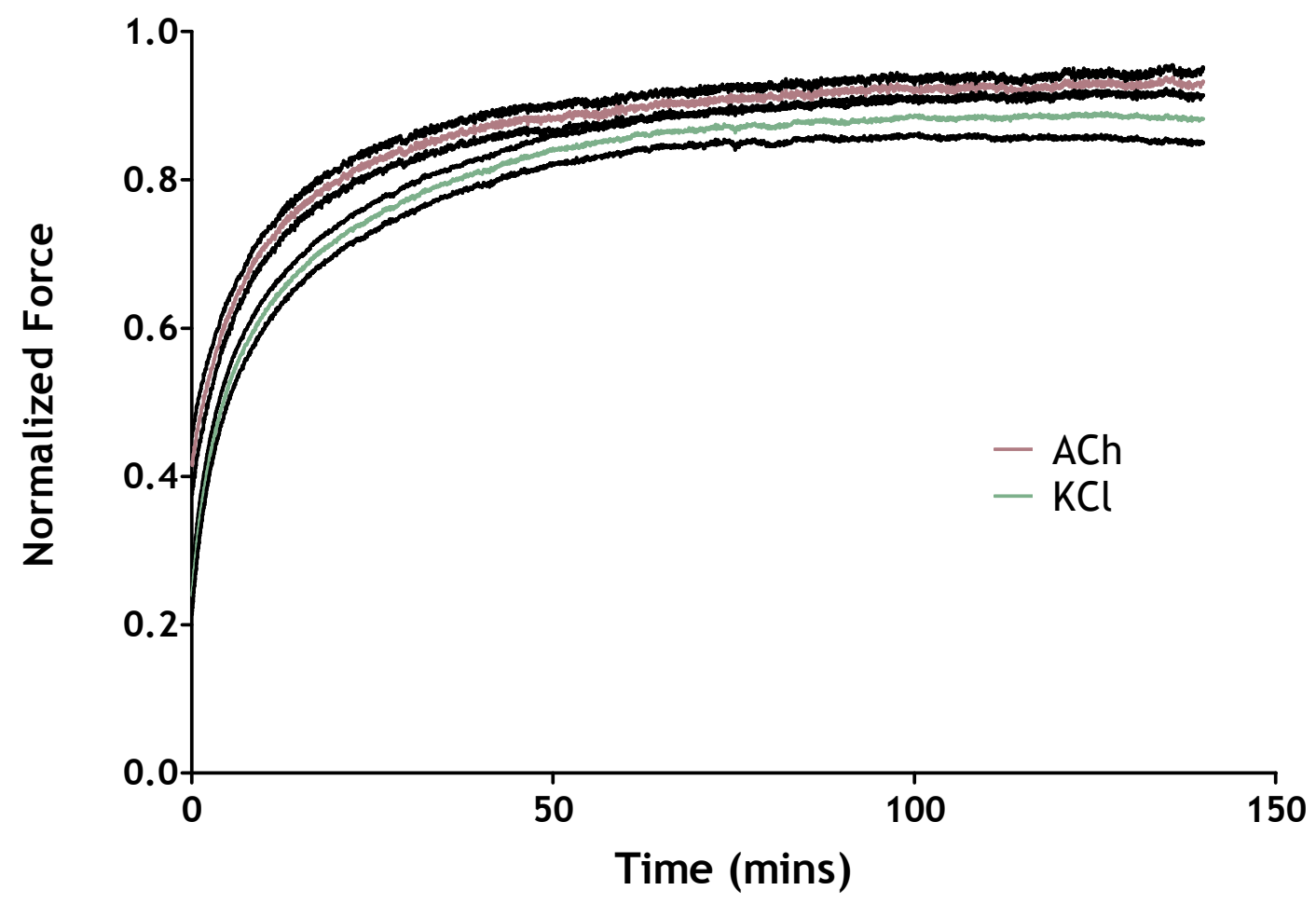

Figure 3.9 Recovery after length oscillation. Recovery of isometric force following length oscillation. $\mathrm{ACh}$ is represented in pink and $\mathrm{KCl}$ in green. $\mathrm{n}=6$ per group, with the upper and lower lines indicating SEM. 


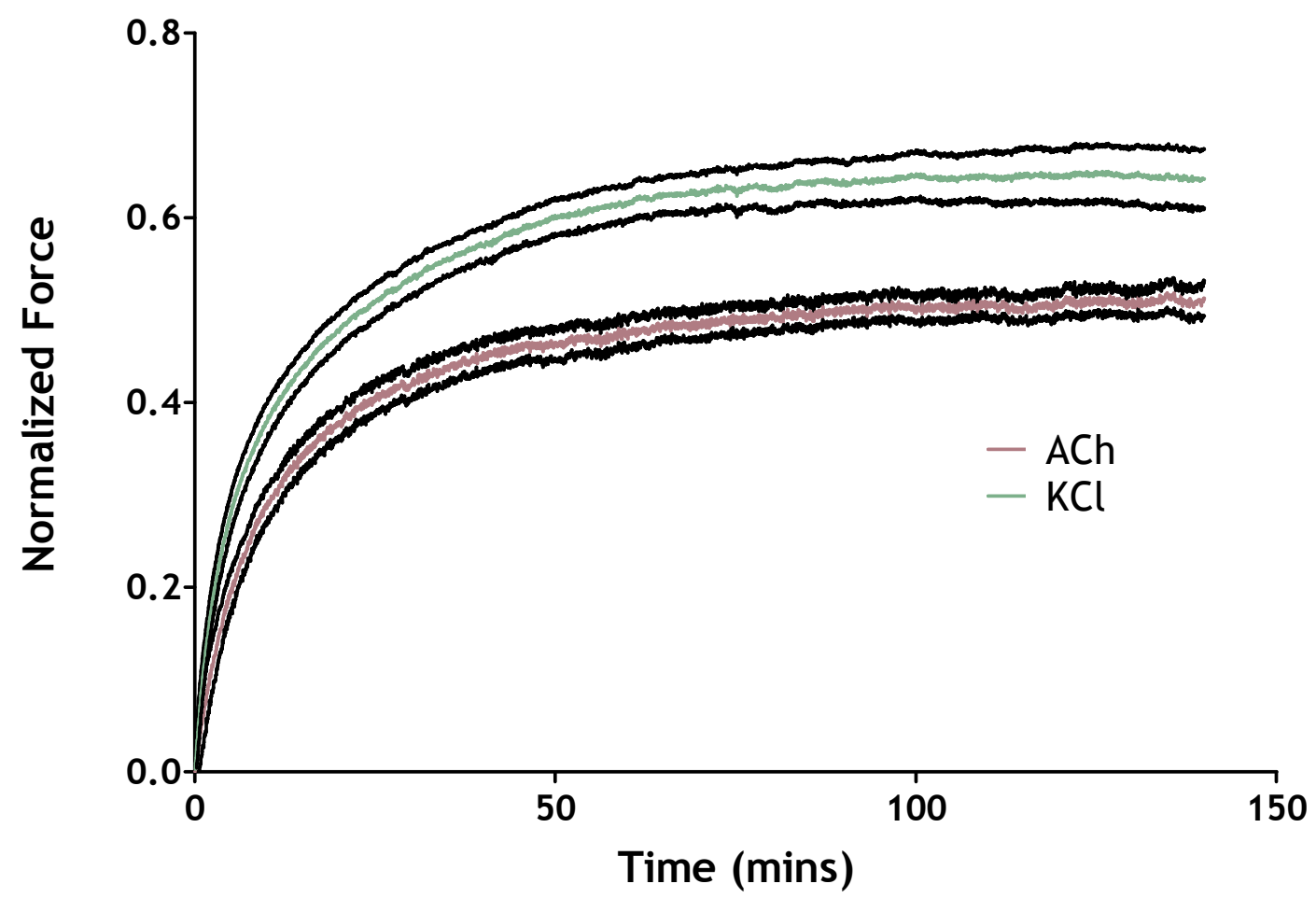

Figure 3.10 Adjusted recovery after length oscillation. Recovery of isometric force following length oscillation adjusted such that both $\mathrm{KCl}$ and $\mathrm{ACh}$ begin at zero force. $\mathrm{ACh}$ is represented in pink and $\mathrm{KCl}$ in green. $\mathrm{n}=6$ per group, with the upper and lower lines indicating SEM.

\subsubsection{Measurement of rMLC Phosphorylation}

Three groups of muscle strips ( $n=7$ per group) were flash frozen after being equilibrated and 1) not stimulated, 2) stimulated with $\mathrm{KCl}$, or 3) stimulated with ACh. The tissues were analyzed for rMLC phosphorylation and total rMLC content by western blot (Fig. 3.11). The ratio of phosphorylated rMLC expression to total rMLC content was compared among the groups. The $\mathrm{ACh}$ and $\mathrm{KCl}$ groups had significantly higher expression of 
phosphorylated rMLC compared to the unstimulated group (ANOVA: $\mathrm{p}=0.05$ ), while the $\mathrm{ACh}$ and $\mathrm{KCl}$ groups were not significantly different from one another.

\section{MLC-P}

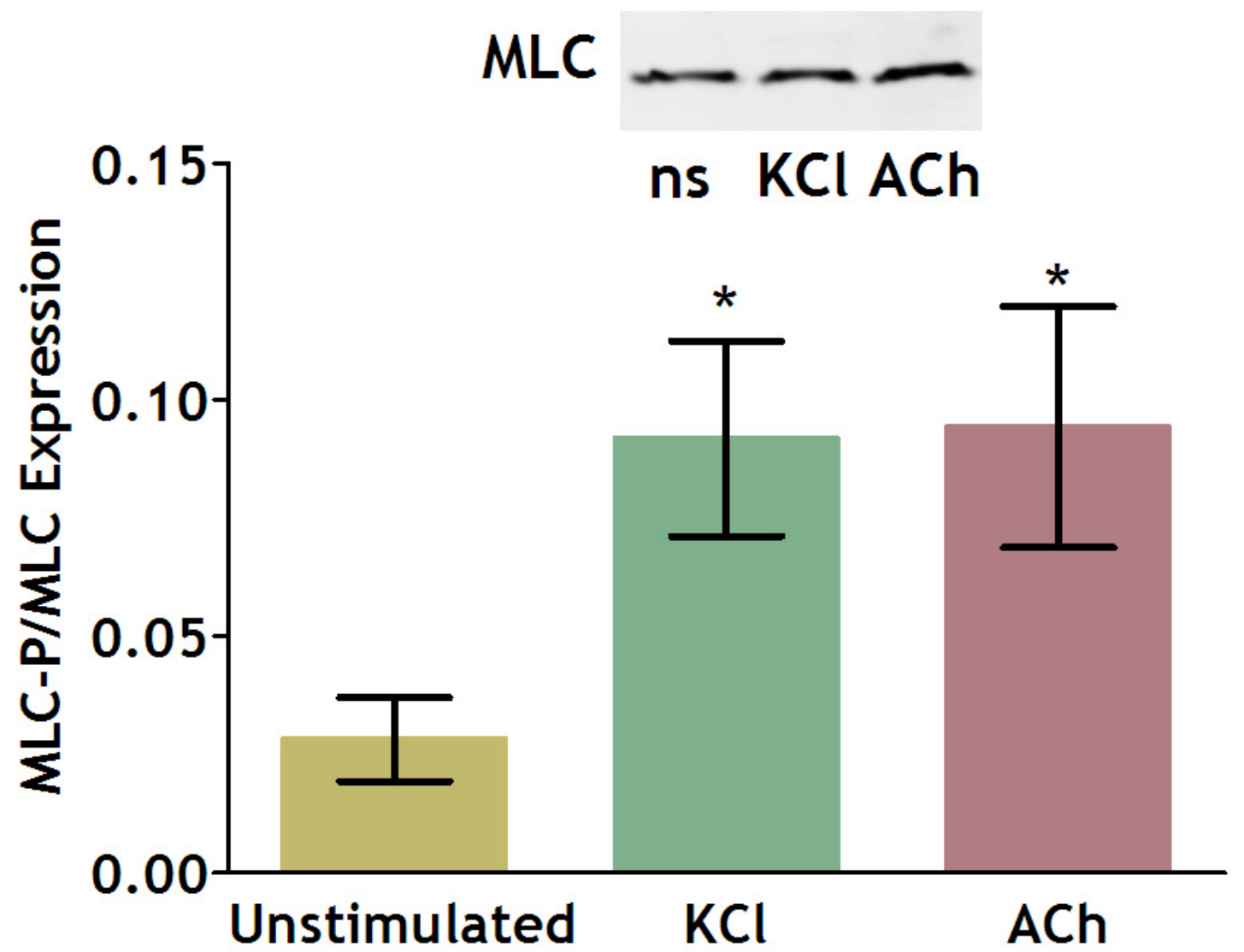

Figure 3.11 Myosin light chain phosphorylation. Expression of rMLC phosphorylation divided by total rMLC content. Muscle strips were frozen at the same time point as length oscillation and electron microscopy. Unstimulated ASM is represented in yellow, $\mathrm{KCl}$ in green, and $\mathrm{ACh}$ in pink. Error bars indicate SEM. $\mathrm{n}=7$ per group. ANOVA: ${ }^{*}=\mathrm{p}<0.05$, compared to unstimulated group. $\mathrm{KCl}$ and $\mathrm{ACh} \mathrm{rMLC}$ phosphorylation were not significantly different. $n s=$ non-stimulated in the representative western blot inset. 


\subsection{Discussion}

Potassium chloride is a frequently used contractile agonist in smooth muscle mechanical studies despite the fact that its mechanism of activation is not well understood. It is thought that $\mathrm{KCl}$ depolarizes the cellular membrane through the action of voltage-gated channels, leading to calcium release from the sarcoplasmic reticulum (SR) that is independent of inositol triphosphate $\left(\mathrm{IP}_{3}\right)$ release $[22,23]$. In contrast, acetylcholine activation is much better understood and thought to work through the canonical muscarinic G-protein coupled receptor-IP ${ }_{3}$ activation pathway [22]. ACh-stimulated smooth muscle cells have been shown to have an increased density of myosin filaments compared to unstimulated muscle [10], however, the precise mechanism through which filamentogenesis occurs has yet to be elucidated. In this study, we present an intriguing model to examine myosin filamentogenesis, in which both agonists produce strong contractions of equivalent force, but where one agonist, $\mathrm{KCl}$, does so without an increase in myosin filaments.

Both $\mathrm{ACh}$ and $\mathrm{KCl}$ cause a contraction with an initial rapid increase in force (phasic phase) followed by a prolonged tonic phase (Fig. 3.2A). The rate of rise in force was generally much slower with $\mathrm{KCl}$-stimulation. This could be due to a slower activation of cells since the release of $\mathrm{Ca}^{2+}$ from the SR relies on depolarization of the membrane and a sufficient influx of $\mathrm{Ca}^{2+}$ to trigger calcium-induced calcium release from the ryanodine receptors (RyR), independent of $\mathrm{IP}_{3}$-receptor mediated release. In comparison, ACh binds to the muscarinic receptor leading to $\mathrm{IP}_{3}$ release and its subsequent binding to the $\mathrm{IP}_{3}$

receptor and $\mathrm{Ca}^{2+}$ release, as well as RyR activation by $\mathrm{Ca}^{2+}$ induction and the $\mathrm{CD} 38$ - 
ADP-ribose pathway. These signal transduction pathways are likely to mobilize $\mathrm{SR} \mathrm{Ca}^{2+}$ release more rapidly than the diffusion-limited response to $\mathrm{KCl}$ activation. These differences are mirrored in the calcium release patterns of the two spasmogens seen in isolated ASM cells [23]. ACh-stimulation induces calcium waves that occur at a relatively high frequency and fast velocity, whereas, $\mathrm{KCl}$-stimulation induces minor localized calcium events at regular intervals followed by long duration, slowly propagating $\mathrm{Ca}^{2+}$ waves. Since these calcium waves in $\mathrm{KCl}$-stimulated smooth muscle are abolished by treatment with caffeine, a RyR activator, and clopiazonic acid, an inhibitor of the SR $\mathrm{Ca}^{2+}$ ATPase (SERCA), the $\mathrm{IP}_{3}$ activation pathway and influx of external $\mathrm{Ca}^{2+}$ likely play a minimal role in $\mathrm{KCl}$ force generation [23]. These differences in calcium signaling could also explain the instability of the tonic force produced by $\mathrm{KCl}$ over periods longer than five minutes, as the force tends to drift lower and then gradually increases again, whereas, the force response to $\mathrm{ACh}$ is very consistent over long periods and often gradually increases over time.

In this study we have demonstrated that $\mathrm{KCl}$-stimulated muscle has far fewer myosin filaments (Fig. 3.5), despite producing similar force compared to ACh-stimulated muscle (Fig. 3.2B). This is not the first study to identify this phenomenon. Ohashi and Nonomura [24] studied the effects of high $\mathrm{K}^{+}$solution on smooth muscle ultrastructure after a 30 minute exposure. They concluded that myosin filaments disappeared due to a mechanism of cell swelling, since they activated the tissue with a high $\mathrm{K}^{+}$Locke solution and noticed substantial cell swelling under EM. They argued that $\mathrm{Mg}^{2+}$, a critical ion in maintenance of myosin filaments, was being diluted in the cells, since Locke solution does not contain 
$\mathrm{Mg}^{2+}$. Upon addition of $\mathrm{Mg}^{2+}$ to $\mathrm{K}^{+}$-Locke solution they observed a recovery in filaments. Interestingly, our PSS contains $\mathrm{MgSO}_{4}$ and the duration of our contractions was always less than five minutes, reducing the possibility of cell swelling. We did not see the cell bloating that Ohashi and Nonomura observed [24], and the cell cross-sectional area of individual cells was not different in the $\mathrm{KCl}$ and $\mathrm{ACh}$ treated tissues. While we cannot entirely exclude the possible osmotic effects of ionic influx, it is likely that the lack of filaments is due to differences in cell signaling between the two stimuli. Regardless, it is clear that the magnitude of force produced by ASM cells does not correlate with the amount of myosin filaments formed during contraction stimulated by different modes of activation.

Based on this observation, we propose that force can be produced by myosin dimers or tetramers that act as ratchets capable of pulling actin filaments with opposite polarities in opposite direction. Since the myosin dimers or tetramers are not visible under EM, they can contribute to force generation but cannot be measured by filament counting on electron micrographs. We further hypothesize that without being in the filamentous form, the myosin dimers or tetramers are less able to maintain force during a large stretch, and are more likely to disengage their interaction with the actin filaments. This scheme is shown in Figure 3.12, in which the "sarcomere" under $\mathrm{KCl}$ activation is thought to consist of myosin dimers or tetramers that produce force but are in a filamentous conformation that cannot be resolved by EM. ACh activation, on the hand, is represented by the traditional contractile unit in which myosin dimers are connected into a filament that is visible under EM. Importantly, both conformations of the contractile unit have the 
same number of myosin crossbridges, thus they are able to produce the same force. By applying a length oscillation, the myosin filament in ACh activation may break but most of the dimers or tetramers would still be connected in a fragmented filament, whereas, the non-filamentous myosin would be less likely to maintain proper positioning in the actin filament lattice since the individual myosin dimers/tetramers are not attached to one another and only bound transiently through their crossbridge attachments to actin. The response to length oscillation (Fig. 3.8), supports this model, since the ACh-stimulated tissue maintains force better than the $\mathrm{KCl}$-stimulated tissue. It is unclear, however, why the $\mathrm{KCl}$-stimulated tissue has a higher force at the initial stretch compared to the AChstimulated tissue. Since the force can be taken as an index of stiffness because the displacement is the same between the two groups, it would suggest that the $\mathrm{KCl}$ stimulated cells are initially stiffer. It is possible that the $\mathrm{KCl}$-stimulation results in increased actin cytoskeletal stiffness [25] as has been demonstrated with optical magnetic twisting cytometry in cultured ASM cells. However, it is probably more likely that the difference is due to the activation of a greater proportion of slower cycling myosin crossbridges with $\mathrm{KCl}$-stimulation than ACh-stimulation, as has been demonstrated in vascular smooth muscle cells [26, 27], and also as evidenced by the slower rate of force development in $\mathrm{KCl}$-stimulated contraction (Fig. 3.2). Given the slower cycling rate, it is possible that more myosin crossbridges are attached to actin at the instant of the first stretch, thus increasing the perceived stiffness of the KCl-stimulated cells. 

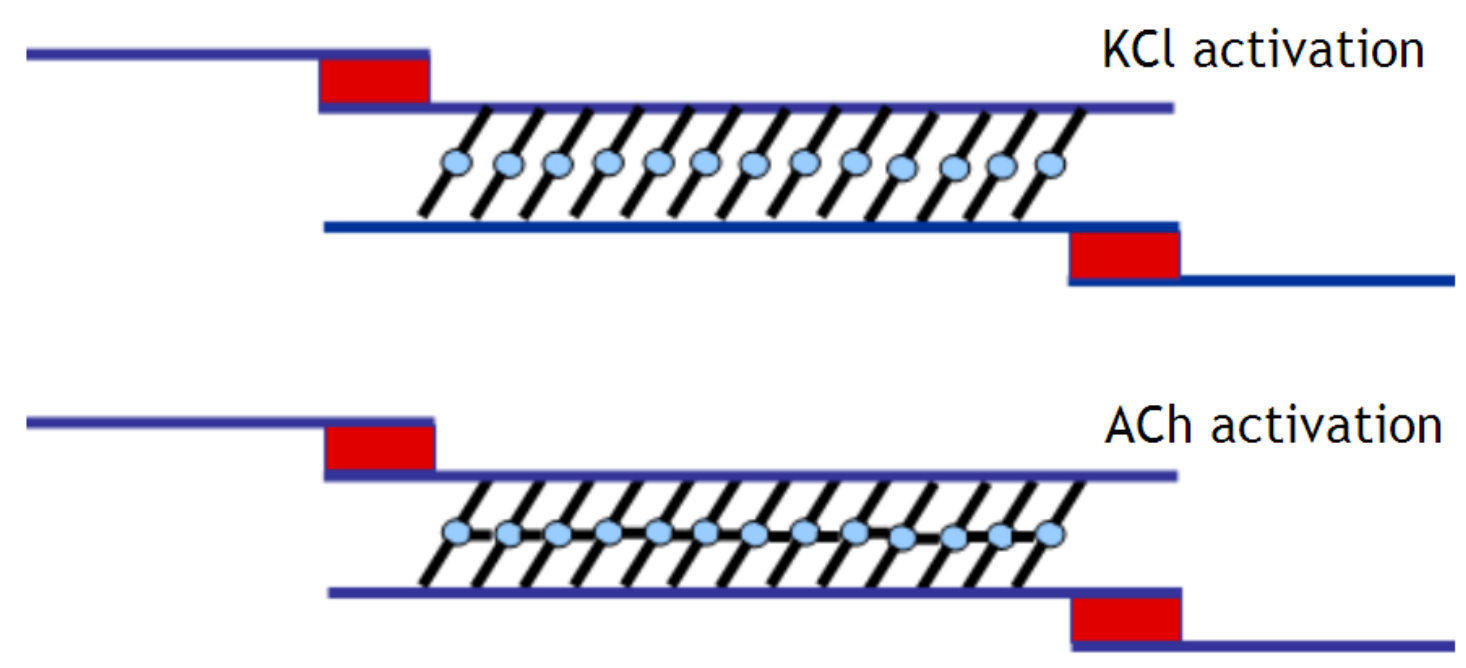

Figure 3.12 Contractile apparatus model of $\mathrm{KCl}$ and $\mathrm{ACh}$ activation. Model contractile apparatus representing an interpretation of the presented data. In $\mathrm{KCl}$ activation the blue actin filaments, attached to red Z-disk equivalents, flank myosin dimers which are not in a filamentous conformation. In ACh activation the myosin dimers are bound to each other into a filamentous conformation. This filamentous conformation may increase the stability of the contractile apparatus to mechanical perturbation, while the non-filamentous conformation may allow for faster force recovery (see text for details).

Examining the recovery of force after the oscillation (Fig. 3.9 and 3.10) provides additional support for our model of the smooth muscle contractile unit (Fig. 3.12). From Figure 3.10 it is clear that the magnitude of recovery is greater in the $\mathrm{KCl}$-stimulated tissue which is due to the large decline in force after oscillation. It is intriguing that both groups recover to the same relative force (Fig. 3.9) and are incapable of fully recovering force (1.0 normalized force, apparent in Fig. 3.6). Length oscillation causes disruption of the contractile filaments [28], but during recovery actin and myosin filaments (or myosin 
dimers/tetramers) are recruited into contractile units and contribute to force recovery [11, $28,29]$. The inability of the muscle to recover to its pre-oscillation force level suggests that the oscillation permanently disrupts a portion of contractile elements that cannot recover within the given time period. Recovery of these elements likely requires 15-30 minutes as is seen during the force response to EFS after the oscillation (see Fig. 1.2). Since the force recovers with time it is unlikely that the oscillation does any permanent damage to the muscle. From our models of $\mathrm{KCl}$ and $\mathrm{ACh}$ activation (Fig. 3.12), the oscillation would decrease force by disrupting actin filaments, but also by breaking myosin filaments in ACh-activation and dislocating myosin dimers/tetramers in $\mathrm{KCl}$ activation. The increased rate of force recovery in the KCl-stimulated tissue (Fig. 3.9 and 3.10) would support this view since it would be quicker for myosin dimers/tetramers to attach to intact actin filaments and contribute to force than it would be for fragments of myosin filaments of different lengths to fit back into the actin filament lattice that still have myosin fragments producing force.

This phenomenon of recruiting myosin into contractile units has been well characterized $[10,28,30]$, however, the mechanism is entirely unknown. It has been suggested that phosphorylation of the regulatory myosin light chain on the myosin molecule alters the conformation of the myosin molecule promoting filament formation [7, 12, 16, 31]. Further, rMLC phosphorylation has been shown to be associated with increased filament stability and resistance to mechanical perturbation [17]. In this study we had one-time point of rMLC phosphorylation in which there was no difference between $\mathrm{KCl}$ and $\mathrm{ACh}$ stimulated tissue (Fig. 3.11). Since KCl-stimulated muscle has fewer myosin filaments, it 
is unlikely that rMLC phosphorylation alone is responsible for the formation of myosin filaments. This lack of filaments made it impossible to comment on the role of rMLC phosphorylation on filament strength. Further biochemical analysis is needed to determine the importance of differential signaling cascades in myosin filamentogenesis.

Besides the $\mathrm{Ca}^{2+}$ mobilization differences mentioned previously, $\mathrm{ACh}$ stimulation also triggers the production of diacylglycerol (DAG). Since DAG activates PKCs, pathways downstream of PKCs are likely contributors to the differences between KCl-stimulation and ACh-stimulation, and may be where the differences in myosin filamentogenesis lie. PKCs influence rMLC phosphorylation through CPI-17 [32, 33], leading to calcium sensitization of force, and activate Raf which leads to ERK1/2 activation. ERK1/2 has been shown to have direct effects on caldesmon and tropomyosin, which could have roles in stabilizing the contractile apparatus [34-36]. Alternatively, ACh-stimulation may stabilize and lengthen actin filaments through p38 MAPK activation which leads to activation of HSP27 which is a known actin filament capping protein [37, 38].

In conclusion, we have demonstrated that KCl-stimulated ASM cells have fewer myosin filaments than ACh-stimulated cells, despite producing similar force. Mechanical experiments support the concept that smooth muscle cells are capable of producing force without myosin filaments. While different classes of myosin are known to produce force in a non-filamentous form, myosin II which is considered the force-producing myosin of muscle cells, is generally thought to only generate force in a filamentous form. We suggest that this mechanism is present in smooth muscle cells and may be an important 
contributor to proper functioning of the contractile apparatus that needs to maintain a high degree of structural malleability. This malleability is apparent when comparing the filament density of $\mathrm{KCl}$ stimulated muscle (Fig. 3.5) with the filament density of unstimulated muscle (e.g. in Fig. 2.10). Since $\mathrm{KCl}$ activated muscle has fewer myosin filaments than unstimulated muscle, it would imply a mechanism of filament disassembly immediately following contractile activation which in ACh activated muscle leads to filamentogenesis, but not in $\mathrm{KCl}$ activated muscle. This suggests that a large proportion of the filaments seen in relaxed smooth muscle cells are disassembled during the activation process. 


\subsection{References}

1. Mead J. Point: airway smooth muscle is useful. J Appl Physiol 2007;102:1708-9; discussion 1710 .

2. Fredberg JJ. Counterpoint: airway smooth muscle is not useful. J Appl Physiol 2007;102:1709-10; discussion 1710-1.

3. Huxley AF, Niedergerke R. Structural changes in muscle during contraction; interference microscopy of living muscle fibres. Nature 1954;173:971-973.

4. Huxley H, Hanson J. Changes in the cross-striations of muscle during contraction and stretch and their structural interpretation. Nature 1954;173:973-976.

5. Huxley AF. Muscle structure and theories of contraction. Prog Biophys Biophys Chem 1957;7:255-318.

6. Craig R, Woodhead JL. Structure and function of myosin filaments. Curr Opin Struct Biol 2006;16:204-212.

7. Craig R, Megerman J. Assembly of smooth muscle myosin into side-polar filaments. $J$ Cell Biol 1977;75:990-996.

8. Xu JQ, Harder BA, Uman P, Craig R. Myosin filament structure in vertebrate smooth muscle. J Cell Biol 1996;134:53-66.

9. Herrera AM, McParland BE, Bienkowska A, Tait R, Pare PD, Seow CY. 'Sarcomeres' of smooth muscle: functional characteristics and ultrastructural evidence. J Cell Sci 2005;118:2381-2392.

10. Herrera AM, Martinez EC, Seow CY. Electron microscopic study of actin polymerization in airway smooth muscle. Am J Physiol Lung Cell Mol Physiol 2004;286:L1161-8.

11. Mehta D, Gunst SJ. Actin polymerization stimulated by contractile activation regulates force development in canine tracheal smooth muscle. $J$ Physiol 1999;519 Pt $3: 829-840$.

12. Seow CY. Myosin filament assembly in an ever-changing myofilament lattice of smooth muscle. Am J Physiol Cell Physiol 2005;289:C1363-8.

13. Ford LE, Seow CY, Pratusevich VR. Plasticity in smooth muscle, a hypothesis. Can J Physiol Pharmacol 1994;72:1320-1324. 
14. Smolensky AV, Ragozzino J, Gilbert SH, Seow CY, Ford LE. Length-dependent filament formation assessed from birefringence increases during activation of porcine tracheal muscle. J Physiol 2005;563:517-527.

15. Smolensky AV, Ford LE. Filament lattice changes in smooth muscle assessed using birefringence. Can J Physiol Pharmacol 2005;83:933-940.

16. Trybus KM, Lowey S. Assembly of smooth muscle myosin minifilaments: effects of phosphorylation and nucleotide binding. J Cell Biol 1987;105:3007-3019.

17. Ip K, Sobieszek A, Solomon D, Jiao Y, Pare PD, Seow CY. Physical integrity of smooth muscle myosin filaments is enhanced by phosphorylation of the regulatory myosin light chain. Cell Physiol Biochem 2007;20:649-658.

18. Bosse Y, Chin LY, Pare PD, Seow CY. Adaptation of Airway Smooth Muscle to Basal Tone: Relevance to Airway Hyperresponsiveness. Am J Respir Cell Mol Biol 2008.

19. Chin LY, Bosse Y, Jiao Y, Solomon D, Hackett TL, Pare PD, Seow CY. Human airway smooth muscle is structurally and mechanically similar to that of other species. Eur Respir J 2009.

20. Mitchell RW, Seow CY, Burdyga T, Maass-Moreno R, Pratusevich VR, Ragozzino J, Ford LE. Relationship between myosin phosphorylation and contractile capability of canine airway smooth muscle. $J$ Appl Physiol 2001;90:2460-2465.

21. Gil FR, Zitouni NB, Azoulay E, Maghni K, Lauzon AM. Smooth muscle myosin isoform expression and LC20 phosphorylation in innate rat airway hyperresponsiveness. Am J Physiol Lung Cell Mol Physiol 2006;291:L932-40.

22. Dowell ML, Lavoie TL, Lakser OJ, Dulin NO, Fredberg JJ, Gerthoffer WT, Seow $\mathrm{CY}$, Mitchell RW, Solway J. MEK modulates force fluctuation-induced relengthening of canine tracheal smooth muscle. Eur Respir J 2010.

23. Perez JF, Sanderson MJ. The frequency of calcium oscillations induced by 5-HT, $\mathrm{ACH}$, and $\mathrm{KCl}$ determine the contraction of smooth muscle cells of intrapulmonary bronchioles. J Gen Physiol 2005;125:535-553.

24. Ohashi . Disappearance of myofilaments during isometric potassium contraction of smooth-muscle. Biomedical research [serial online]; vol. 5.

25. Deng L, Fairbank NJ, Fabry B, Smith PG, Maksym GN. Localized mechanical stress induces time-dependent actin cytoskeletal remodeling and stiffening in cultured airway smooth muscle cells. Am J Physiol Cell Physiol 2004;287:C440-8.

26. Fulginiti J,3rd, Singer HA, Moreland RS. Phorbol ester-induced contractions of swine carotid artery are supported by slowly cycling crossbridges which are not dependent on calcium or myosin light chain phosphorylation. J Vasc Res 1993;30:315-322. 
27. Rhee AY, Brozovich FV. Force maintenance in smooth muscle: analysis using sinusoidal perturbations. Arch Biochem Biophys 2003;410:25-38.

28. Kuo KH, Wang L, Pare PD, Ford LE, Seow CY. Myosin thick filament lability induced by mechanical strain in airway smooth muscle. J Appl Physiol 2001;90:18111816.

29. Gunst SJ, Tang DD, Opazo Saez A. Cytoskeletal remodeling of the airway smooth muscle cell: a mechanism for adaptation to mechanical forces in the lung. Respir Physiol Neurobiol 2003;137:151-168.

30) Smolensky AV, Ragozzino J, Gilbert SH, Seow CY, Ford LE. Length-dependent filament formation assessed from birefringence increases during activation of porcine tracheal muscle. J Physiol 2005;563:517-527.

31. Qi D, Mitchell RW, Burdyga T, Ford LE, Kuo KH, Seow CY. Myosin light chain phosphorylation facilitates in vivo myosin filament reassembly after mechanical perturbation. Am J Physiol Cell Physiol 2002;282:C1298-305.

32. Sakai H, Chiba Y, Hirano T, Misawa M. Possible involvement of CPI-17 in augmented bronchial smooth muscle contraction in antigen-induced airway hyperresponsive rats. Mol Pharmacol 2005;68:145-151.

33. Sakai H, Hirano T, Chiba Y, Misawa M. Acetylcholine-induced phosphorylation and membrane translocation of CPI-17 in bronchial smooth muscle of rats. Am J Physiol Lung Cell Mol Physiol 2005;289:L925-30.

34. Houle F, Poirier A, Dumaresq J, Huot J. DAP kinase mediates the phosphorylation of tropomyosin-1 downstream of the ERK pathway, which regulates the formation of stress fibers in response to oxidative stress. J Cell Sci 2007;120:3666-3677.

35. Greenberg MJ, Wang CL, Lehman W, Moore JR. Modulation of actin mechanics by caldesmon and tropomyosin. Cell Motil Cytoskeleton 2008;65:156-164.

36. Janssen RA, Veenstra KG, Jonasch P, Jonasch E, Mier JW. Ras- and Raf-induced down-modulation of non-muscle tropomyosin are MEK-independent. J Biol Chem 1998;273:32182-32186.

37. Mitchell RW, Dowell ML, Solway J, Lakser OJ. Force fluctuation-induced relengthening of acetylcholine-contracted airway smooth muscle. Proc Am Thorac Soc 2008;5:68-72.

38. An SS, Fabry B, Mellema M, Bursac P, Gerthoffer WT, Kayyali US, Gaestel M, Shore SA, Fredberg JJ. Role of heat shock protein 27 in cytoskeletal remodeling of the airway smooth muscle cell. J Appl Physiol 2004;96:1701-1713. 
39. Lavoie TL, Dowell ML, Lakser OJ, Gerthoffer WT, Fredberg JJ, Seow CY, Mitchell $\mathrm{RW}$, Solway J. Disrupting actin-myosin-actin connectivity in airway smooth muscle as a treatment for asthma? Proc Am Thorac Soc. 2009:6(3):295-300. 


\section{CHAPTER 4. HUMAN AIRWAY SMOOTH MUSCLE IS STRUCTURALLY AND MECHANICALLY SIMILAR TO THAT OF OTHER SPECIES ${ }^{1}$}

\subsection{Introduction}

While the physiological function of airway smooth muscle (ASM) is unclear [1-3], its contraction and subsequent shortening act to increase airway resistance in the lung. Asthma is characterized by episodes of increased airway resistance due to exaggerated airway narrowing. Although it is generally agreed that the narrowing is caused by ASM shortening, it is still unclear if the excessive narrowing is due to fundamental changes in the phenotype of the smooth muscle itself, or is caused by structural and/or mechanical changes in the non-contractile elements of the airway wall, or by alterations in the relationship of the airway wall to the surrounding lung parenchyma [4]. One major hurdle in determining whether ASM is truly dysfunctional in asthma is the lack of adequate data on the mechanical properties of human ASM. Much of our current knowledge about ASM contractile function has come from studies in other mammalian species, which may not adequately represent human tissue. Hence, in order to investigate a potential role for ASM in asthma it is first necessary to characterize the mechanical properties of nonasthmatic ASM. Since many hypotheses concerning the pathogenesis of asthma have been developed from animal models, it is necessary to determine the relevance of these animal models with respect to human tissues. Thus, the goal of this study was to describe the mechanical properties and ultrastructure of non-asthmatic ASM and compare the data

\footnotetext{
${ }^{1}$ A version of this chapter has been accepted for publication. Chin LY, Bossé Y, Jiao Y, Solomon D, Hackett TL, Paré PD, Seow CY. (2009) Human airway smooth muscle is structurally and mechanically similar to that of other species. Eur Respir J. In Press.
} 
to previous human and mammalian studies of this tissue.

In the relatively few descriptions of non-asthmatic human ASM properties, a consistent finding has been higher passive tension, lower active force (and stress), and less shortening compared with ASM preparations from other animal species [5, 6]. However the results of these studies may be limited due to the fact that the tissues were obtained mostly from bronchial surgical resections [5-12, 32-41]. In the current study the ASM was carefully dissected from the tracheas of intact lungs donated for research.

Besides measuring the "classical" force-length and force-velocity relationships, we have focused on a newly recognized property of ASM, namely its plastic adaptability to the dynamic lung environment. None of the previous studies on mechanical properties of human ASM have studied plastic adaptation of the tissue. Importantly, in this study we used the in situ muscle length as the reference length for the muscle instead of the previous convention of $\mathrm{L}_{\max }$ (the length at which force was maximal at a given instant) [13].

\subsection{Materials and Methods}

\subsubsection{Tissue Preparation and Equilibration}

Tracheas were removed from non-transplantable human lungs donated for research through the International Institute for the Advancement of Medicine (IIAM: Edison, NJ). All donors had no known respiratory disease and their deaths were sudden. The subject demographics and clinical details are shown in Table 4.1. The study was approved by the 
UBC-St. Paul's Hospital Ethics Committee (Appendix A). The whole lungs were obtained as previously described [14]. Briefly, after surgical removal the lungs were flushed with Custodiol HTK solution (Odyssey Pharmaceuticals: East Hanover, NJ) and transported by plane on ice. The average time between harvesting and arrival at the University of British Columbia was 15-20 h.

The procedure of dissection and preparation of the smooth muscle was the standard procedure for sheep tissue and has been previously described [for details see 15, 16]. Briefly, the tracheal tissue was kept at $4^{\circ} \mathrm{C}$ in physiological saline solution (PSS). Dissection was undertaken within a day of obtaining the tissue. The in situ length of the tracheal smooth muscle was used as a reference length $\left(\mathrm{L}_{\mathrm{ref}}\right)$, which was determined prior to cutting open the $\mathrm{C}$-shaped cartilage ring. Connective tissue and epithelium was carefully removed to isolate a smooth muscle bundle. Muscle strips measuring 1-1.5mm wide, $0.5 \mathrm{~mm}$ thick, and $6 \mathrm{~mm}$ long, were attached on both ends with aluminum foil clips and mounted vertically on a force-length transducer. Before beginning the experimental protocol, the muscle was equilibrated at $\mathrm{L}_{\text {ref }}$ by periodic electrical field stimulation (EFS) at 5 -minute intervals for a period of $1.5 \mathrm{~h}$. Since some tissues exhibited substantial leukotriene-mediated tone without extrinsic stimulation, the CysLT1 receptor antagonist montelukast $\left(10^{-6} \mathrm{M}\right)$ was added to the PSS for all of the experiments, which prevented or eliminated tone. 
Table 4.1. Subject demographics and clinical details.

\begin{tabular}{|c|c|c|c|c|c|c|c|c|}
\hline Sex & Age & $\begin{array}{c}\text { Weight } \\
\text { (kg) }\end{array}$ & $\begin{array}{l}\text { Height } \\
\text { (cm) }\end{array}$ & Ethnicity & Cause of Death & $\begin{array}{l}\text { Patient Medical } \\
\text { History }\end{array}$ & Known Meds & Terminal Meds \\
\hline $\mathrm{M}$ & 4 & 17.5 & 104 & Hispanic & Head trauma & None & None & $\begin{array}{l}\text { Steroids and } \\
\text { vasopressors }\end{array}$ \\
\hline $\mathrm{M}$ & 22 & 112 & 193 & Caucasian & Head trauma & $\begin{array}{l}\text { Occasional marijuana } \\
\text { (once a year) }\end{array}$ & None & Vasopressors \\
\hline $\mathrm{F}$ & 63 & 77 & 159 & Caucasian & $\begin{array}{c}\text { Gastrointestinal } \\
\text { bleeding }\end{array}$ & $\begin{array}{c}\text { Hypertension X } 2 \\
\text { years }\end{array}$ & $\begin{array}{c}\text { Unknown } \\
\text { antihypertensive }\end{array}$ & Dopamine \\
\hline$F$ & 19 & 87 & 165 & Caucasian & Head trauma & None & None & $\begin{array}{l}\text { Steroids and } \\
\text { vasopressors }\end{array}$ \\
\hline $\bar{F}$ & 20 & 72.2 & 165 & Caucasian & Head trauma & $\begin{array}{c}\text { Smoked cigarettes }< \\
1 \text { PPD } \dagger \text { for } 2 \text { years. } \\
\text { Marijuana smoked } \\
\text { unknown frequency. }\end{array}$ & $\begin{array}{l}\text { Pain medications } \\
\text { and inhalants }\end{array}$ & Vasopressors \\
\hline $\mathrm{M}$ & 24 & 81.8 & 175 & Hispanic & Head trauma & None & None & Vasopressors \\
\hline
\end{tabular}

$\dagger$ Pack per day 


\subsubsection{Mechanical Measurements}

After equilibration, the maximal isometric force produced in response to EFS at $\mathrm{L}_{\text {ref }}$ was determined (herein called $\mathrm{F}_{\max }$ ). For the force-length relationship the muscle was either stretched or shortened in a step-wise fashion as previously described $[15,17]$ and allowed to adapt to every new length for 20 minutes during which it was stimulated with EFS at 5minute intervals. Five different lengths were examined: $0.5,0.75,1.0,1.25$, and $1.5 \mathrm{~L}_{\text {ref. }}$. To determine the response to length perturbation, a 10 minute, $0.2 \mathrm{~Hz}, 30 \% \mathrm{~L}_{\text {ref }}$ length oscillation was applied. The recovery of EFS-induced (active) force was followed for 30 minutes after oscillation by stimulating with EFS at 5-minute intervals. For the forcevelocity curves, velocity measurements were made after release (quick switch from isometric to isotonic contraction) at five graded loads (between zero and $\mathrm{F}_{\max }$ ) at $\mathrm{L}_{\mathrm{ref}}$ as previously described [18]. Velocities were measured at two time points: one at the peak of tetanic force and one mid-way to the peak. This is due to the non-linear nature of shortening velocity, which peaks early on as developed force reaches $\sim 50 \%$ of $\mathrm{F}_{\max }$, and settles to a lower plateau after the force reaches $F_{\max }$ [19]. Shortening velocity against a given load was recorded $100 \mathrm{msec}$ after the release, during the steady-phase of shortening. The curves were fit using Hill's hyperbolic equation [20]. Maximal isotonic shortening was established by allowing a muscle to contract against a preload equal to $10 \%$ and $20 \%$

$\mathrm{F}_{\max }$. It was not possible to perform the shortening at zero-load because the servo-system of the myograph became unstable at low loads. Therefore, the maximal shortening was extrapolated to zero load from these two points. 


\subsubsection{Histology and Electron Microscopy}

At the end of the mechanical protocols the tissue preparations were fixed at $\mathrm{L}_{\text {ref }}$ in $10 \%$ formalin for histology or a formaldehyde cocktail for transmission electron microscopy (EM). The histology was performed as previously described [21]. The amount of muscle in the preparation was determined by staining transverse sections with Masson's trichrome and quantified by manual tracing using Image ProPlus 4.5 (MediaCybernetics: Bethesda, MD). Maximal stress generating capacity was determined by dividing $\mathrm{F}_{\max }$ (in $\mathrm{mN}$ ) by the cross-sectional area (in $\mathrm{mm}^{2}$ ) of muscle present in the preparation. The protocol for EM followed the standard procedure previously described in our lab (see [23] for details).

\subsubsection{Statistical Analysis}

Force and length measurements were normalized to $\mathrm{F}_{\max }$ or $\mathrm{L}_{\text {ref }}$ respectively and expressed as fractions of $\mathrm{F}_{\max }$ and $\mathrm{L}_{\text {ref. }}$ Velocity of shortening was expressed as $\mathrm{L}_{\text {ref }} / \mathrm{sec}$. Aggregate data were expressed as mean \pm SEM. ANOVA and regression analyses were accomplished using GraphPad Prism 5 (GraphPad Software, Inc.: La Jolla, CA). p $\leq 0.05$ was considered to be sufficient to reject the null hypothesis.

\subsection{Results}

\subsubsection{Muscle Bundle Properties and Morphology}

Human lungs were received from six donors with an average age of $25.8 \pm 2.8$ years. After measurements of mechanical properties, muscle strips were fixed and stained with Masson's Trichrome for histological morphometry $(n=4)$. From the histological sections 
the cross-sectional area of the muscle was measured and compared to the entire tissue preparation (Fig. 4.1). The percentage of smooth muscle cross-sectional area to the total cross-sectional area of the tissue preparation averaged $36.5 \pm 0.04 \%$. The maximal stress generated by the muscle averaged $82.1 \pm 17.3 \mathrm{mN} / \mathrm{mm}^{2}$.

\subsubsection{Ultrastructure}

Muscle strips were also fixed for EM. A transverse electron micrograph of a muscle cell is shown in Figure 4.2. The cells are similarly 'packed' within bundles like ASM from other species (Fig. 4.2), and share similar intracellular features. Like other mammalian ASM cells, human cells lack the well-organized arrays of contractile filaments seen in striated muscle (Fig. 4.2 and 4.3). Myosin thick filaments are present throughout the cell and are vastly outnumbered by actin filaments (Fig. 4.2 and 4.3). Areas of electron dense material, known as dense bodies and plaques, are also seen. Mitochondria, sarcoplasmic reticula, caveolae, and microtubules are also present and are indistinguishable from their counterparts in animal cells. 


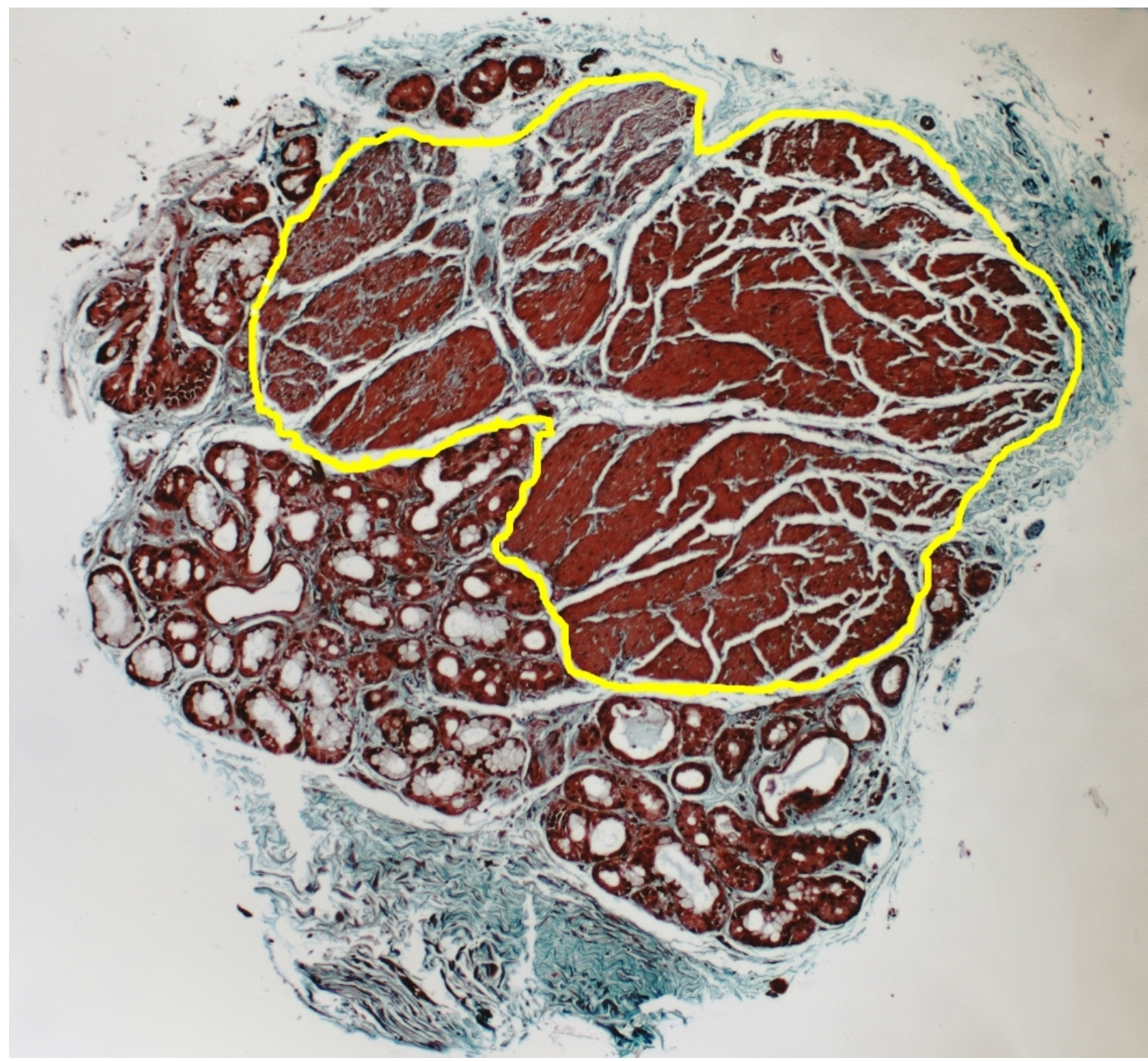

Figure 4.1 Histological section of airway smooth muscle. ASM strips were stained with

Masson's Trichrome. The smooth muscle is red, connective tissue blue, and nuclei black. The yellow outline encircles the muscle bundles. The glandular, red staining cells were excluded. Morphometric analysis was used to determine the cross-sectional area of muscle in the dissected strip. This area was used to calculate the maximal stress generated by each smooth muscle preparation $($ stress $=$ force/cross sectional area). Color segmentation was used to exclude the blue connective tissue within muscle bundles. Image width $=1.14$ $\mathrm{mm}$. 


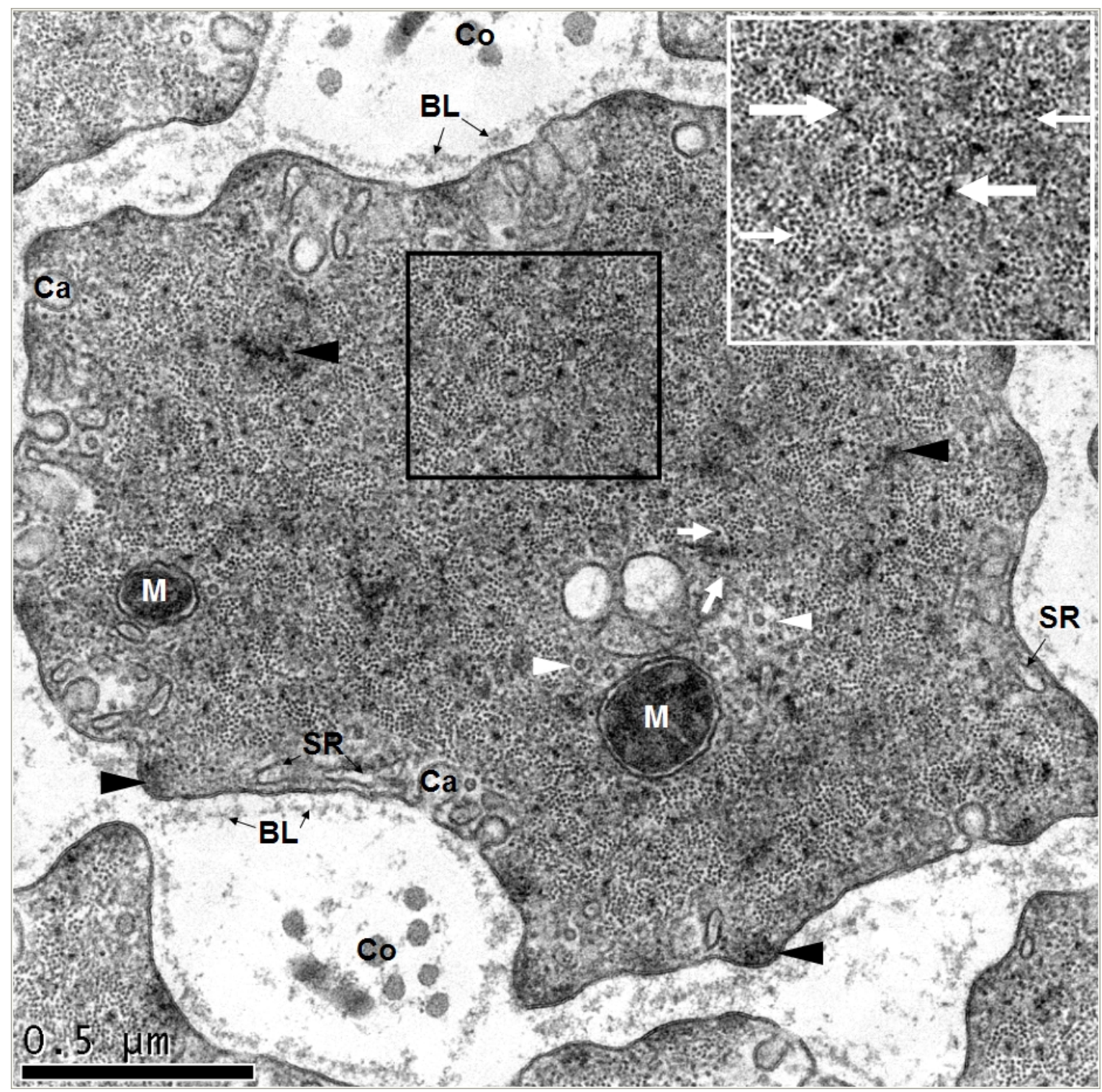

Figure 4.2 Transverse electron micrograph of an ASM cell. Black arrowheads indicate dense bodies (cytoplasmic) and dense plaques (membrane associated). White arrows indicate intermediate filaments; white arrowheads indicate microtubules. M: Mitochondria; Ca: Caveolae; SR: Sarcoplasmic reticulum; BL: Basal lamina; Co: Collagen fibers. Inset: Magnification of the black square. Small arrows indicate actin thin filaments and larger arrows indicate myosin thick filaments. Bar $=0.5 \mu \mathrm{m}$. 


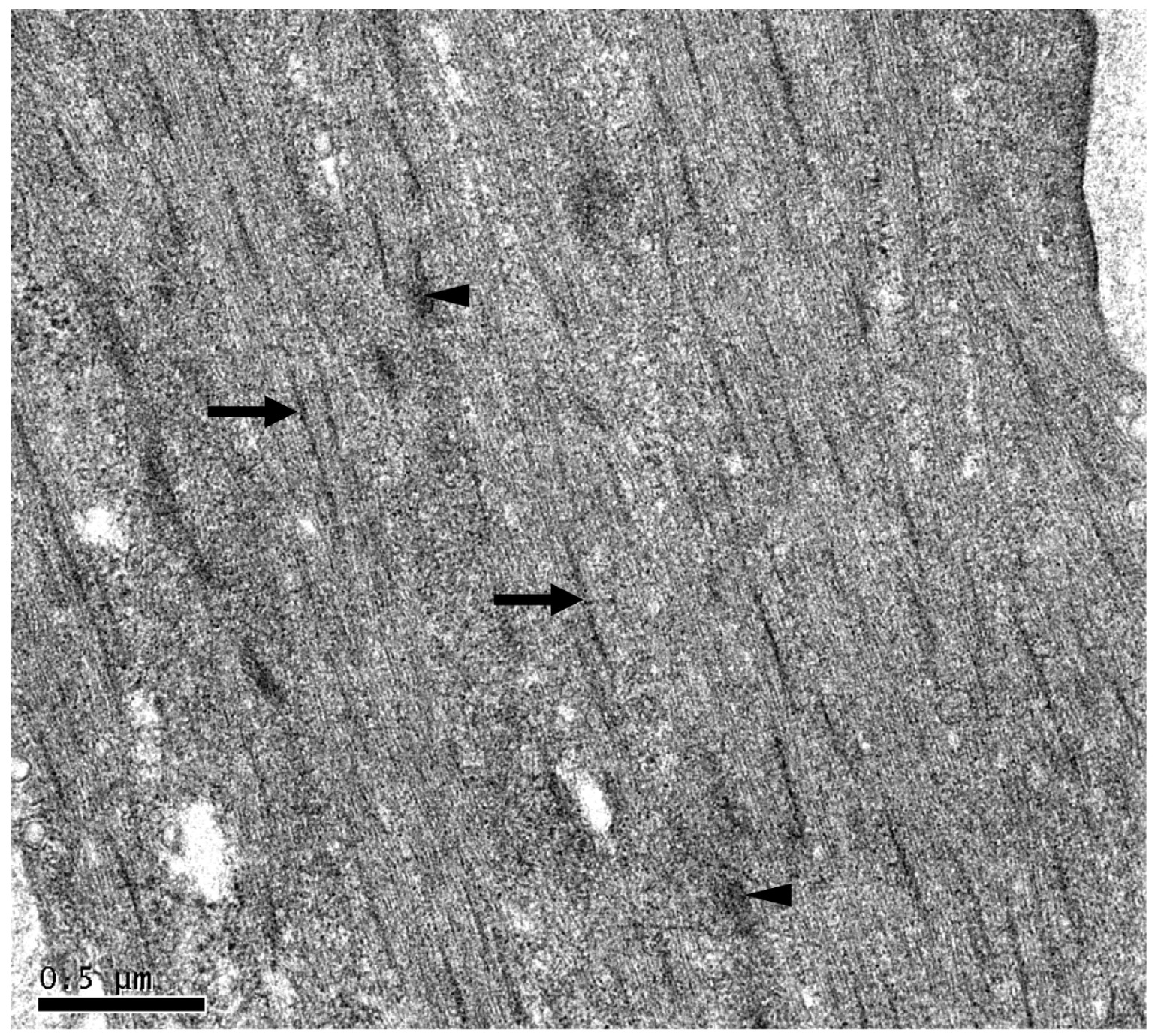

Figure 4.3 Electron micrograph of a longitudinal ASM cell section. Arrowheads point to dense bodies and arrows point to myosin filaments. Bar $=0.5 \mu \mathrm{m}$. 


\subsubsection{Force-Length Properties and Length Adaptation}

The relationship between muscle length and force generating capacity was examined by recording force at five different lengths (Fig. 4.4). Between shorter and longer lengths the muscle was returned to $\mathrm{L}_{\text {ref }}$ and re-equilibrated (Fig. 4.4A). Immediately following a length change from $\mathrm{L}_{\mathrm{ref}}$, the active force declined and gradually recovered to a greater force over time (Fig. 4.4B). On average, the active force did not fully recover to $F_{\max }$ at any of the lengths during the 20 minutes. Part of the incomplete recovery was due to a general force deterioration over the time course of the experiment. Correction for force deterioration was accomplished by assuming a linear decline and fitting a line through the first contraction at $\mathrm{L}_{\text {ref }}$ and the last (fifth) contraction after the length was returned to $\mathrm{L}_{\text {ref }}$ (force $=-0.0006 \mathrm{x}$ time +1 , where time is in minutes; suggesting an average decline of $10.8 \%$ from initial force after $3 \mathrm{~h}$ ). This correction assumes that force at $\mathrm{L}_{\text {ref }}$ returns to $\mathrm{F}_{\max }$ in the absence of deterioration, thus preventing an underestimation of force recovery (corrected values are presented in Figs. 4.4 and 4.5). To compare the extent of length adaptation, the initial force after a length change (first contraction) was compared to the final force measurement after the adaptation period (fifth contraction), this is displayed in Figure 4.5. There was a significant length adaptation of active force over the 20 minute period (Fig. 4.5) (ANOVA: $\mathrm{p}<0.0001$ ). Bonferroni posttests demonstrated that active force at $0.50 \times \mathrm{xL}_{\text {ref }}$ and $1.50 \times \mathrm{xL}_{\text {ref }}$ were significantly greater after the period of adaptation $(\mathrm{p}<0.05$ and $\mathrm{p}<0.001$, respectively). Passive force (Fig. $4.4 \mathrm{C}$ and 4.5 ) also demonstrated a significant length adaptation (ANOVA: $\mathrm{p}=0.0053$ ). At lengths longer than $\mathrm{L}_{\mathrm{ref}}$, the passive

force initially increased with the length change but decreased over the period of adaptation. Conversely, at shorter lengths, the passive force initially declined but 
gradually increased over the 20 minute period. On average the passive force did not reach pre-length change levels despite significant adaptation (Fig. 4.5). 
Figure 4.4 Isometric force at different lengths. A) The sequence and the time course of length changes are shown. ASM strips were either shortened or lengthened in the relaxed state to $0.50,0.75,1.25$ or $1.50 \mathrm{~L}_{\text {ref }}$. The muscle was stimulated to contract 5 times with EFS at 5-minute intervals after every length change. The force was corrected for force deterioration over time (see text for details). Between shortening and lengthening steps, the muscle was returned to, and readapted at, $\mathrm{L}_{\text {ref. }}$ Black hexagons indicate the time points where EFS occurred. The active force (B) and passive force (C) were recorded at every contraction. Error bars indicate SEM, $\mathrm{n}=6$ from 6 different donors. 


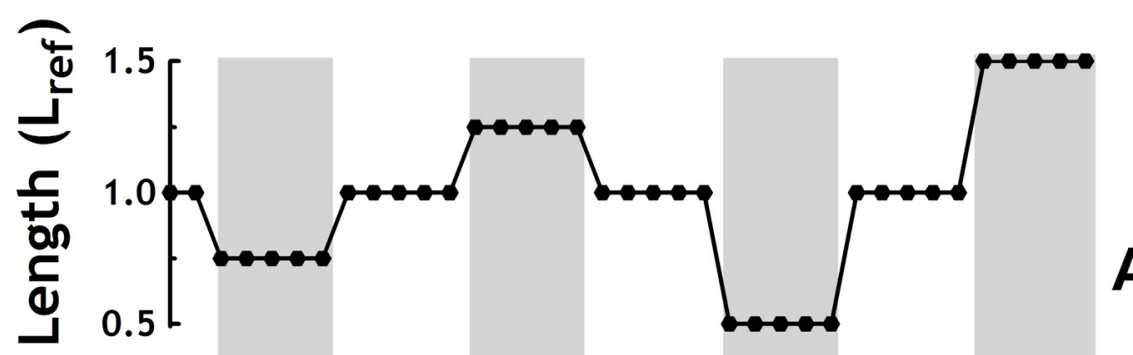

A

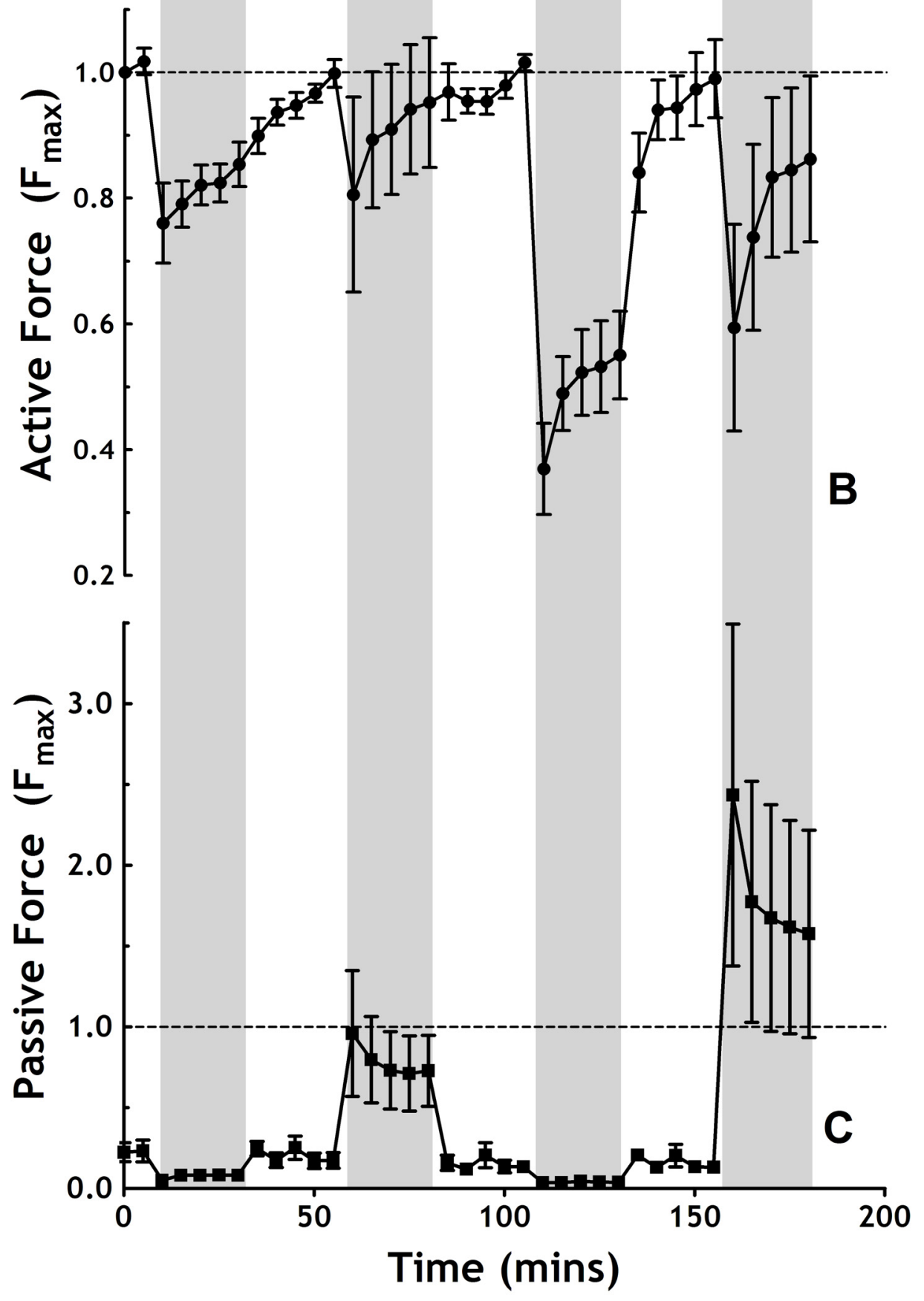




\subsubsection{Recovery from Mechanical Perturbation}

The magnitude and time required for force recovery following a perturbation was examined by applying an oscillatory strain to the relaxed muscle. The ten minute length oscillation occurred at a frequency that mimics human breathing $(0.2 \mathrm{~Hz})$ and at a magnitude similar to deep inspiration $\left(30 \% \mathrm{~L}_{\mathrm{ref}}\right)$. As seen in Figure 4.6 (not corrected for force deterioration), immediately following oscillation the force decreased to $0.643 \pm 0.03$ $\mathrm{F}_{\max }$ and recovered to $0.953 \pm 0.03 \mathrm{~F}_{\max } 30$ minutes after oscillation.

\subsubsection{Shortening Velocity}

Two sets of data were generated to examine the shortening velocity of human ASM. At both time points, the muscle was released to five different pre-determined loads (each representing a certain percentage of $\left.F_{\max }\right)$. The difference between the two sets of data was the time of release (Fig. 4.7). The late releases were performed during the tetanic plateau of active force while the early releases were performed mid-way to the plateau (on average between 3 to 4 seconds after the onset of stimulation). Both data sets were fitted with Hill's hyperbolic equation [20] (Fig. 4.8). Maximal shortening velocity ( $\left.\mathrm{V}_{\max }\right)$ determined by Hill's equation was 0.609 and $0.394 \mathrm{~L}_{\mathrm{ref}} / \mathrm{s}$ for the early and late-phase, respectively. Force-velocity curves at the two time points were significantly different (repeated measures ANOVA: $\mathrm{p}<0.0001$ ). 


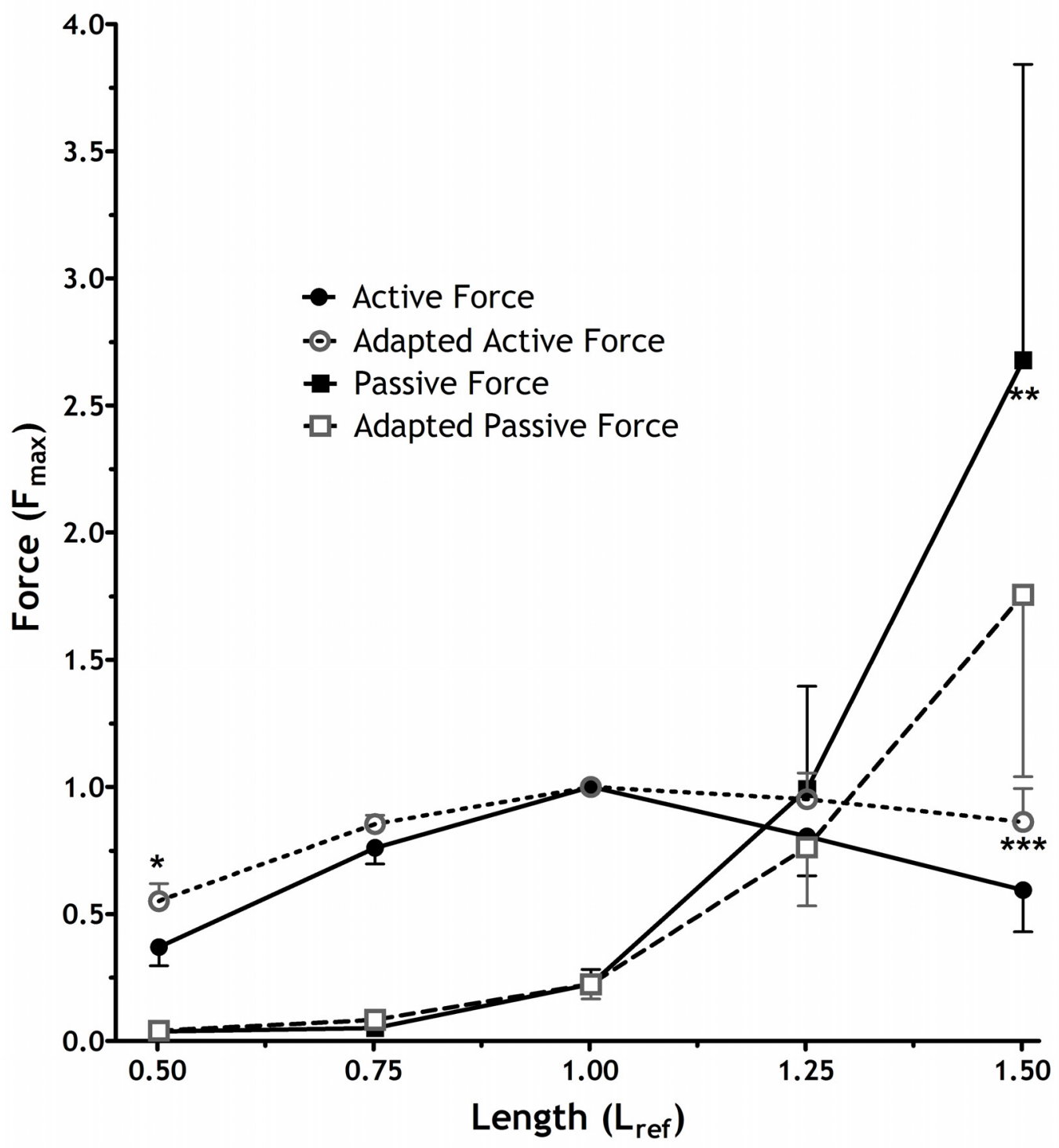

Figure 4.5 Active and passive force-length relationships before and after length adaptation. From $\mathrm{L}_{\text {ref, }}$ the ASM strips were either shortened or lengthened to $0.50,0.75$, 1.25, and $1.50 \mathrm{~L}_{\text {ref }}$ (refer to Fig. 4.4 for sequence and time course of length changes and stimulation). Active force (circles) and passive force (squares) both before (solid lines and closed symbols) and after (dashed lines and open symbols) length adaptation are shown. The force was corrected for deterioration over the time period of the experiment, 
see text for details. Active force before and after adaptation was length independent in the range $0.75-1.5 \mathrm{~L}_{\text {ref }}($ ANOVA, $\mathrm{p}<0.05)$. Error bars indicate $\mathrm{SEM}, \mathrm{n}=6$ from 6 different donors. $*: \mathrm{p}<0.05, * *: \mathrm{p}<0.01,{ }^{* * *}: \mathrm{p}<0.001$; adapted force compared to nonadapted force at a given length.

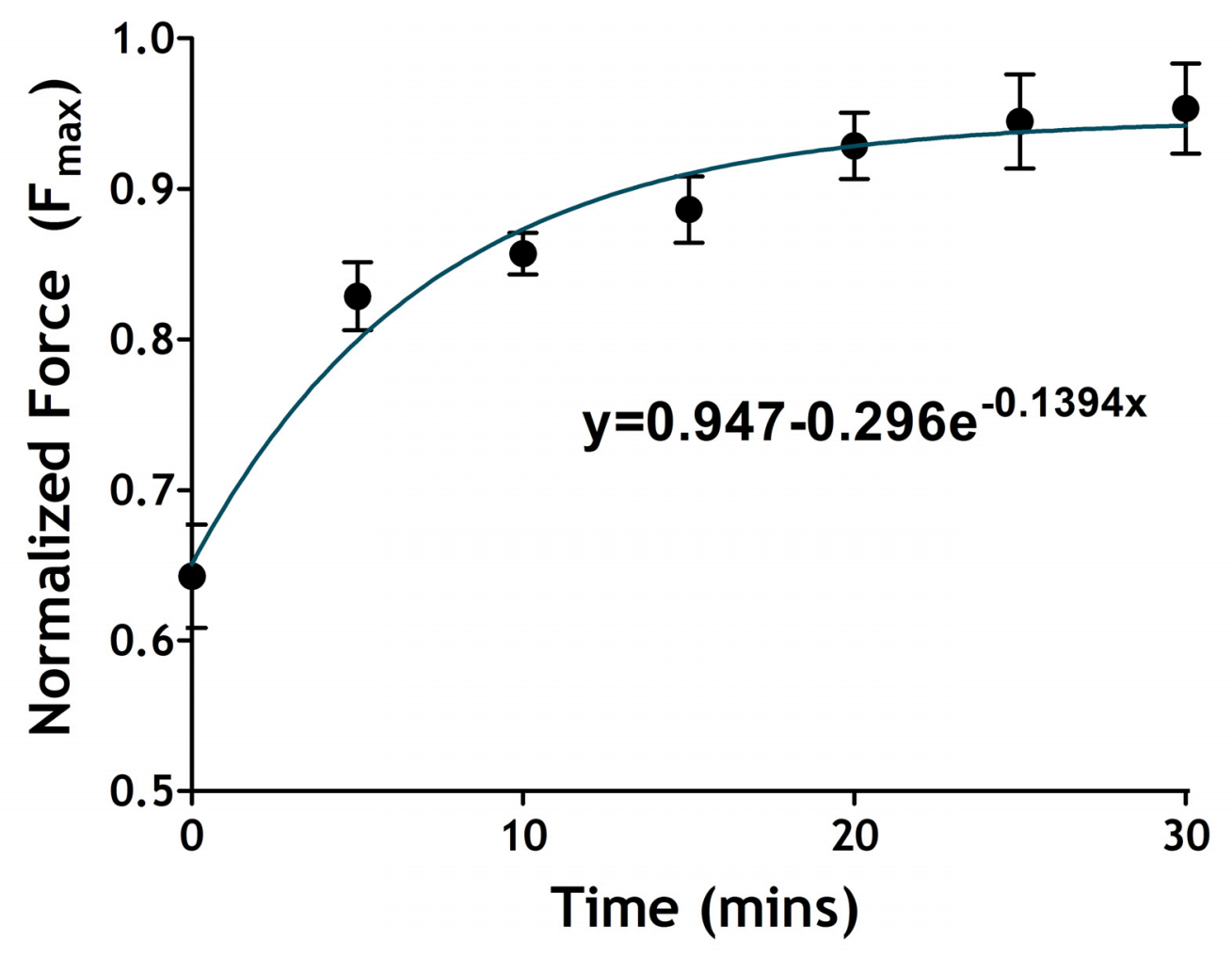

Figure 4.6 Isometric force recovery following a length oscillation. Recovery of isometric force following a ten-minute, $0.2 \mathrm{~Hz}, 30 \% \mathrm{~L}_{\text {ref }}$ length oscillation. ASM strips were adapted to $\mathrm{L}_{\text {ref }}$ prior the oscillation. The force produced by seven EFS-induced contractions were recorded at 5-minute intervals following the oscillation. Error bars indicate SEM, $\mathrm{n}=6, \mathrm{R}^{2}=0.7254$. 

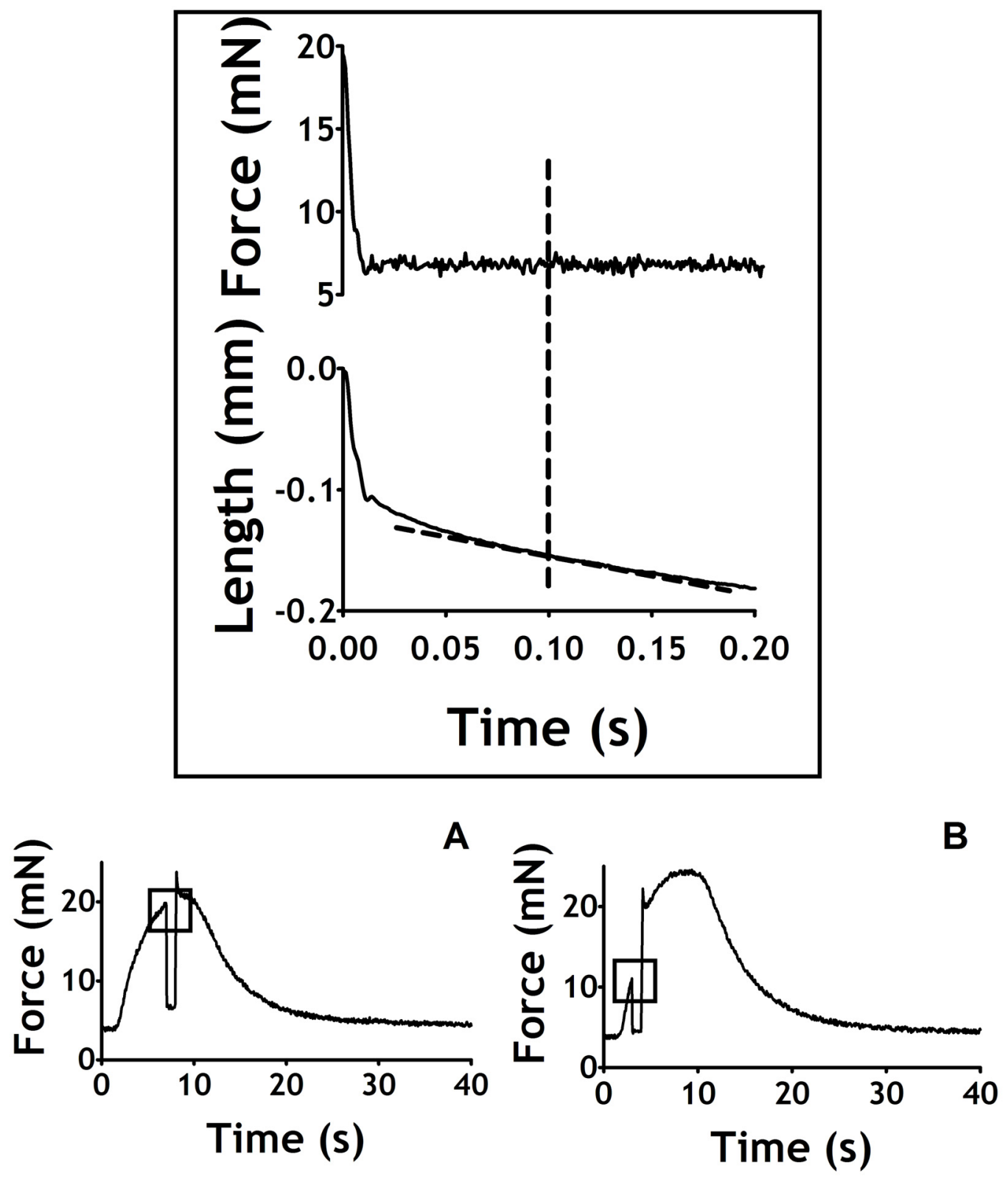

Figure 4.7 Determining shortening velocity during an isotonic contraction. Shortening velocity was determined at two time points during EFS (A and B). A late load release was performed during the force plateau (A), while an early load release was performed midway to the force plateau (B). The top panel shows representative traces of force and length records of an isotonic release. The load against which the muscle 
contracted was reduced to a specific load in a time space of $10 \mathrm{~ms}(6 \mathrm{mN}$ in the case shown here). The velocity was determined by calculating the slope of the tangent line during steady-state shortening at $100 \mathrm{~ms}$. For each release, five different loads were used.

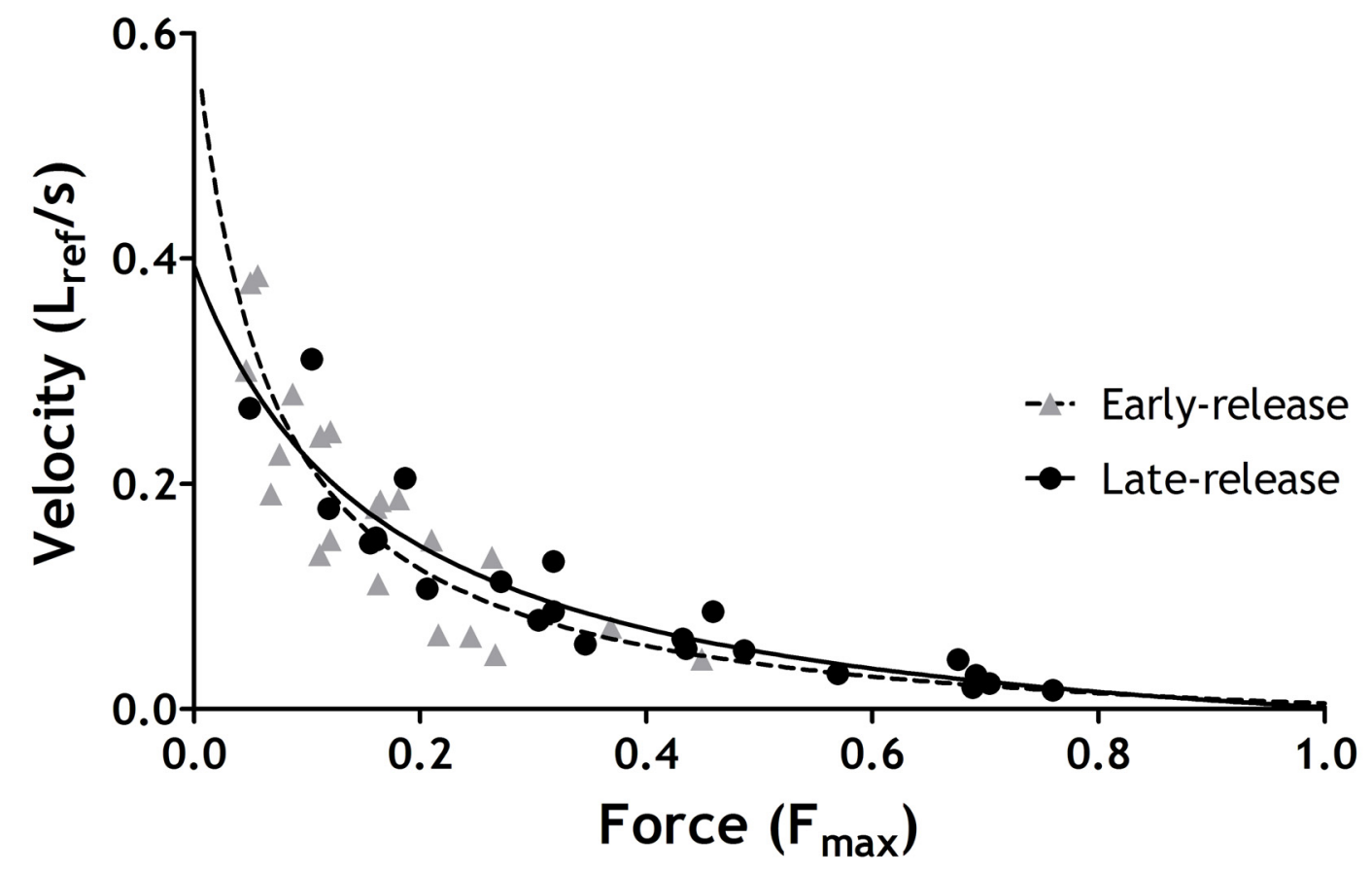

Figure 4.8 Isotonic shortening velocity measured against different loads at two time points. Isotonic shortening velocity was measured at five different loads both at the early and the late phase of contraction (refer to Fig. 4.7 for an example of shortening velocity measurement). The loads were determined as a percentage of $F_{\max }$. Late release (black circles) and early release (grey triangles) data were fitted with Hill's hyperbolic equation. $\mathrm{n}=5$ from 5 different donors. Late-phase $\mathrm{R}^{2}=0.8665$, early-phase $\mathrm{R}^{2}=0.8417$. The two groups were significantly different, repeated measures ANOVA: $p<0.0001$. 


\subsubsection{Maximal Isotonic Shortening}

In four muscle preparations the extent of unloaded shortening was determined (Fig. 4.9). Each ASM strip was allowed to isotonically shorten against a predetermined load $(10 \%$ and $\left.20 \% \mathrm{~F}_{\max }\right)$. The maximal unloaded shortening was extrapolated from a linear regression of the points $\left(\mathrm{R}^{2}=0.749, \mathrm{p}=0.0055\right)$. This regression assumes that the ascending limb of the force-length curve for a non-adapted muscle is linear [25]. The 95\% confidence intervals ranged from 60.3 to $84.1 \%$ of shortening at zero-load with an average of $72.2 \%$ (total length of the muscle).

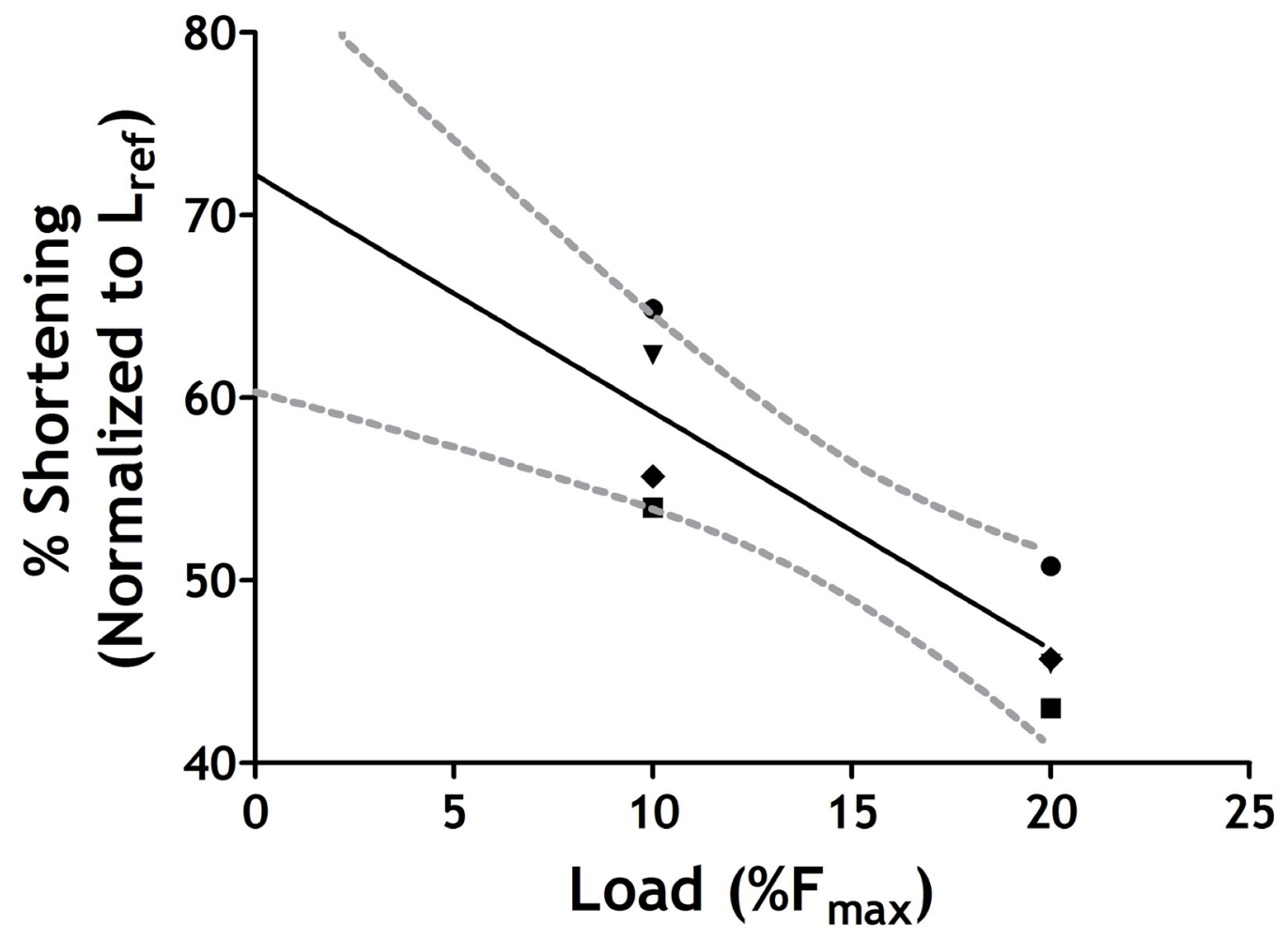

Figure 4.9 Maximal shortening. ASM strips were stimulated to contract with EFS and the total amount of isotonic shortening against low loads was measured. Using the 
amount of shortening recorded at loads representing $10 \%$ and $20 \%$ of $\mathrm{F}_{\max }$, maximal shortening was extrapolated. Each symbol represents a muscle from a unique donor, $n=4$ from 4 different donors. $\mathrm{R}^{2}=0.7488, \mathrm{p}=0.0055$.

\subsection{Discussion}

Despite the prominent role of ASM in airway diseases, the basic mechanical properties of human ASM have not been adequately determined. This study is the most comprehensive description of the mechanical properties of human ASM to-date and is unique in that the experiments were performed within the paradigm of ASM mechanical plasticity using a high quality tissue source. Our results demonstrate that human ASM possesses similar mechanical properties and morphological features as those found in other mammals such as dogs, pigs, and sheep. This provides justification for using ASM tissues from nonhuman species to elucidate contractile mechanisms. Also, mechanical responses to experimental interventions in non-human mammalian ASM can now be justifiably interpreted in terms of human airway physiology. This study also provides a database of mechanical parameters for intact non-asthmatic human ASM, an invaluable reference against which asthmatic ASM can be compared.

Qualitatively, human ASM is indistinguishable from that of other species, as concluded from comparisons with ultrastructural images $[15,22,25,29]$. The present study demonstrates the feasibility for quantitative comparisons of ultrastructural features (e.g. filament densities) and the relative abundance of mitochondria, caveolae, sarcoplasmic reticula, and other organelles of interest. Comparisons of these features will provide 
invaluable insights into the phenotypic changes associated with asthmatic ASM.

The results of previous studies suggested that human ASM possesses reduced stress generating capacity, reduced shortening capacity, and greater passive stiffness compared to other species $[5,6]$. Human ASM was found to generate a maximal stress of $50 \pm 20$ $\mathrm{mN} / \mathrm{mm}^{2}$ compared to 140,72 , and $80 \mathrm{mN} / \mathrm{mm}^{2}$ in rabbit, dog, and swine ASM, respectively [6]. The present results show that human ASM $\left(82 \pm 17.3 \mathrm{mN} / \mathrm{mm}^{2}\right)$ generates stress which is comparable to dog and swine ASM. Another major finding of the present study is the ability of human ASM to undergo extensive shortening (72\%) (Fig. 4.9). This degree of shortening is similar to the reported values in other animal species which range from 61 to $71 \%$, and is much greater than the previously reported human ASM value of $25 \pm 9.0 \%$ [6]. This discrepancy could be related to the reduced stress generated by the tissues in earlier studies which were dissected from bronchi obtained from surgical resections. In addition, the starting length of the muscle was set at $\mathrm{L}_{\max }$, an arbitrary length that is greatly influenced by the state of adaptation of the muscle [13].

Another difference in the present study, which could influence the degree of maximal shortening, was the percentage of muscle to total tissue area $(36.49 \pm 0.04 \%)$ which is much greater than the $8.7 \%$ reported in preparations from earlier human studies [5]. These data suggested that the previously studied human ASM was surrounded by a greater proportion of connective tissue resulting in higher passive stiffness, when compared with other species. Increased connective tissue could also prevent shortening by acting as a radial constraint and/or compressive load in parallel to the muscle cells [28]. This 
hypothesis is supported by the observation that treating human ASM preparations with collagenase, led to a $50 \%$ increase in maximal isotonic shortening $[11,12]$. Our carefully dissected tracheal preparations had similar ASM content to other non-human preparations which ranged from $25-35 \%$ smooth muscle area [6]. While the decreased connective tissue surrounding the muscle may have increased shortening in our study, it is unclear if passive tension was affected. In the previous study conducted at $\mathrm{L}_{\max }$, the passive tension in the muscle preparation was $60 \pm 8.8 \%$ of maximal force [5]. In the present study average passive tension at $\mathrm{L}_{\text {ref }}$ was $22.4 \pm 0.1 \% \mathrm{~F}_{\max }$.

Shortening velocity in smooth muscle peaks early in contraction before force reaches a plateau; the velocity then decreases to a lower level after force plateaus and this velocity is maintained during the sustained phase of contraction. This decrease in velocity has been attributed to the development of latch-bridges caused by dephosphorylation of the myosin regulatory light chain in arterial muscle [26]. In non-human ASM, it has been proposed that the decreased velocity is likely due to thick filament lengthening during force development [18] and myosin phosphorylation was not found to correlate to velocity [27]. The present finding that velocity declines (Fig. 4.8) during the time course of an isometric contraction suggests that myosin filaments in human ASM undergo similar rearrangements.

This is the first study to investigate whether length adaptation occurs in human ASM. Smooth muscle like striated muscle operates over a length range. In striated muscle the force-length relationship contains a well-defined optimal length where developed force is 
at its maximum. Beyond the narrow plateau of maximal force, the force developed by the muscle decreases at shorter and longer lengths. This relationship depends on the overlap of the actin and myosin filaments within the muscle. Likewise, smooth muscle displays a force-length relationship that approximately resembles a concave-down parabolic curve. However, airway smooth muscle producing 'suboptimal' force at a shorter or longer length can adapt to the new length, increasing its force within minutes [17]. This requires rearrangement of contractile and cytoskeletal proteins, and effectively broadens the forcelength relationship of the smooth muscle [24, 29]. Thus, length adaptation allows smooth muscle to operate over the large length ranges seen in vivo, particularly in the smooth muscle tissues that line hollow organs that undergo large volume changes. Compared to previous studies in canine ASM [17] our data suggests that human ASM is less adaptable, that is, the length range of the force plateau in human ASM is smaller than that of canine ASM. Nevertheless, maximal force in human ASM is length-independent over the range of $0.75-1.5 \mathrm{~L}_{\text {ref }}($ ANOVA, $\mathrm{p}<0.05)$, a clear sign that human ASM is capable of length adaptation (Fig. 4.5).

The ability of the muscle to undergo length adaptation suggests that human ASM is capable of the mechanical plasticity demonstrated in other mammalian species. This ability to rearrange intracellular elements to generate force is further supported by the muscle's response to length oscillation (Fig. 4.6). Our laboratory has previously shown in swine tissue that an oscillatory strain causes a decrease in force immediately after oscillation, followed by an exponential force recovery that coincides with myosin filament reformation [23]. However, the magnitude of recovery is somewhat blunted in human 
ASM since the force did not fully recover to $F_{\max }$, as it does in swine. Likewise, the rate of recovery is slower, with a rate constant of $0.139 \mathrm{~s}^{-1}$ versus $0.234 \mathrm{~s}^{-1}$ in swine ASM [23]. Human ASM is also more easily perturbed, since the force produced by human ASM immediately after oscillation was $64 \% \mathrm{~F}_{\max }$ compared to $\sim 79 \% \mathrm{~F}_{\max }$ in swine [23]. This implies that human ASM has a more labile intracellular structure that reorganizes slower than ASM from other species. In vivo, this may be beneficial for maintenance of airway patency. Coupled with its reduced ability to undergo length adaptation, the force loss associated with oscillation could enhance and prolong the effectiveness of bronchoprotection provided by a deep inspiration [30-31]. The increased sensitivity to mechanical perturbation could be related to differences in the muscle's contractile apparatus or due to differences in extracellular structures, perhaps exposing human ASM to greater internal forces than is seen by other species. Comparisons to ASM from asthmatic subjects are certainly needed.

Previous studies comparing asthmatic and non-asthmatic ASM mechanical properties have suggested increased contractility (force development or shortening) [12, 39, 41] or sensitivity to certain agonists in asthmatic ASM $[12,32,36]$, while others have shown no differences [33-35, 37, 38, 40]. With respect to the current study, it is unclear whether these are true differences or due to the inexactness of using $\mathrm{L}_{\max }$ to determine 'optimal force,' since the velocity, extent of shortening, and sensitivity to an agonist [42] are all length dependent. Only two studies have normalized force to cross-sectional muscle area, concluding that asthmatics generate more stress than non-asthmatics [12, 41]. Here we suggest that these studies may underestimate maximal stress and the extent of shortening, 
either because of the way $\mathrm{L}_{\max }$ was determined or tissue quality. These factors may have equally affected previous results in asthmatics and non-asthmatics and thus would not alter their conclusions. Alternatively, we could also speculate that our results may specifically affect non-asthmatic ASM measurements, since airway remodeling leading to increased connective tissue may decrease overall cell contractility despite the previous underestimation.

In conclusion, this study is the first comprehensive study of the mechanical properties of healthy human ASM sourced from an intact lung. We have demonstrated that human ASM has ultrastructural features and mechanical properties which are similar to other animal species and is capable of length adaptation. These results suggest that human ASM likely has the same intracellular organization and undergoes the same processes of mechanical plasticity that have been identified in the ASM of other species. 


\subsection{References}

1. Seow CY and Fredberg JJ. Historical perspective on airway smooth muscle: the saga of a frustrated cell. J.Appl.Physiol. 2001; 91: 2: 938-952.

2. Mead J. Point: airway smooth muscle is useful. J.Appl.Physiol. 2007; 102: 4: 1708-9; discussion 1710.

3. Fredberg JJ. Counterpoint: airway smooth muscle is not useful. J.Appl.Physiol. 2007; 102: 4: 1709-10; discussion 1710-1.

4. An SS, Bai TR, Bates JH, Black JL, Brown RH, Brusasco V, Chitano P, Deng L, Dowell M, Eidelman DH, Fabry B, Fairbank NJ, Ford LE, Fredberg JJ, Gerthoffer WT, Gilbert SH, Gosens R, Gunst SJ, Halayko AJ, Ingram RH, Irvin CG, James AL, Janssen LJ, King GG, Knight DA, Lauzon AM, Lakser OJ, Ludwig MS, Lutchen KR, Maksym GN, Martin JG, Mauad T, McParland BE, Mijailovich SM, Mitchell HW, Mitchell RW, Mitzner W, Murphy TM, Paré PD, Pellegrino R, Sanderson MJ, Schellenberg RR, Seow CY, Silveira PS, Smith PG, Solway J, Stephens NL, Sterk PJ, Stewart AG, Tang DD, Tepper RS, Tran T, Wang L. Airway smooth muscle dynamics: a common pathway of airway obstruction in asthma. Eur.Respir.J. 2007; 29: 5: 834-860.

5. Ishida K, Paré PD, Hards J, Schellenberg RR. Mechanical properties of human bronchial smooth muscle in vitro. J.Appl.Physiol. 1992; 73: 4: 1481-1485.

6. Opazo Saez AM, Schellenberg RR, Ludwig MS, Meiss RA, Paré PD. Tissue elastance influences airway smooth muscle shortening: comparison of mechanical properties among different species. Can.J.Physiol.Pharmacol. 2002; 80: 9: 865-871.

7. De Jongste J, Mons H, Van Strik R, Bonta I, Kerrebijn K. Human small airway smooth muscle responses in vitro; actions and interactions of methacholine, histamine and leukotriene C4. Eur.J.Pharmacol. 1986; 125: 1: 29-35.

8. De Jongste JC, Mons H, Block R, Bonta IL, Frederiksz AP, Kerrebijn KF. Increased in vitro histamine responses in human small airways smooth muscle from patients with chronic obstructive pulmonary disease. Am.Rev.Respir.Dis. 1987; 135: 3: 549-553.

9. de Jongste JC, van Strik R, Bonta IL, Kerrebijn KF. Measurement of human small airway smooth muscle function in vitro with the bronchiolar strip preparation. J.Pharmacol.Methods 1985; 14: 2: 111-118.

10. Jongejan RC, de Jongste JC, van Strik R, Raatgeep HR, Bonta IL, Kerrebijn KF. Measurement of human small airway smooth muscle function in vitro. Comparison of bronchiolar strips and segments. J.Pharmacol.Methods 1988; 20: 2: 135-142.

11. Bramley AM, Roberts CR, Schellenberg RR. Collagenase increases shortening of 
human bronchial smooth muscle in vitro. Am.J.Respir.Crit.Care Med. 1995; 152: $5 \mathrm{Pt}$ 1: $1513-1517$.

12. Bramley AM, Thomson RJ, Roberts CR, Schellenberg RR. Hypothesis: excessive bronchoconstriction in asthma is due to decreased airway elastance. Eur.Respir.J. 1994; $7: 2: 337-341$.

13. Bai TR, Bates JH, Brusasco V, Camoretti-Mercado B, Chitano P, Deng LH, Dowell M, Fabry B, Ford LE, Fredberg JJ, Gerthoffer WT, Gilbert SH, Gunst SJ, Hai CM, Halayko AJ, Hirst SJ, James AL, Janssen LJ, Jones KA, King GG, Lakser OJ, Lambert RK, Lauzon AM, Lutchen KR, Maksym GN, Meiss RA, Mijailovich SM, Mitchell HW, Mitchell RW, Mitzner W, Murphy TM, Paré PD, Schellenberg RR, Seow CY, Sieck GC, Smith PG, Smolensky AV, Solway J, Stephens NL, Stewart AG, Tang DD, Wang L. On the terminology for describing the length-force relationship and its changes in airway smooth muscle. J.Appl.Physiol. 2004; 97: 6: 2029-2034.

14. Hackett TL, Shaheen F, Johnson A, Wadsworth S, Pechkovsky DV, Jacoby DB, Kicic A, Stick SM, Knight DA. Characterization of side population cells from human airway epithelium. Stem Cells 2008; 26: 10: 2576-2585.

15. Ali F, Chin L, Paré PD, Seow CY. Mechanism of partial adaptation in airway smooth muscle after a step change in length. J.Appl.Physiol. 2007; 103: 2: 569-577.

16. Bossé Y, Chin LY, Paré PD, Seow CY. Adaptation of Airway Smooth Muscle to Basal Tone: Relevance to Airway Hyperresponsiveness. Am.J.Respir.Cell Mol.Biol. 2008.

17. Pratusevich VR, Seow CY, Ford LE. Plasticity in canine airway smooth muscle. J.Gen.Physiol. 1995; 105: 1: 73-94.

18. Seow CY, Pratusevich VR, Ford LE. Series-to-parallel transition in the filament lattice of airway smooth muscle. J.Appl.Physiol. 2000; 89: 3: 869-876.

19. Seow CY and Stephens NL. Force-velocity curves for smooth muscle: analysis of internal factors reducing velocity. Am.J.Physiol. 1986; 251: 3 Pt 1: C362-8.

20. Hill AV. The heat of shortening and the dynamic constants of muscle. 1938; 126: 136195.

21. Syyong H, Cheung C, Solomon D, Seow CY, Kuo KH. Adaptive response of pulmonary arterial smooth muscle to length change. J.Appl.Physiol. 2008; 104: 4: 1014-1020.

22. Herrera AM, Martinez EC, Seow CY. Electron microscopic study of actin polymerization in airway smooth muscle. Am.J.Physiol.Lung Cell.Mol.Physiol. 2004; 286: 6: L1161-8.

23. Kuo KH, Wang L, Paré PD, Ford LE, Seow CY. Myosin thick filament lability 
induced by mechanical strain in airway smooth muscle. J.Appl.Physiol. 2001; 90: 5: 1811-1816.

24. Gunst SJ, Meiss RA, Wu MF, Rowe M. Mechanisms for the mechanical plasticity of tracheal smooth muscle. Am.J.Physiol. 1995; 268: 5 Pt 1: C1267-76.

25. Herrera AM, McParland BE, Bienkowska A, Tait R, Paré PD, Seow CY. 'Sarcomeres' of smooth muscle: functional characteristics and ultrastructural evidence. J.Cell.Sci. 2005; 118: Pt 11: 2381-2392.

26. Dillon PF, Aksoy MO, Driska SP, Murphy RA. Myosin phosphorylation and the cross-bridge cycle in arterial smooth muscle. Science 1981; 211: 4481: 495-497.

27. Mitchell RW, Seow CY, Burdyga T, Maass-Moreno R, Pratusevich VR, Ragozzino J, Ford LE. Relationship between myosin phosphorylation and contractile capability of canine airway smooth muscle. J.Appl.Physiol. 2001; 90: 6: 2460-2465.

28. Paré PD, McParland BE, Seow CY. Structural basis for exaggerated airway narrowing. Can.J.Physiol.Pharmacol. 2007; 85: 7: 653-658.

29. Kuo KH, Herrera AM, Wang L, Paré PD, Ford LE, Stephens NL, Seow CY. Structurefunction correlation in airway smooth muscle adapted to different lengths. Am.J.Physiol.Cell.Physiol. 2003; 285: 2: C384-90.

30. Skloot G, Permutt S, Togias A. Airway hyperresponsiveness in asthma: a problem of limited smooth muscle relaxation with inspiration. J.Clin.Invest. 1995; 96: 5: 2293403.

31. Moore BJ, Verburgt LM, King GG, Paré PD. The effect of deep inspiration on methacholine dose-response curves in normal subjects. Am.J.Respir.Crit.Care.Med. 1997; 156: 4: 1278-81.

32. Schellenberg RR and Foster A. In vitro responses of human asthmatic airway and pulmonary vascular smooth muscle. Int.Arch.Allergy Appl.Immunol. 1984; 75: 3: 237241.

33. Roberts JA, Raeburn D, Rodger IW, Thomson NC. Comparison of in vivo airway responsiveness and in vitro smooth muscle sensitivity to methacholine in man. Thorax 1984; 39: 11: 837-843.

34. Cerrina J, Le Roy Ladurie M, Labat C, Raffestin B, Bayol A, Brink C. Comparison of human bronchial muscle responses to histamine in vivo with histamine and isoproterenol agonists in vitro. Am.Rev.Respir.Dis. 1986; 134: 1: 57-61.

35. Goldie RG, Spina D, Henry PJ, Lulich KM, Paterson JW. In vitro responsiveness of human asthmatic bronchus to carbachol, histamine, beta-adrenoceptor agonists and theophylline. Br.J.Clin.Pharmacol. 1986; 22: 6: 669-676. 
36. de Jongste JC, Mons H, Bonta IL, Kerrebijn KF. In vitro responses of airways from an asthmatic patient. Eur.J.Respir.Dis. 1987; 71: 1: 23-29.

37. Whicker SD, Armour CL, Black JL. Responsiveness of bronchial smooth muscle from asthmatic patients to relaxant and contractile agonists. Pulm.Pharmacol. 1988; 1: 1: 25-31.

38. Cerrina J, Labat C, Haye-Legrande I, Raffestin B, Benveniste J, Brink C. Human isolated bronchial muscle preparations from asthmatic patients: effects of indomethacin and contractile agonists. Prostaglandins 1989; 37: 4: 457-469.

39. Bai TR. Abnormalities in airway smooth muscle in fatal asthma. Am.Rev.Respir.Dis. 1990; 141: 3: 552-557.

40. Bai TR. Abnormalities in airway smooth muscle in fatal asthma. A comparison between trachea and bronchus. Am.Rev.Respir.Dis. 1991; 143: 2: 441-443.

41. Thomson RJ, Bramley AM, Schellenberg RR. Airway muscle stereology: implications for increased shortening in asthma. Am.J.Respir.Crit.Care Med. 1996; 154: 3 Pt 1: 749-757.

42. Bosse Y, Chin LY, Pare PD, Seow CY. Chronic Activation in Shortened Airway Smooth Muscle: A Synergistic Combination Underlying Airway Hyperresponsiveness? Am.J.Respir.Cell Mol.Biol. 2009. 


\section{CHAPTER 5: MECHANICAL PROPERTIES OF ASTHMATIC AIRWAY SMOOTH MUSCLE REVISITED ${ }^{1}$}

\subsection{Introduction}

Asthma is characterized by exaggerated airway narrowing caused by airway smooth muscle (ASM) shortening. However it is unclear whether there is a fundamental phenotypic change in the smooth muscle itself or if the non-muscle components of the airway wall or surrounding lung parenchyma are primary contributors to disease [1]. A major hurdle to a clear understanding of ASM contractile function in disease has been the limited published data. Of the twelve studies that have compared asthmatic and nonasthmatic ASM mechanical properties, seven have demonstrated no differences [2-8], while five have shown increases in force, shortening, or agonist sensitivity [9-13].

We have previously demonstrated that ASM cell bundles carefully dissected from the tracheas of non-asthmatic intact lungs donated for medical research provides a valuable, high quality tissue preparation for mechanical study [14]. Our study demonstrated that the mechanical properties of non-asthmatic ASM were similar to those measured in other mammalian models. This is in contrast to previous studies which suggested that human ASM produced less force per unit area and shortened less than the ASM of other mammals. Importantly, this was the first study to examine human ASM at its in situ length instead of at an arbitrarily determined $\mathrm{L}_{\max }$ starting point. We were able to confirm

\footnotetext{
${ }^{1}$ A version of this chapter will be submitted for publication. Chin LYM, Bossé Y, Solomon D, Hackett TL, Paré PD, Seow CY. (2010) Mechanical properties of asthmatic airway smooth muscle revisited.
} 
for the first time that length adaptation, the time-dependent process by which a reference length in the length-tension relationship of smooth muscle is shifted due to a change in muscle length, does occur in human ASM.

Given the differences between previous findings and our own among non-asthmatics, it would be pertinent to reexamine the mechanical properties of asthmatic ASM, thus, the purpose of this study was to reevaluate whether asthmatic ASM demonstrates altered mechanical properties compared to non-asthmatic ASM. Importantly, the airways were relaxed in zero calcium Krebs solution before determining the in situ length of the muscle. We sought to address a series of hypotheses related to ASM mechanics which have been suggested in the literature as possible defects in asthmatic ASM function and potential contributors to airway hyperresponsiveness (AHR). These include determining whether asthmatic ASM produces more stress (force per unit cross-sectional area of muscle) than non-asthmatic ASM $[15,16]$; whether the length-tension relationship of asthmatic ASM or its ability to undergo length adaptation are altered [17, 18]; whether the muscle shortens faster or more extensively in asthmatics than non-asthmatics [19-22]; and whether asthmatic ASM responds differently to a mechanical perturbation than nonasthmatic ASM [1, 23, 24]. Any of these alterations could potentially impact airway narrowing in response to bronchoconstricting stimuli. 


\subsection{Materials and Methods}

\subsubsection{Tissue Preparation and Equilibration}

Tracheas were removed from non-transplantable human lungs donated for research through the International Institute for the Advancement of Medicine (IIAM: Edison, NJ). Donor deaths were primarily through head trauma among non-asthmatics, while five of seven asthmatics likely died during exacerbations of their disease. The subject demographics and clinical details are shown in Table 1 . The study was approved by the UBC-St. Paul's Hospital Ethics Committee (Appendix A). Whole lungs were obtained as previously described [Chapter 4, 14, 25]. Briefly, after surgical removal the lungs were flushed with Custodiol HTK solution (Odyssey Pharmaceuticals: East Hanover, NJ) and transported by plane on ice to Vancouver. The average transport time was 15-20 $\mathrm{h}$.

The dissection and preparation of the smooth muscle has been previously described [14]. Briefly, upon arrival at the hospital, the tracheal tissue was stored at $4^{\circ} \mathrm{C}$ in physiological saline solution (PSS). Dissection was undertaken within a day. Tracheal rings were placed in zero-Ca ${ }^{2+}$ PSS to relax the muscle in order to accurately determine the in situ resting length of the tracheal smooth muscle (herein referred to as reference length, $\mathrm{L}_{\text {ref }}$ ). Connective tissue and epithelium was carefully removed to isolate a smooth muscle bundle. Muscle strips measuring 1-1.5 mm wide, $0.5 \mathrm{~mm}$ thick, and $6 \mathrm{~mm}$ long, were bound on each end with aluminum foil clips and mounted vertically on a force-length transducer. The muscle was then equilibrated at $\mathrm{L}_{\text {ref }}$ by periodic electrical field stimulation (EFS) at 5-minute intervals for a period of $1.5 \mathrm{~h}$. The CysLT1 receptor 
Table 5.1. Subject demographics and clinical details.

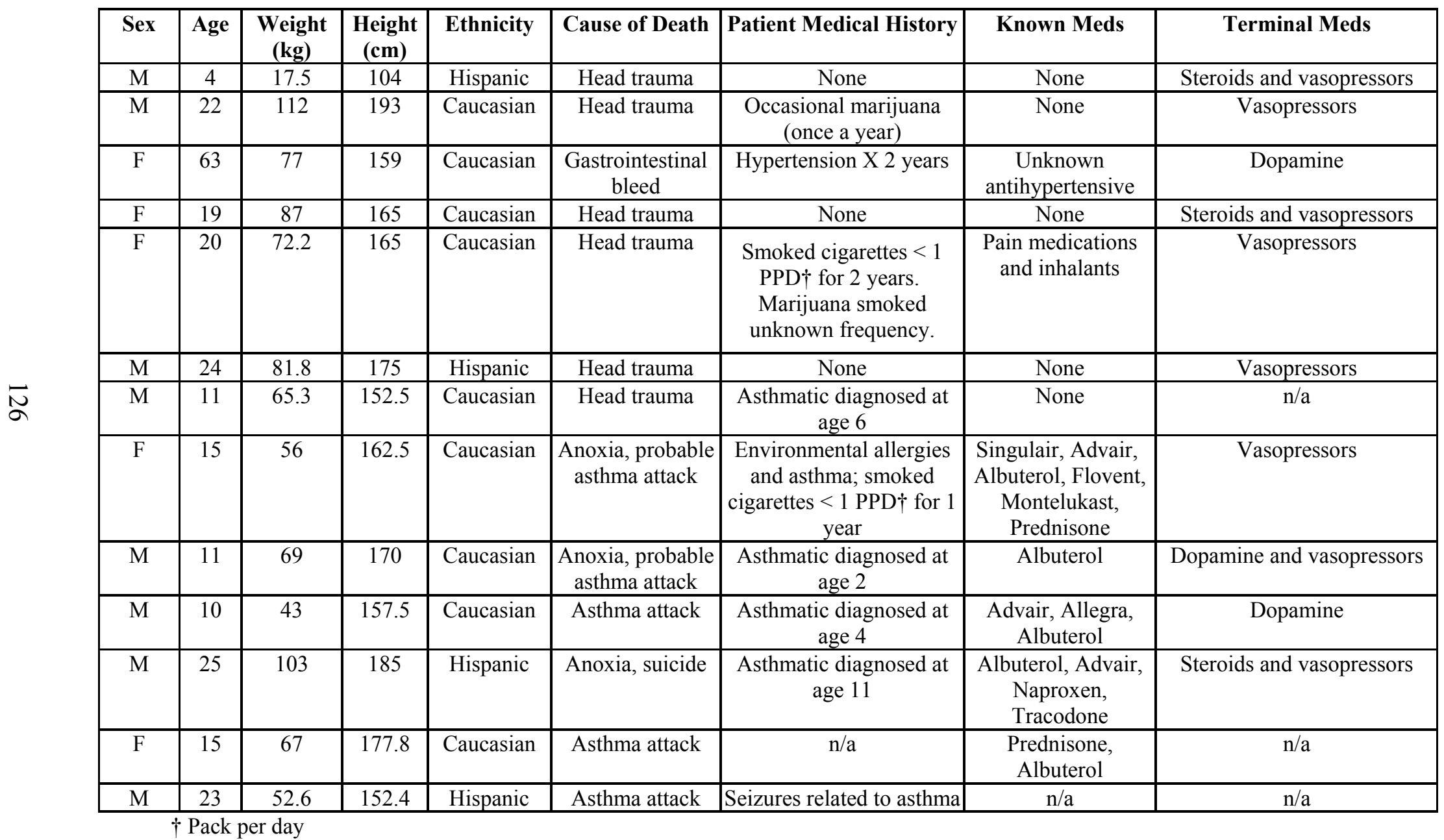


antagonist montelukast $\left(10^{-6} \mathrm{M}\right)$ was added to the PSS for all of the experiments, which prevented or eliminated intrinsic tone.

\subsubsection{Mechanical Measurements}

After equilibration, the maximal isometric force produced in response to EFS at $L_{\text {ref }}$ was determined (herein called $\mathrm{F}_{\max }$ ). As previously described, the force-length relationship was determined at five different lengths: $0.5,0.75,1.0,1.25$, and $1.5 \mathrm{~L}_{\text {ref. }}$ The muscle was allowed to adapt at each length for 20 minutes with EFS at 5-minute intervals. Forcevelocity curves were determined by quick release (quick switch from isometric to isotonic contraction) at five graded loads (between zero and $\mathrm{F}_{\max }$ ) at $\mathrm{L}_{\text {ref. }}$ Velocities were determined at two time points $100 \mathrm{msec}$ after release, as previously described: at the peak of tetanic force and midway to the peak $[14,26]$. The curves were fit using AV Hill's hyperbolic equation [27]. Maximal isotonic shortening was determined by extrapolation from two low-load isotonic EFS contractions, at preloads of $10 \%$ and $20 \% \mathrm{~F}_{\max }$. Since the

relationship between load and shortening is assumed to be linear [28], maximal shortening at zero-load was extrapolated from the two points. Response to mechanical perturbation was determined by applying a passive 10 minute, $0.2 \mathrm{~Hz}, 30 \% \mathrm{~L}_{\text {ref }}$ length oscillation. Force recovery following oscillation was followed for 30 minutes with EFS at 5-minute intervals.

\subsubsection{Histology}

At the end of the mechanical measurements the tissue was fixed at $\mathrm{L}_{\text {ref }}$ in $10 \%$ formalin for histology. The histology was performed as previously described [14]. The amount of 
muscle in the preparation was determined by staining transverse sections with Masson's trichrome and quantified by tracing using Image ProPlus 4.5 (MediaCybernetics: Bethesda, MD). Connective tissue embedded within muscle bundles was excluded by color segmentation. Maximal stress generating capacity was determined by dividing $\mathrm{F}_{\max }$ (in $\mathrm{mN}$ ) by the cross-sectional area (in $\mathrm{mm}^{2}$ ) of muscle present in the preparation.

\subsubsection{Statistical Analysis}

Force and length measurements were normalized to $\mathrm{F}_{\max }$ or $\mathrm{L}_{\text {ref }}$ respectively and expressed as fractions of $\mathrm{F}_{\max }$ and $\mathrm{L}_{\text {ref. }}$. Velocity of shortening was expressed as $\mathrm{L}_{\text {ref }} / \mathrm{sec}$. Aggregate data were expressed as mean \pm SEM. ANOVA and regression analyses were accomplished using GraphPad Prism 5 (GraphPad Software, Inc.: La Jolla, CA). $\mathrm{p} \leq 0.05$ was considered to be sufficient to reject the null hypothesis.

\section{$5.3 \quad$ Results}

\subsubsection{Muscle Bundle Properties}

Six non-asthmatic and seven asthmatic lungs were received from donors (Table 1). The average age of the non-asthmatics and asthmatics were $25.3 \pm 8.1$ years and $15.7 \pm 2.3$ years respectively (t-test: $\mathrm{p}=0.247$ ). Muscle strips were fixed in formalin and stained with Masson's Trichrome for histological morphometry (non-asthmatics: $\mathrm{n}=4$, asthmatics: $\mathrm{n}=8$ ). The non-asthmatics generated an average maximal stress of $88.45 \pm 18.8 \mathrm{mN} / \mathrm{mm}^{2}$ while the asthmatics generated $130.2 \pm 32.6 \mathrm{mN} / \mathrm{mm}^{2}$ (Fig. 1B). The two groups were not statistically different (Student's t-test: $\mathrm{p}=0.414$ ). 


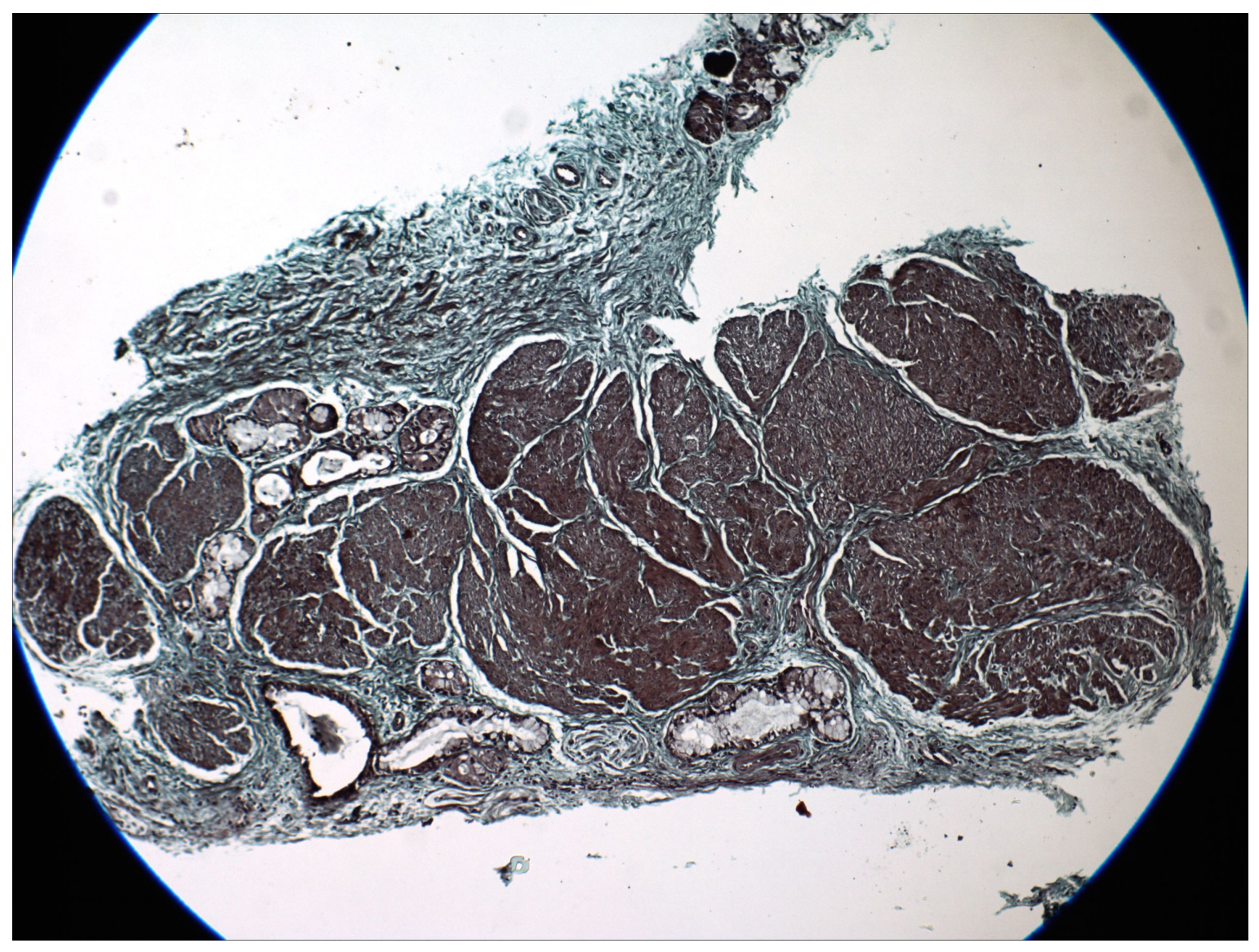

Figure 5.1 Asthmatic airway smooth muscle morphology. ASM strips stained with Masson's Trichrome were used to calculate the maximal stress (force per unit area muscle) and the proportion of muscle per dissected preparation. The smooth muscle is red, connective tissue blue, and nuclei black. Color segmentation was used to exclude the blue connective tissue within muscle bundles. Image width $=1.14 \mathrm{~mm}$. 


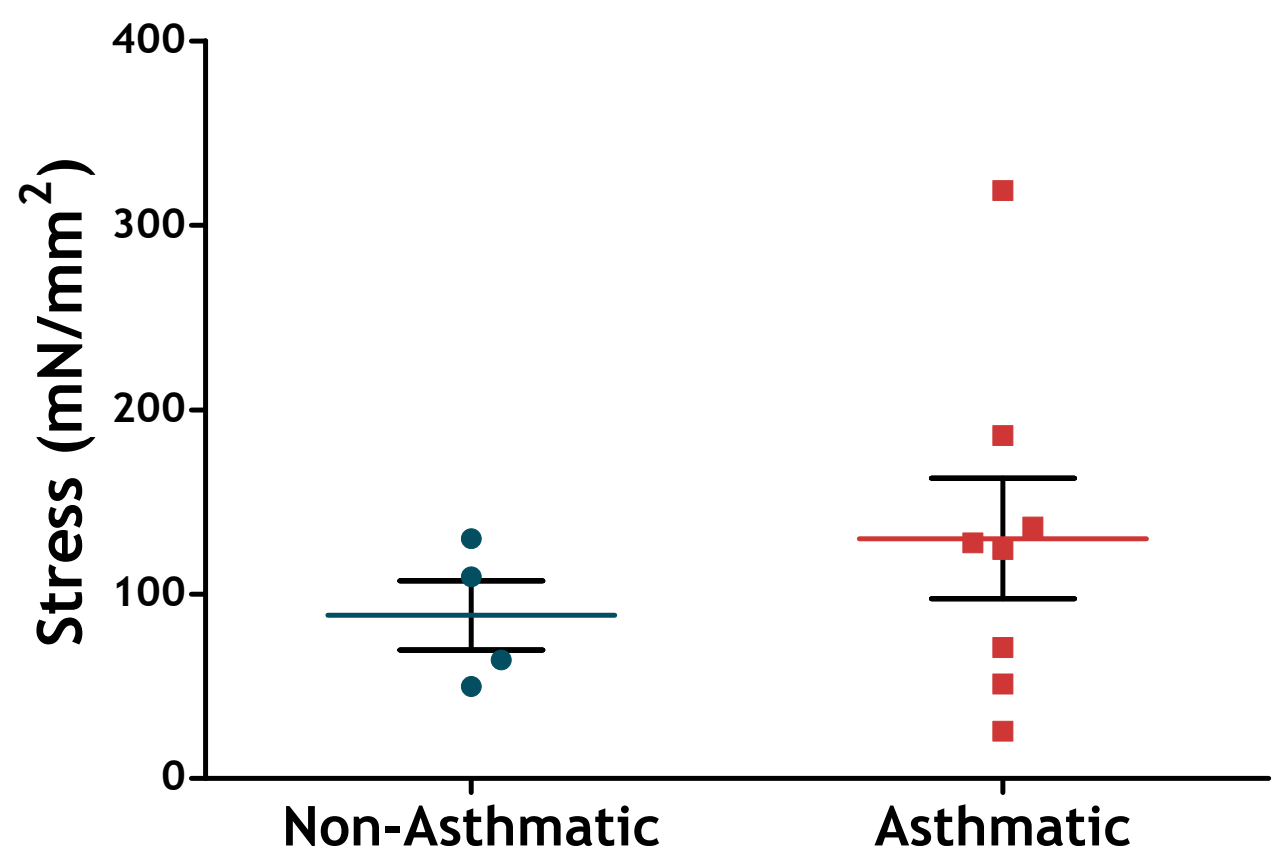

Figure 5.2 Comparison of maximal stress generation. Maximal stress, calculated as $F_{\max }$ divided by muscle cross-sectional area $\left(\mathrm{mN} / \mathrm{mm}^{2}\right)$, between non-asthmatics and asthmatics was compared. Error bars indicate SEM, $n=4$ for non-asthmatics, $n=8$ for asthmatics.

\subsubsection{Length-Force Properties and Length Adaptation}

Length-force properties were examined by recording force at five different lengths: 0.50 , $0.75,1.00,1.25,1.50 \mathrm{~L}_{\text {ref }}$ (Fig. 2). After each length change to a shorter or longer length than $\mathrm{L}_{\text {ref, }}$, the muscle was returned to $\mathrm{L}_{\text {ref }}$ to allow for a period of equilibration or adaptation (Fig. 2A). Immediately following a length change the active force produced by the muscle declined and gradually recovered over the 20 -minute period in which the muscle length was held constant (Fig. 2B). Both groups were corrected for force 
deterioration, as previously explained [14]. Except for the asthmatic group at $1.25 \mathrm{~L}_{\text {ref }}$, force did not recover to the level of $\mathrm{F}_{\max }$ at any of the length steps. Conversely, the passive force increased dramatically after length changes to longer lengths and decreased immediately following a change to a shorter length (Fig. 2C). While the passive force at longer lengths declined over the 20-minute period, it remained elevated across both groups when compared to the passive force at $\mathrm{L}_{\text {ref. }}$.

To determine the extent of length adaptation, the first contraction after a length change was compared to the last contraction at that length (Fig. 3). For both the non-asthmatics and the asthmatics, significant length adaptation occurred (ANOVA: p<0.0001) (Fig. 3A). Bonferroni post-tests revealed significantly greater force at $0.5 \mathrm{~L}_{\text {ref }}$ and $1.5 \mathrm{~L}_{\text {ref }}$ among the non-asthmatics following adaptation ( $\mathrm{p}<0.05$ and $\mathrm{p}<0.001$, respectively). The asthmatics demonstrated greater active force following adaptation at 1.25 and $1.50 \mathrm{~L}_{\text {ref }}(\mathrm{p}<0.001)$. The active force-length relationship of the asthmatics was similar to the non-asthmatics before the adaptation period (ANOVA: $\mathrm{p}=0.29$ ), with post-tests demonstrating that active force at $1.50 \mathrm{~L}_{\text {ref }}$ was significantly lower in asthmatics compared to non-asthmatics $(\mathrm{p}=0.05)$. After the adaptation period the asthmatics produced less force than the adapted non-asthmatics at $0.50 \quad \mathrm{~L}_{\text {ref }}(\mathrm{p}<0.01)$. Both the non-asthmatics and asthmatics demonstrated significant passive force adaptation - i.e. the passive force decreased significantly after a period of adaptation compared among the non-asthmatics or asthmatics (ANOVA: $\mathrm{p}=0.024$ for non-asthmatics and $\mathrm{p}=0.018$ for asthmatics). Bonferroni post-tests demonstrate that the passive force at $1.50 \mathrm{~L}_{\text {ref }}$ for the asthmatics was significantly greater than that of the non-asthmatics $(\mathrm{p}<0.05)$. The passive force at 1.50 
Lref was also significantly lower in the asthmatics after adaptation than before adaptation $(\mathrm{p}<0.05)$ 
Figure 5.3 Time course of length changes and the resulting active and passive force. ASM strips were either shortened or lengthened in the relaxed state to $0.50,0.75,1.25$ or $1.50 \mathrm{~L}_{\text {ref. }}$ The muscle was stimulated to contract 5 times with EFS at 5-minute intervals after every length change. The force was corrected for force deterioration over time (refer to text for details). Between shortening and lengthening steps, the muscle was returned to $\mathrm{L}_{\text {ref }}$ and readapted. Grey hexagons indicate the time points where EFS occurred (A). The active force (B) and passive force (C) were recorded at every contraction. Both were normalized to $F_{\max }$. Non-asthmatics are represented as black circles, while asthmatics are represented as grey squares. Error bars indicate SEM, $\mathrm{n}=6$ for each group. 


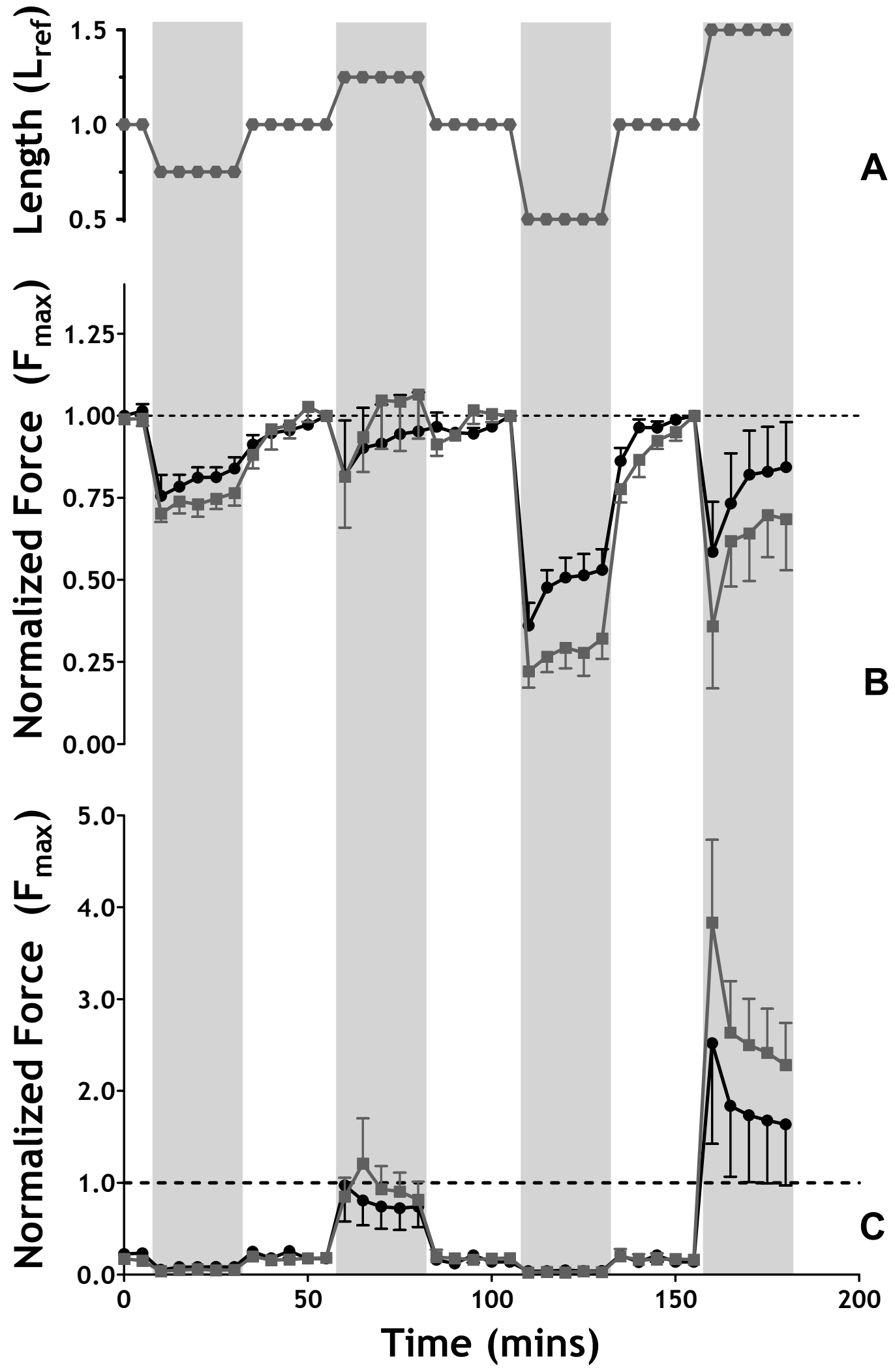




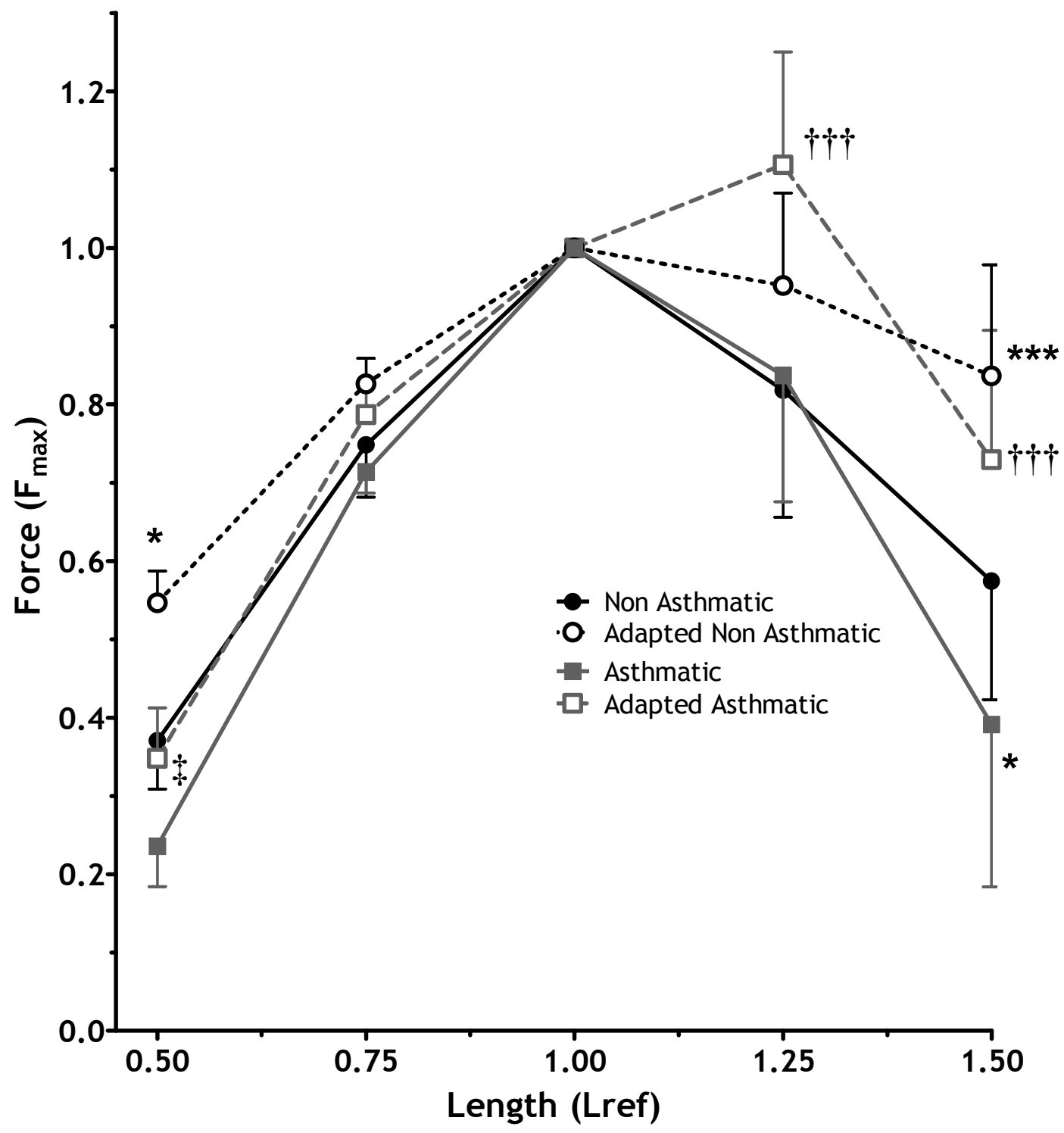

Figure 5.4 Active force-length relationship before and after length adaptation. From $\mathrm{L}_{\text {ref, }}$ the ASM strips were either shortened or lengthened to $0.50,0.75,1.25$, and $1.50 \mathrm{~L}_{\text {ref }}$ (refer to Fig. 5.3 for sequence and time course of length changes and stimulation). Active force before (solid lines and closed symbols) and after (dashed lines and open symbols) length adaptation are shown. Force was corrected for deterioration over the time period of the experiment, see text for details. Non-asthmatics are represented by black circles and 
asthmatics by gray squares. Error bars indicate SEM, $\mathrm{n}=6$ for each group. *: $\mathrm{p}<0.05$, ***: $\mathrm{p}<0.001$; compared to the non-asthmatic before length adaptation at the given length. $\uparrow \uparrow \uparrow$ : $\mathrm{p}<0.001$; compared to the asthmatic before length adaptation at the given length. $f$ : $\mathrm{p}<0.05$; compared to the non-asthmatic after length adaptation at the given length.

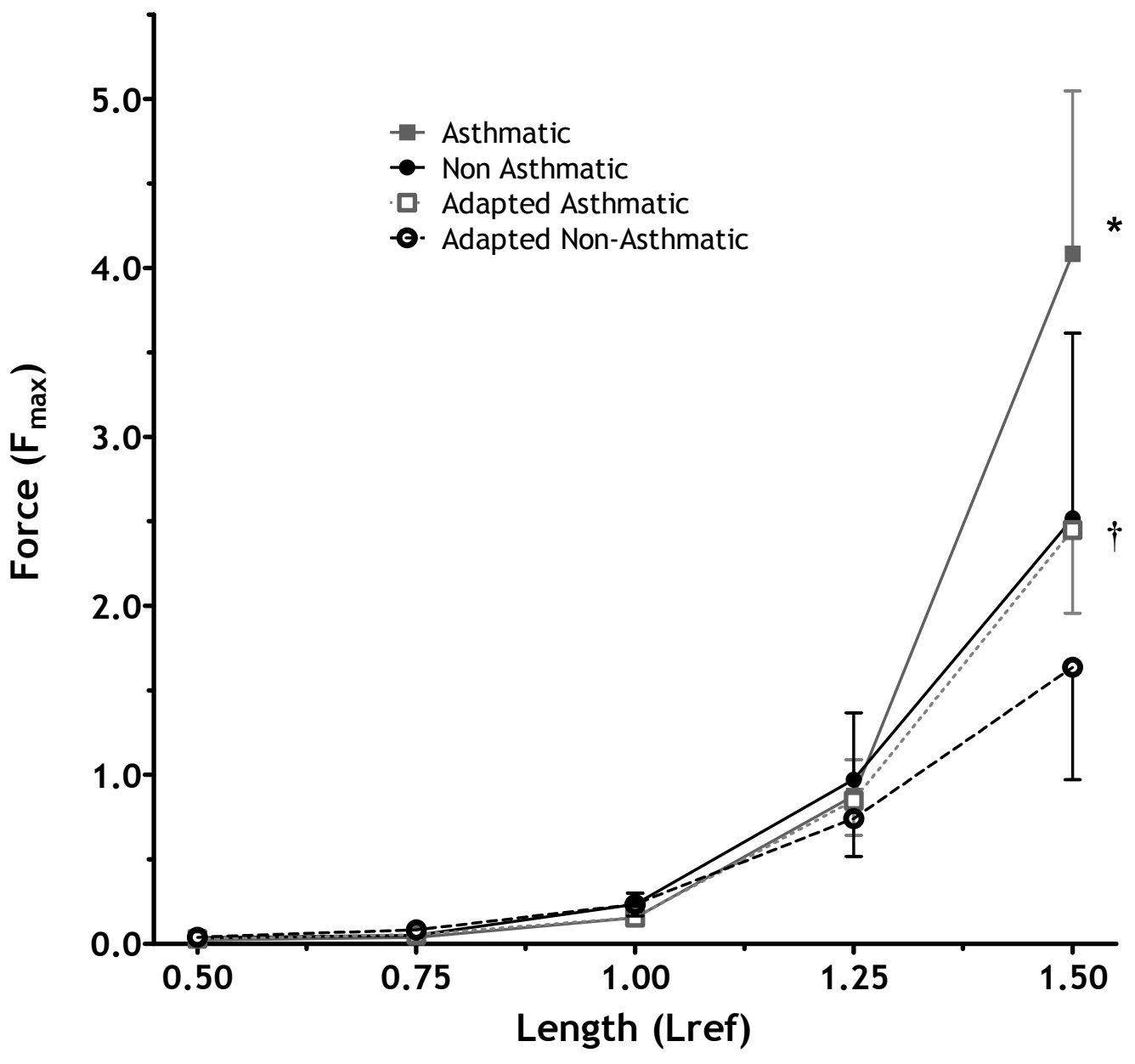

Figure 5.5 Passive force-length relationship before and after length adaptation. From $\mathrm{L}_{\text {ref, }}$ the ASM strips were either shortened or lengthened to $0.50,0.75,1.25$, and $1.50 \mathrm{~L}_{\text {ref }}$ (as explained above). Passive force both before (solid lines and closed symbols) and after (dashed lines and open symbols) length adaptation are shown. Non-asthmatics are 
represented by black circles and asthmatics by gray squares. Error bars indicate SEM, n=6 for each group. *: $\mathrm{p}<0.05$; compared to the non-asthmatic before length adaptation at the given length. $\uparrow: \mathrm{p}<0.05$; compared to the asthmatic before length adaptation at the given length.

\subsubsection{Shortening Velocity}

Two sets of force-velocity relationships were determined: one during the early-phase of contraction and one during the peak of tetanic contraction, as previously explained [14]. The force-velocity curves for individual muscle strips varied widely (Fig. 4A \& 4B). Force-velocity data for each muscle strip was initially fitted with Hill's hyperbolic equation before averaging the curves (Fig. 4C \& 4D). At the early-phase of contraction (Fig. 4C) and the peak of tetanic contraction (Fig. 4D), the force-velocity curves between asthmatics and non-asthmatics were not significantly different. 


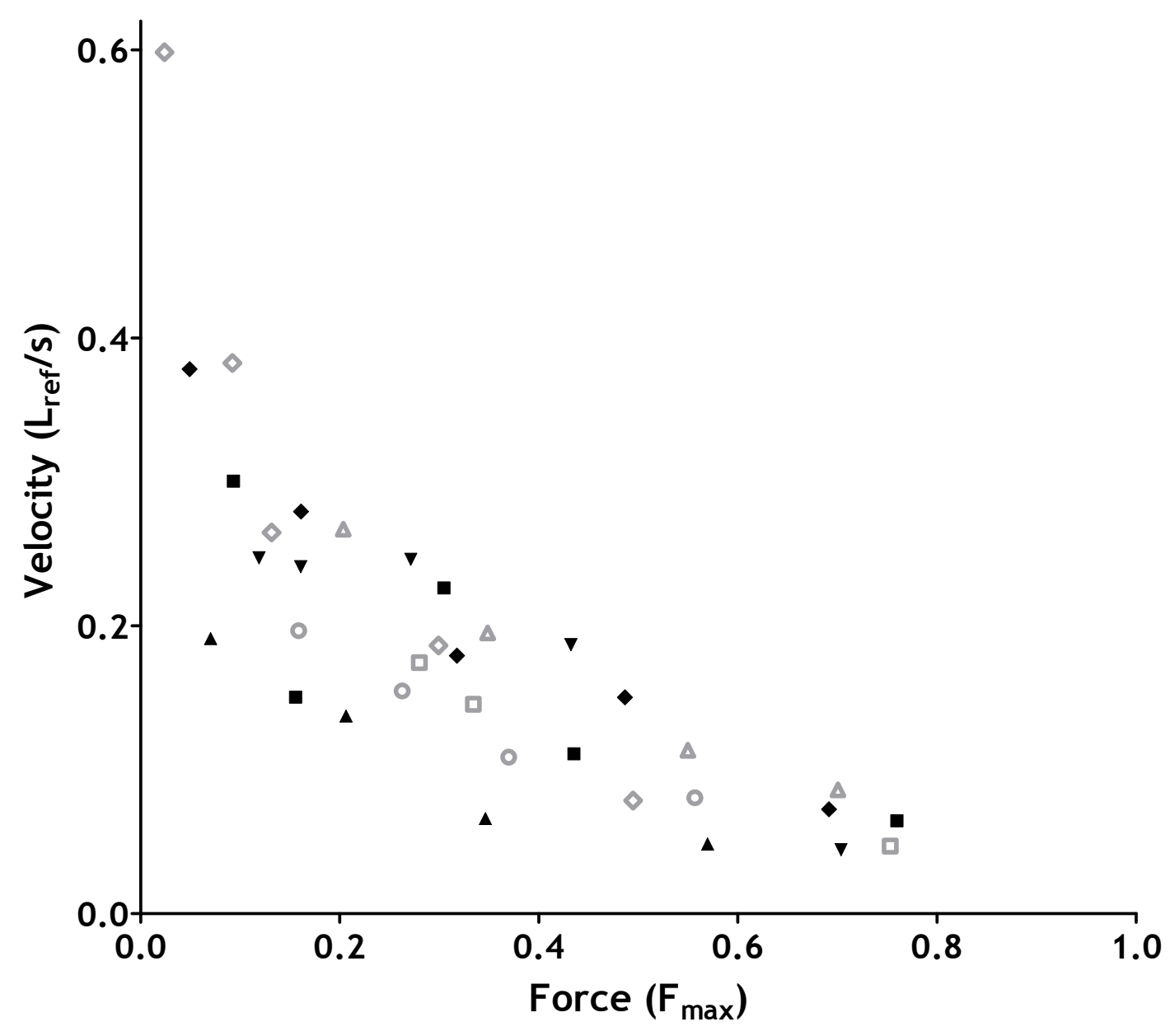

Figure 5.6 Isotonic shortening velocity, individual data points at the early release. Isotonic shortening velocity was measured at different loads the early phase of contraction (refer to methods for details). The loads were determined as a percentage of $\mathrm{F}_{\max }$. Individual velocity data points recorded at different loads are shown. Every different symbol represents a unique muscle strip. Non-asthmatics are represented as black solid symbols and asthmatics as gray open symbols. Data points were fitted with Hill's hyperbolic equation and the subsequent curves were averaged to generate the Fig. 5.8. $n=4$ for each group. 


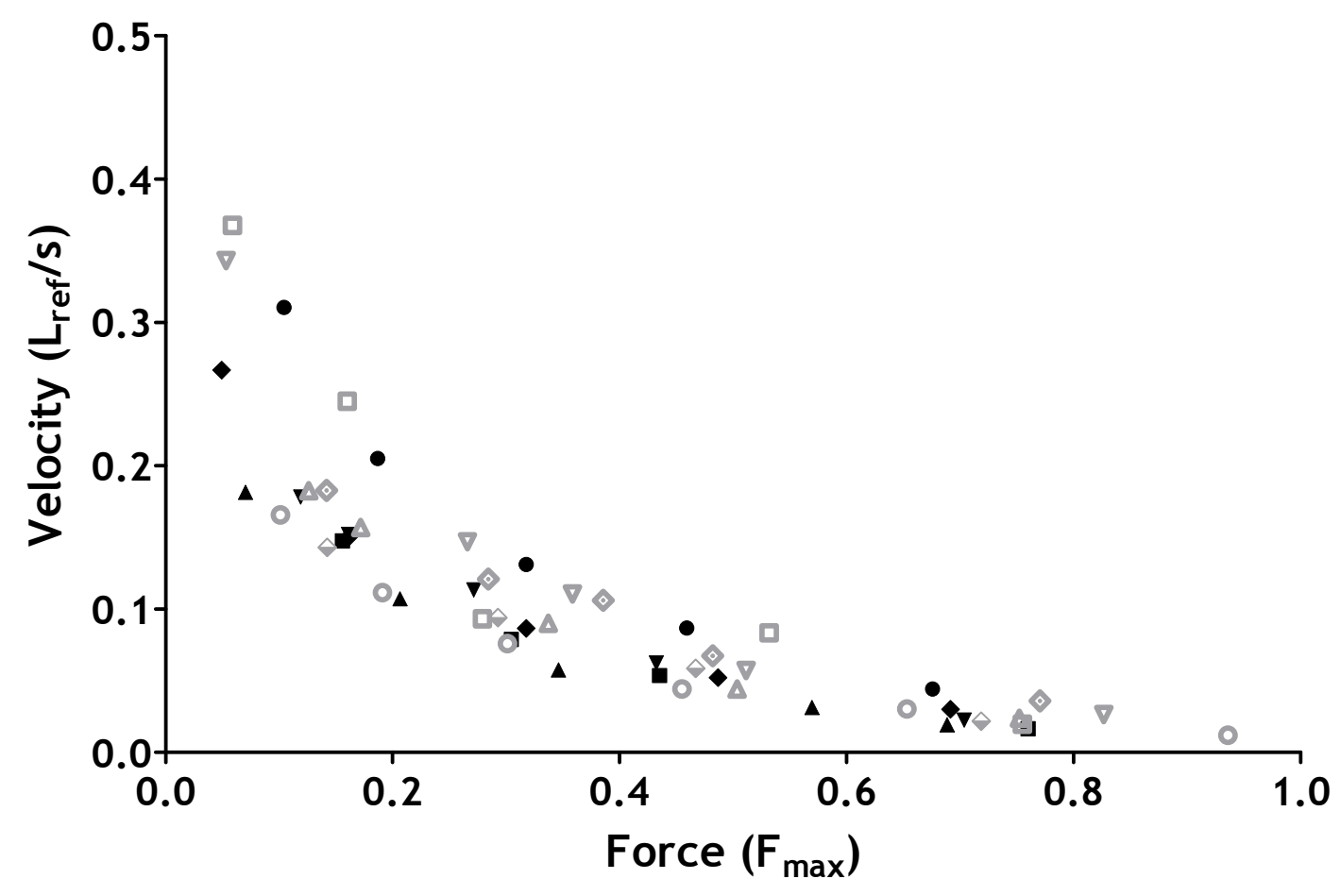

Figure 5.7 Isotonic shortening velocity, individual data points at the late release. Isotonic shortening velocity was measured at different loads at the late phase of contraction (refer to methods for details). The loads were determined as a percentage of $\mathrm{F}_{\max }$. Individual velocity data points recorded at different loads are shown. Every different symbol represents a unique muscle strip. Non-asthmatics are represented as black solid symbols and asthmatics as gray open symbols. Data points were fitted with Hill's hyperbolic equation and the subsequent curves were averaged to generate Fig. 5.9. $\mathrm{n}=5$ for the non-asthmatics and $n=6$ for the asthmatics. 


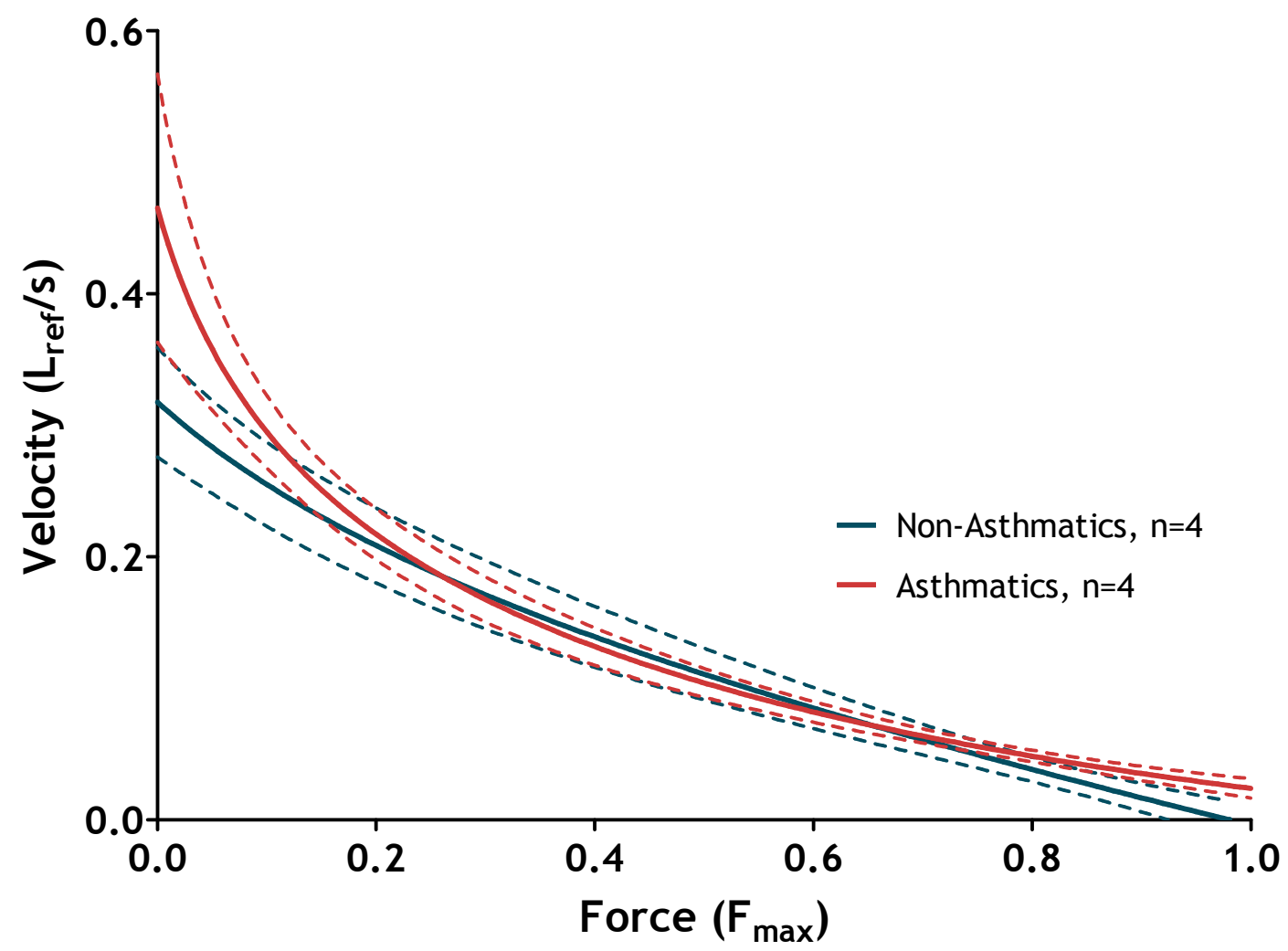

Figure 5.8 Averaged isotonic shortening velocities to various loads, early phase release. Hill's hyperbolic equation was fitted to each sample in Fig. 5.6. The solid lines represent the average of these curves. The dashed lines indicate SEM. 


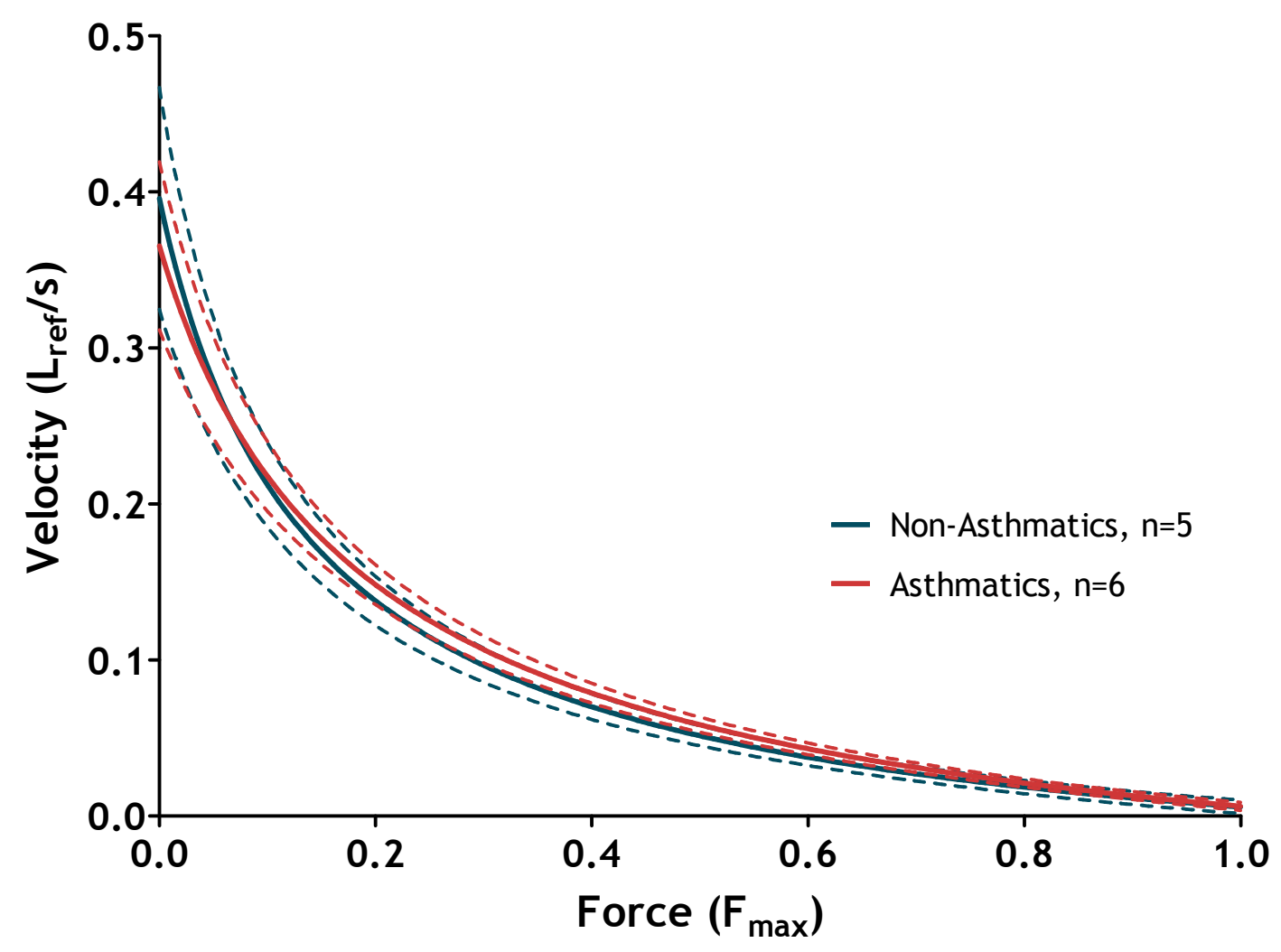

Figure 5.9 Averaged isotonic shortening velocities to various loads, late phase release. Hill's hyperbolic equation was fitted to each sample in Fig. 5.7. The solid lines represent the average of these curves. The dashed lines indicate SEM.

\subsubsection{Maximal Isotonic Shortening}

Four non-asthmatic and four asthmatic muscle strips were analyzed to determine maximal isotonic shortening (Fig. 5.10). The extent of shortening was recorded at $10 \%$ and $20 \%$ $\mathrm{F}_{\max }$ loads. Maximal shortening, as determined by extrapolation, was $72.2 \pm 4.9 \%$ of the total muscle strip among the non-asthmatics and $70.5 \pm 5.6 \%$ for the asthmatics (nonasthmatics regression: $p=0.006, r^{2}=0.749$; asthmatics regression: $\left.p=0.013, r^{2}=0.6725\right)$. The two groups were not statistically different $(p=0.946)$. With no difference between the 
groups, all muscle strips were combined into one linear regression (central dashed line, Fig. 5), with maximal shortening determined as $71.4 \pm 3.4 \%\left(\mathrm{p}<0.0001, \mathrm{r}^{2}=0.708\right)$. At zeroload the $95 \%$ confidence intervals ranged from 64.0 to $78.8 \%$ shortening.

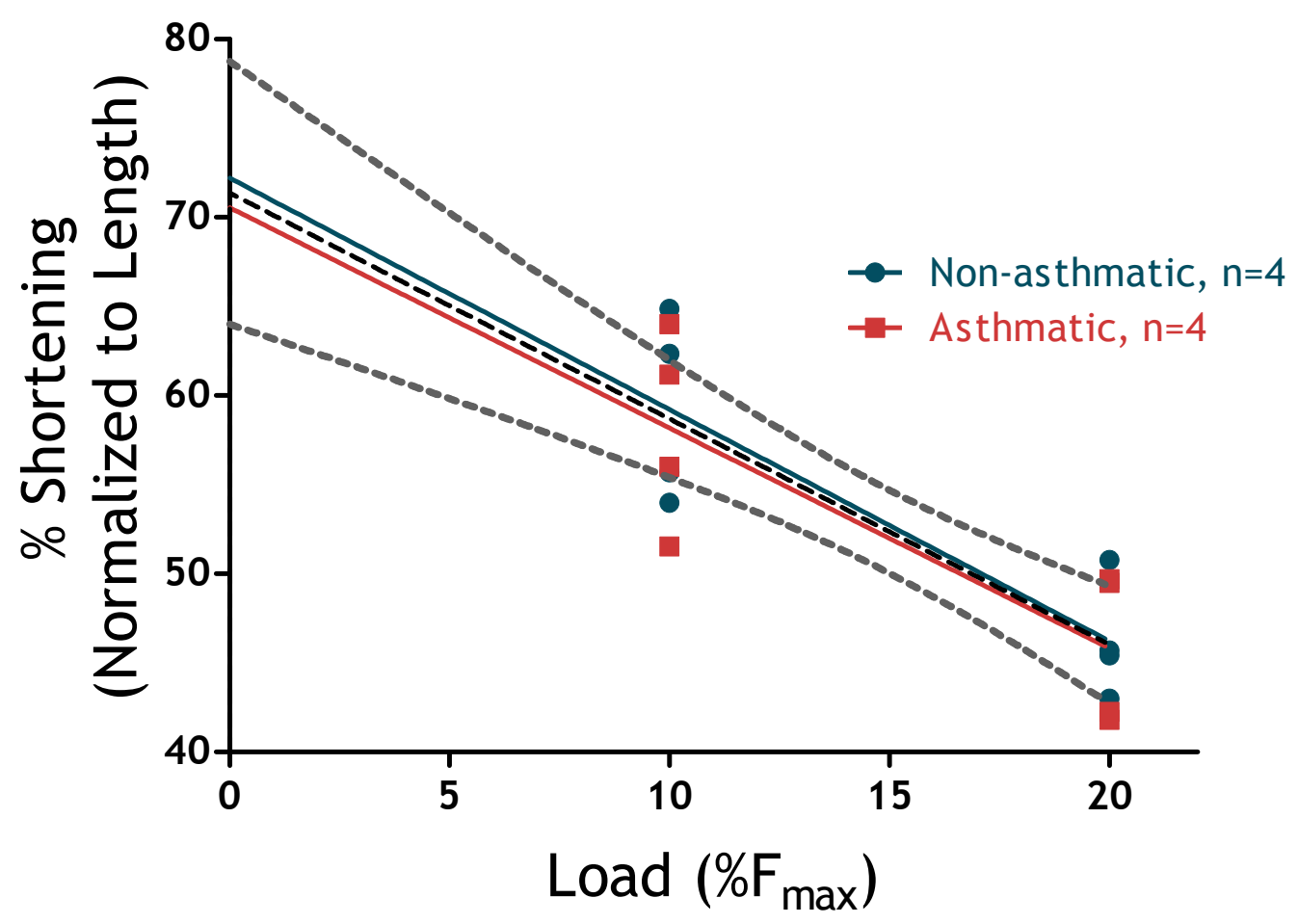

Figure 5.10 Maximal isotonic shortening. ASM strips were stimulated to contract with EFS and the total amount of isotonic shortening against low loads was measured. Using the amount of shortening recorded at loads representing $10 \%$ and $20 \%$ of $\mathrm{F}_{\max }$, maximal shortening was extrapolated. Shortening was recorded at both loads per donor. $\mathrm{n}=4$ for each group. Non-asthmatics are represented as solid circles and asthmatics as solid squares. A combined (both groups) linear regression is represented as a dashed central line. The upper and lower dashed lines represents the $95 \%$ confidence interval for all data points. 


\subsubsection{Recovery from Mechanical Perturbation}

Active force recovery following a ten-minute length oscillation was followed for 30 minutes in both non-asthmatics and asthmatics (Fig. 5.11). The response to oscillation was significantly different between asthmatics and non-asthmatics (ANOVA: $p<0.0001$ ). Initially following oscillation the non-asthmatics produced an average force of $0.643 \pm 0.03$ $\mathrm{F}_{\max }$ compared to $0.807 \pm 0.04 \mathrm{~F}_{\max }$ in asthmatics (Bonferroni post-tests: $\mathrm{p}<0.01$ ). The recovery of force was greatest over the first five minutes in the non-asthmatics, however, the average force recovery was below $F_{\max }$ at $0.953 \pm 0.03 \quad F_{\max }$. The asthmatics demonstrated slower force recovery but recovered beyond $F_{\max }$ after 30-minutes, $1.05 \pm 0.03 \mathrm{~F}_{\max }$. Non-linear regressions of the non-asthmatic and asthmatic data demonstrated these differences in recovery rate. The regressions were fit using a onephase exponential equation, for the non-asthmatics: force $=0.947-0.296 \mathrm{e}^{-0.139 t}$ $\left(\mathrm{r}^{2}=0.725\right)$; asthmatics: force $=1.053-0.243 e^{-0.073 t}$, where force was relative to $\mathrm{F}_{\max }$ and $t$ was time in minutes $\left(\mathrm{r}^{2}=0.481\right)$. 


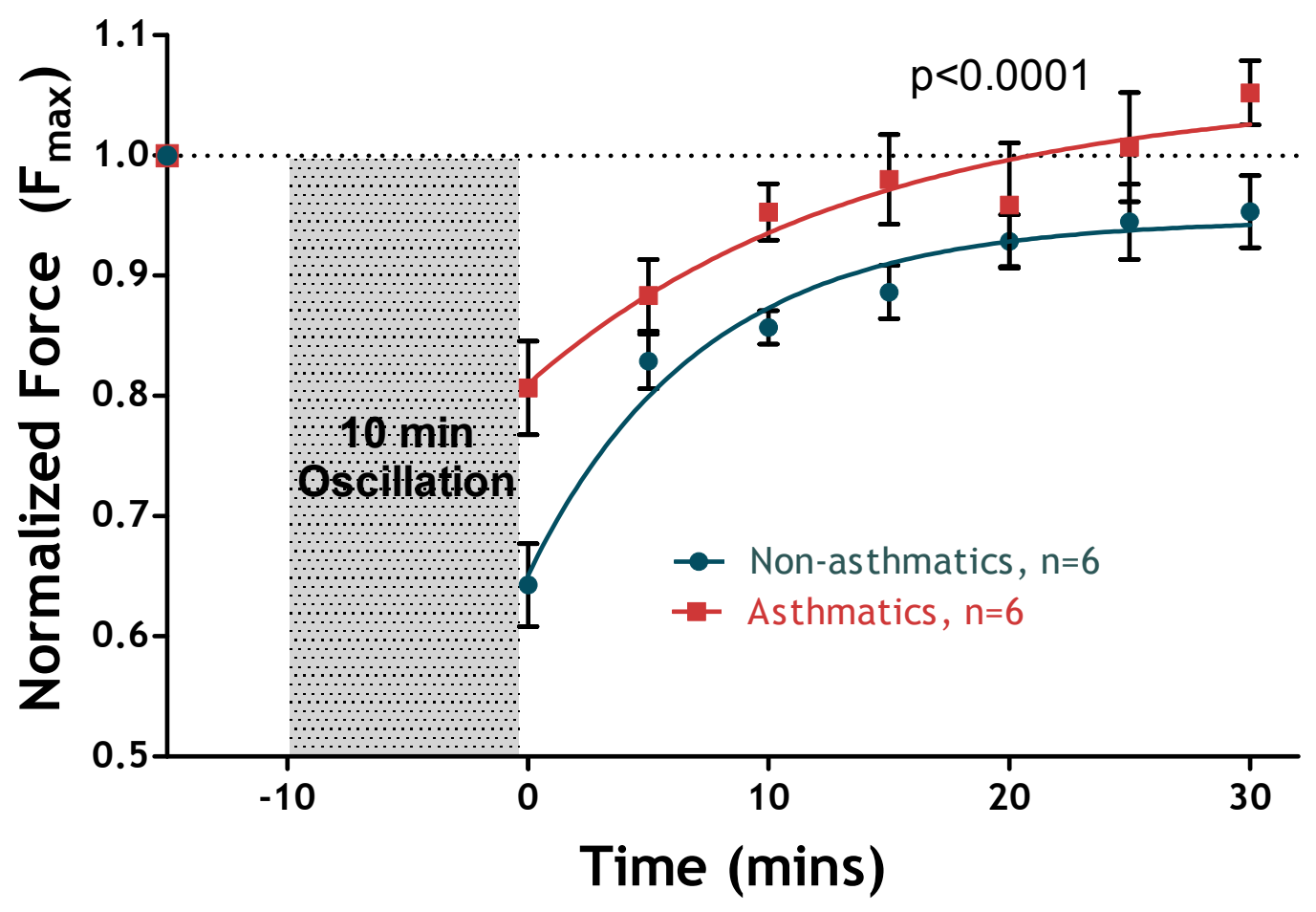

Figure 5.11 Response to mechanical perturbation. Recovery of isometric force following a ten-minute, $0.2 \mathrm{~Hz}, 30 \% \mathrm{~L}_{\text {ref }}$ length oscillations. ASM strips were adapted to $\mathrm{L}_{\text {ref }}$ prior to oscillation. The force produced by seven EFS-induced contractions were recorded at 5-minute intervals following the oscillation. ANOVA: $\mathrm{p}<0.0001$. Error bars indicate SEM, $\mathrm{n}=6$ per group.

\subsection{Discussion}

In this study we sought to examine whether certain mechanical properties of asthmatic ASM could contribute to the clinical features of airway hyperresponsiveness presented by asthmatic patients. While previous studies have attempted to determine whether asthmatic 
ASM is mechanically different than non-asthmatic ASM [2-13], the results thus far have been equivocal. This study provides valuable data on the mechanical properties of asthmatic ASM, and for the first time the mechanical properties were determined within the paradigm of ASM mechanical plasticity [29]. The use of a high quality tissue source also provides a new perspective [14]. From our data it is clear that asthmatic ASM mechanical properties are in fact different, at least in some ways, than non-asthmatic ASM and these differences carry important pathophysiological ramifications.

\subsubsection{Does Asthmatic ASM Produce More Force?}

Perhaps the simplest and most widely suggested possible difference between asthmatics and non-asthmatics is the ability to generate force. However, there is little evidence to support the hypothesis that asthmatic ASM produces more force [30]. Few studies have measured force generated in asthmatics and far fewer have measured the area of the smooth muscle in order to normalize for the size of the muscle strip. Our data suggests that asthmatic ASM produces the same stress (force per unit muscle area) as nonasthmatic ASM (Fig. 1B). Given the wide range of stress produced in each group a posthoc power analysis determined that 46 donors per group would be required to reach statistical significance. While statistical significance may be possible with a larger sample size it is not certain whether the difference in stress production would be physiologically relevant. 


\subsubsection{Is the Force-Length Relationship Different in Asthmatics?}

Using in situ length as our reference length $\left(\mathrm{L}_{\text {ref }}\right)$, both the asthmatics and the nonasthmatics have similar length-force relationships. Although the asthmatic ASM seems to produce less force at the shorter and longer lengths than the non-asthmatics these differences were not significant (Fig. 3A). Even following length adaptation, which is prominently exhibited in both groups, the asthmatic ASM produces less force than the non-asthmatic ASM at a shortened length $\left(0.50 \mathrm{~L}_{\mathrm{ref}}\right)$. This observation demonstrates that asthmatic ASM is no more capable of length adapting than non-asthmatic ASM, and if length adaptation in asthmatic airways indeed contributes to a vicious cycle of airway narrowing as suggested $[1,17,18,31]$, factors outside of the ASM such as the elastic loads of the lung parenchyma must play a role. Our data suggests that this difference in adaptation could actually be protective to the asthmatic airway since the muscle at the shorter length has a reduced force generating capacity. While this may be true, the adapted length-force relationship of asthmatic ASM seems to shift to the right. Thus, at longer lengths $\left(1.25 \mathrm{~L}_{\text {ref }}\right.$ for instance) the process of length adaptation could increase force capacity creating a potential impediment to airway expansion since any tendency for muscle lengthening would be countered by an increase in muscle force (beyond $\mathrm{F}_{\max }$, Fig. $3 \mathrm{~A})$.

\subsubsection{Does Asthmatic ASM Shorten Faster or More Than Non-asthmatic ASM?}

Another prevailing hypothesis that has been suggested to explain airway hyperresponsiveness is altered shortening velocity. This hypothesis has been supported by 
the findings of Jiang et al. [32], in which sensitized canine bronchial smooth muscle was found to shorten faster than non-sensitized muscle and contained more myosin light chain kinase (MLCK). These results were further supported by a later study employing bronchial tissue from asthmatics removed by biopsy [21]. The asthmatic bronchial smooth muscle was found to shorten faster and more extensively than non-asthmatic smooth muscle and contain more MLCK mRNA. In another study, sensitized human airways were found to have greater MLCK and myosin heavy chain (MHC) content [33]. Our data suggests that differences in shortening velocity may not be an important contributor to AHR. There were no statistical differences either at the early-phase or late-phase determinations of shortening velocity (Fig. 4C \& 4D). While the asthmatics' forcevelocity curve generated during early-phase release suggests a potentially higher maximal shortening velocity, the Hill curve may be skewed by a single low-force data point (Fig. 4A). The data are unfortunately limited in the low load region. To accurately survey any differences in maximal shortening velocity we would require more data in the region of force less than $0.05 \mathrm{~F}_{\max }$. This limitation was a technical matter, since the force transducer was unable to reliably determine velocities at forces below $2 \mathrm{mN}$. In the future, dissection of larger strips may circumvent this limitation, such that $\mathrm{F}_{\max }$ would be large enough to avoid loads below $2 \mathrm{mN}$ while working in the $0.05 \mathrm{~F}_{\max }$ range. Regardless, it may be suggested from the late-phase release and the extent of shortening that any differences in shortening may be unlikely contributors to AHR.

Our results provide some important clarity on the previously mentioned studies. It is difficult to compare sensitized canine tissue with asthmatic tissue, thus, the results of the 
Ma et al. study [21] suggest an intriguing dichotomy. It is likely that the differences seen between that study and our own are methodological. In the study, single smooth muscle cells were isolated from bronchial biopsy samples and stimulated to contract under no load. Importantly, the cells were not held at a given length but assumed to be relaxed in the absence of a stimulus. Also, all of the contractions were recorded at room temperature. As previously mentioned, all of our measurements were made at $37^{\circ} \mathrm{C}$ and the intact muscle strips were held at in situ length. It is surprising that the degree of shortening ranged from $25-40 \%$ in Ma et al. [21], similar to previous human mechanical studies in which the muscle was held at $L_{\max }[14,34,35]$, since our muscle strips consistently shorten to about $70 \%$ of the muscle strip when unloaded.

While we have not measured the content of MLCK or MHC in the muscle, it a likely corollary from our data that we would find no differences. Regardless, it would be an important additional piece of information. Ammit et al. [33] found increases in both MLCK and MHC in sensitized human ASM. In the study, smooth muscle bundles were removed from human lung tissue unsuitable for transplantation and some from donors. Of the ten subjects, four had cystic fibrosis, three had alpha-1 antitrypsin deficiency or emphysema, one had primary pulmonary hypertension, and two were healthy lung donors. The muscle bundles were tested to determine their sensitization status by exposure to four common allergen extracts. A muscle preparation was considered 'sensitized' if any of the allergen extracts caused a contraction, while, they were deemed 'unsensitized' if there was no contraction, but a response to acetylcholine. Since no other mechanical measurements were made, such as force generating capacity or shortening velocity, it is unclear whether 
these differences in MLCK and MHC correlate to any functional changes. Also, there is uncertainly with correlating sensitization status of the airways with asthmatic status.

\subsubsection{Does Asthmatic ASM Respond Differently to Mechanical Perturbation?}

This response is perhaps the most intriguing aspect of this study (Fig. 6). It is well known that asthmatics respond differently than non-asthmatics to deep inspirations [24, 36-39]. While the protocol used is certainly extreme when considering that individuals often only take deep breaths every five minutes or so during tidal breathing, the results of this extreme protocol suggest that the impaired bronchodilating response to deep inspirations seen in asthmatics may be an innate property of the ASM itself, independent of the previously hypothesized differences in the asthmatic lung parenchyma or non-contractile elements of the airway wall [31, 40-42]. One possible reason for this innate difference may be due to the increased stiffness seen in asthmatics as suggested from the passive length-force relationship (Fig. 3B). This difference could be due to increased connective tissue surrounding the ASM $[9,43]$. While our muscle strips contained similar muscle area to total dissected area proportions across both groups (not shown), it was not possible to accurately determine whether connective tissue within muscle bundles was increased in the asthmatic group.

The protocol for length oscillation was initially adopted from a study in porcine tissue examining the ability of myosin filaments within ASM cells to reform following mechanical perturbation [44]. Currently, it is impossible to determine whether the 
increased stiffness, perhaps from increased connective tissue, provides a "protective buffer" to the contractile elements of the ASM or whether the contractile elements within asthmatic ASM cells are altered in a manner in which they are less labile and thus, more able to maintain force following mechanical perturbation (or whether it is a combination of both factors). Further investigation of the quantity of myosin filaments using electron microscopy and the proportion of myosin molecular isoforms could provide important insight $[44,45]$.

In conclusion, this study provides valuable information on whether differences in asthmatic ASM mechanical properties underlie airway hyperresponsiveness. It is the first study to demonstrate that asthmatic ASM undergoes length adaptation and responds differently to a mechanical perturbation. We have demonstrated that the mechanical properties of asthmatic ASM may contribute to AHR by an altered length-force relationship following length adaptation and an altered responsiveness to mechanical perturbation which may be due to an increase in stiffness in asthmatic ASM. Further, we suggest that the maximal force generating capacity, shortening velocity, and shortening capacity, are likely not significant contributors to AHR. 


\subsection{References}

1. An SS, Bai TR, Bates JH, Black JL, Brown RH, Brusasco V, Chitano P, Deng L, Dowell M, Eidelman DH, Fabry B, Fairbank NJ, Ford LE, Fredberg JJ, Gerthoffer WT, Gilbert SH, Gosens R, Gunst SJ, Halayko AJ, Ingram RH, Irvin CG, James AL, Janssen LJ, King GG, Knight DA, Lauzon AM, Lakser OJ, Ludwig MS, Lutchen KR, Maksym GN, Martin JG, Mauad T, McParland BE, Mijailovich SM, Mitchell HW, Mitchell RW, Mitzner W, Murphy TM, Pare PD, Pellegrino R, Sanderson MJ, Schellenberg RR, Seow CY, Silveira PS, Smith PG, Solway J, Stephens NL, Sterk PJ, Stewart AG, Tang DD, Tepper RS, Tran T, Wang L. Airway smooth muscle dynamics: a common pathway of airway obstruction in asthma. Eur Respir J 2007;29:834-860.

2. Bai TR. Abnormalities in airway smooth muscle in fatal asthma. A comparison between trachea and bronchus. Am Rev Respir Dis 1991;143:441-443.

3. Cerrina J, Le Roy Ladurie M, Labat C, Raffestin B, Bayol A, Brink C. Comparison of human bronchial muscle responses to histamine in vivo with histamine and isoproterenol agonists in vitro. Am Rev Respir Dis 1986;134:57-61.

4. Cerrina J, Labat C, Haye-Legrande I, Raffestin B, Benveniste J, Brink C. Human isolated bronchial muscle preparations from asthmatic patients: effects of indomethacin and contractile agonists. Prostaglandins 1989;37:457-469.

5. Goldie RG, Spina D, Henry PJ, Lulich KM, Paterson JW. In vitro responsiveness of human asthmatic bronchus to carbachol, histamine, beta-adrenoceptor agonists and theophylline. Br J Clin Pharmacol 1986;22:669-676.

6. Roberts JA, Raeburn D, Rodger IW, Thomson NC. Comparison of in vivo airway responsiveness and in vitro smooth muscle sensitivity to methacholine in man. Thorax 1984;39:837-843.

7. Whicker SD, Armour CL, Black JL. Responsiveness of bronchial smooth muscle from asthmatic patients to relaxant and contractile agonists. Pulm Pharmacol 1988;1:25-31.

8. Bjorck T, Gustafsson LE, Dahlen SE. Isolated bronchi from asthmatics are hyperresponsive to adenosine, which apparently acts indirectly by liberation of leukotrienes and histamine. Am Rev Respir Dis 1992;145:1087-1091.

9. Bramley AM, Thomson RJ, Roberts CR, Schellenberg RR. Hypothesis: excessive bronchoconstriction in asthma is due to decreased airway elastance. Eur Respir $J$ 1994; 7:337-341.

10. Bai TR. Abnormalities in airway smooth muscle in fatal asthma. Am Rev Respir Dis 1990;141:552-557. 
11. de Jongste JC, Mons H, Bonta IL, Kerrebijn KF. In vitro responses of airways from an asthmatic patient. Eur J Respir Dis 1987;71:23-29.

12. Schellenberg RR, Foster A. In vitro responses of human asthmatic airway and pulmonary vascular smooth muscle. Int Arch Allergy Appl Immunol 1984;75:237-241.

13. Thomson RJ, Bramley AM, Schellenberg RR. Airway muscle stereology: implications for increased shortening in asthma. Am J Respir Crit Care Med 1996;154:749-757.

14. Chin LY, Bosse Y, Jiao Y, Solomon D, Hackett TL, Pare PD, Seow CY. Human airway smooth muscle is structurally and mechanically similar to that of other species. Eur Respir J 2009.

15. King GG, Pare PD, Seow CY. The mechanics of exaggerated airway narrowing in asthma: the role of smooth muscle. Respir Physiol 1999;118:1-13.

16. Black JL, Johnson PR. Airway smooth muscle in asthma. Respirology 1996;1:153158.

17. Seow CY, Fredberg JJ. Historical perspective on airway smooth muscle: the saga of a frustrated cell. J Appl Physiol 2001;91:938-952.

18. Seow CY, Schellenberg RR, Pare PD. Structural and functional changes in the airway smooth muscle of asthmatic subjects. Am J Respir Crit Care Med 1998;158:S179-86.

19. Stephens NL, Seow CY, Kong SK. Mechanical properties of sensitized airway smooth muscle. Shortening capacity. Am Rev Respir Dis 1991;143:S13-4.

20. Stephens NL, Halayko AJ. Airway smooth muscle contractile, regulatory and cytoskeletal protein expression in health and disease. Comp Biochem Physiol B Biochem Mol Biol 1998;119:415-424.

21. Ma X, Cheng Z, Kong H, Wang Y, Unruh H, Stephens NL, Laviolette M. Changes in biophysical and biochemical properties of single bronchial smooth muscle cells from asthmatic subjects. Am J Physiol Lung Cell Mol Physiol 2002;283:L1181-9.

22. Solway J, Fredberg JJ. Perhaps airway smooth muscle dysfunction contributes to asthmatic bronchial hyperresponsiveness after all. Am J Respir Cell Mol Biol 1997;17:144-146.

23. King GG, Moore BJ, Seow CY, Pare PD. Airway narrowing associated with inhibition of deep inspiration during methacholine inhalation in asthmatics. Am J Respir Crit Care Med 2001;164:216-218.

24. An SS, Fredberg JJ. Biophysical basis for airway hyperresponsiveness. Can J Physiol Pharmacol 2007;85:700-714. 
25. Hackett TL, Shaheen F, Johnson A, Wadsworth S, Pechkovsky DV, Jacoby DB, Kicic A, Stick SM, Knight DA. Characterization of side population cells from human airway epithelium. Stem Cells 2008;26:2576-2585.

26. Seow CY, Stephens NL. Force-velocity curves for smooth muscle: analysis of internal factors reducing velocity. Am J Physiol 1986;251:C362-8.

27. Hill AV. The heat of shortening and the dynamic constants of muscle. Proceedings of the Royal Society of London 1938;126:136-195.

28. Herrera AM, McParland BE, Bienkowska A, Tait R, Pare PD, Seow CY. 'Sarcomeres' of smooth muscle: functional characteristics and ultrastructural evidence. $J$ Cell Sci 2005; 118:2381-2392.

29. Bai TR, Bates JH, Brusasco V, Camoretti-Mercado B, Chitano P, Deng LH, Dowell M, Fabry B, Ford LE, Fredberg JJ, Gerthoffer WT, Gilbert SH, Gunst SJ, Hai CM, Halayko AJ, Hirst SJ, James AL, Janssen LJ, Jones KA, King GG, Lakser OJ, Lambert RK, Lauzon AM, Lutchen KR, Maksym GN, Meiss RA, Mijailovich SM, Mitchell HW, Mitchell RW, Mitzner W, Murphy TM, Pare PD, Schellenberg RR, Seow CY, Sieck GC, Smith PG, Smolensky AV, Solway J, Stephens NL, Stewart AG, Tang DD, Wang L. On the terminology for describing the length-force relationship and its changes in airway smooth muscle. J Appl Physiol 2004;97:2029-2034.

30. McParland BE, Macklem PT, Pare PD. Airway wall remodeling: friend or foe? J Appl Physiol 2003;95:426-434.

31. Pare PD, McParland BE, Seow CY. Structural basis for exaggerated airway narrowing. Can J Physiol Pharmacol 2007;85:653-658.

32. Jiang H, Rao K, Halayko AJ, Kepron W, Stephens NL. Bronchial smooth muscle mechanics of a canine model of allergic airway hyperresponsiveness. J Appl Physiol 1992;72:39-45.

33. Ammit AJ, Armour CL, Black JL. Smooth-muscle myosin light-chain kinase content is increased in human sensitized airways. Am J Respir Crit Care Med 2000;161:257263.

34. Ishida K, Pare PD, Hards J, Schellenberg RR. Mechanical properties of human bronchial smooth muscle in vitro. J Appl Physiol 1992;73:1481-1485.

35. Opazo Saez AM, Schellenberg RR, Ludwig MS, Meiss RA, Pare PD. Tissue elastance influences airway smooth muscle shortening: comparison of mechanical properties among different species. Can J Physiol Pharmacol 2002;80:865-871.

36. Fish JE, Peterman VI, Cugell DW. Effect of deep inspiration on airway conductance in subjects with allergic rhinitis and allergic asthma. J Allergy Clin Immunol 1977;60:4146. 
37. Fish JE, Kehoe TJ, Cugell DW. Effect of deep inspiration on maximum expiratory flow rates in asthmatic subjects. Respiration 1978;36:57-63.

38. Lim TK, Pride NB, Ingram RH,Jr. Effects of volume history during spontaneous and acutely induced air-flow obstruction in asthma. Am Rev Respir Dis 1987;135:591-596.

39. Lim TK, Ang SM, Rossing TH, Ingenito EP, Ingram RH,Jr. The effects of deep inhalation on maximal expiratory flow during intensive treatment of spontaneous asthmatic episodes. Am Rev Respir Dis 1989;140:340-343.

40. Mauad T, Silva LF, Santos MA, Grinberg L, Bernardi FD, Martins MA, Saldiva PH, Dolhnikoff M. Abnormal alveolar attachments with decreased elastic fiber content in distal lung in fatal asthma. Am J Respir Crit Care Med 2004;170:857-862.

41. James AL. Peripheral airways in asthma. Curr Allergy Asthma Rep 2002;2:166-174.

42. Carroll N, Elliot J, Morton A, James A. The structure of large and small airways in nonfatal and fatal asthma. Am Rev Respir Dis 1993;147:405-410.

43. Bramley AM, Roberts CR, Schellenberg RR. Collagenase increases shortening of human bronchial smooth muscle in vitro. Am J Respir Crit Care Med 1995;152:15131517.

44. Kuo KH, Wang L, Pare PD, Ford LE, Seow CY. Myosin thick filament lability induced by mechanical strain in airway smooth muscle. J Appl Physiol 2001;90:18111816.

45. Leguillette R, Laviolette M, Bergeron C, Zitouni N, Kogut P, Solway J, Kachmar L, Hamid Q, Lauzon AM. Myosin, transgelin, and myosin light chain kinase: expression and function in asthma. Am J Respir Crit Care Med 2009;179:194-204. 


\section{CHAPTER 6: CONCLUSIONS AND FUTURE DIRECTIONS}

In this thesis, I investigated the mechanical, ultrastructural, and biochemical properties of airway smooth muscle at the cellular and tissue levels in health and disease. The first study, in Chapter Two, was focused on the role of the actin filament lattice in myosin filamentogenesis, while the results did not clarify whether the actin filament lattice facilitates myosin filamentogenesis, the study did demonstrate that cytochalasin D likely operates through an unknown mechanism to reduce force but not actin filaments in in situ smooth muscle cells, contrary to findings from cultured cells. In Chapter Three, I examined the functional and structural differences of two contractile agonists: potassium chloride and acetylcholine. This study provided evidence that smooth muscle cells are capable of producing significant force despite a reduction in myosin filaments when stimulated by $\mathrm{KCl}$. These two studies were focused on the contractile apparatus and the mechanisms by which myosin filaments assemble during contraction.

\subsection{Myosin Filamentogenesis and the Contractile Apparatus}

Since myosin filaments disassociate in vitro $[1,2]$ when subjected to physiological $\mathrm{pH}$, $\mathrm{Mg}^{2+}$-ATP concentration, and ionic strength, it is unclear why myosin filaments are even seen in relaxed muscle cells fixed for EM. It is likely that there are stabilizing factors that prevent the filaments from disassociating in vivo. I sought to test the hypothesis that this stability is due to the actin filament lattice. This lattice is a seemingly omnipresent collection of thin filaments which outnumber myosin filaments $\sim 30: 1$ in relaxed ASM, 
however, from the data presented in Chapter Two, the question remains unresolved. Cytochalasin D is a purported actin filament antagonist which is thought to bind the barbed $(+)$ end of the actin filament, leading to a net reduction in actin length. However, my results show that cytochalasin D does not significantly disturb the actin filament lattice in in situ airway smooth muscle cells when examined at low concentrations under EM, but dramatically reduces force. At higher concentrations, the actin filament density increased, which could suggest the disruption of filaments by cytochalasin D, such that there was an increased population of shorter filaments. It is clear that the main action of cytochalasin D is on force generating capacity, since low concentrations decrease force without affecting actin filament density. This reduction in force may be through a direct inhibition of the actomyosin interaction, as has been previously demonstrated [3]. Importantly, this study demonstrates that intact muscle strips respond differently to cytochalasin than cultured smooth muscle cells, which demonstrate a significant reduction in stress fibers to low concentrations [4]. Regardless of these results, it would seem pertinent to examine other actin filament disrupters, such as jasplakinolide or latrunculin, in order to adequately test this hypothesis. Technically, it is a difficult hypothesis to test since the extent of actin filament disruption needs to be significant enough to demonstrate a reduction in filaments but subtle enough to prevent drastic cell changes leading to cell dysfunction or full disruption of the cytoskeleton leading to displacement of organelles. Besides the actin filament lattice, other targets should be considered as possible stabilizers of myosin filaments. Caldesmon and calponin have been suggested as chaperone-like proteins, since they are capable of binding both actin 
and myosin. These proteins could act to stabilize myosin filaments as well as 'guide' monomeric myosin into the actin filament lattice during myosin filament assembly.

Given the lack of pharmacological agents that target myosin, it is difficult to design experiments that directly investigate myosin filament assembly and disassembly. In Chapter Three, a mechanism was outlined in which myosin monomers were capable of producing force without forming filaments under $\mathrm{KCl}$ stimulation. While $\mathrm{KCl}$ is a nonphysiological muscle activator, the ability of the muscle to generate substantial force despite having a reduced number of myosin filaments suggests that non-filamentous myosin may contribute to force in vivo. This ability could be an important mechanism that allows for the malleability of the contractile apparatus. It would suggest that filaments disassemble during the initial phase of contraction (since there are more myosin filaments in relaxed muscle than $\mathrm{KCl}$ activated muscle), in order to optimally form new myosin filaments.

The observation that $\mathrm{KCl}$ activation produces significant force with few filaments, could provide a model to determine whether differential signaling pathways are employed to form filaments. Possible candidate pathways include the downstream signals from diacylglycerol activation and the p38 MAPK pathway (discussed in Chapter Three). Along these lines, it would be interesting to use other contractile agonists that do not operate through G-protein coupled receptors, to determine whether filaments are formed and whether this is due to differential signaling. One such candidate would be phorbol esters, which are known to cause contraction through protein kinase $\mathrm{C}$ activation. 
Likewise, $\mathrm{KCl}$ contraction could be studied further using various pharmacological agents that inhibit certain kinases in the contraction signaling pathway. It is, however, timeconsuming to correlate function with ultrastructure because of the protocol for electron microscopy. Ideally, it would be of interest to find a kinase inhibitor that would reduce filament stability in ACh contracted muscle and then determine whether there were fewer filaments. Also, it would be important to demonstrate 'recovery' of myosin filaments following $\mathrm{KCl}$ stimulation by activating alternative pathways. Perhaps a quicker, cruder, method of determining whether myosin filaments are formed or dissolved during activation, would be to employ the use of birefringence, a method that has been shown to be capable of observing filamentogenesis within intact (non-fixed) tissue [5]. This would allow for greater flexibility in examining filamentogenesis while treating the muscle with pharmacological agents and different contractile agonists. A first step to determine whether $\mathrm{KCl}$ and $\mathrm{ACh}$ cause differences in myosin filamentogenesis through differential cell signaling would be to compare phosphorylation levels of various proteins between the two groups through western blotting. A general outline of which proteins are phosphorylated to a greater or lesser extent in each group would provide possible candidate proteins. From there a process of intervention with pharmacological agents and subsequent observations with birefringence could provide valuable insight.

\subsection{Human ASM and Asthma Pathogenesis}

The last two studies of this thesis focused on characterizing the mechanical properties of human airway smooth muscle - an area of research that has been limited due to the 
difficulty in obtaining good quality human tissue samples. The first study, described in Chapter Four, constitutes a comparison of the mechanical properties of human ASM with ASM from other mammals and with the results from previous human studies. The results demonstrated that human ASM is indeed similar to that of other mammalian species. The study also demonstrated that the use of a high quality tissue source and/or the setting of in situ muscle length as an experimental starting point, increased the similarities between human ASM and mammalian muscle compared to previous studies. These previous studies, examined non-asthmatic and asthmatic human ASM tissue removed by resection, using $\mathrm{L}_{\max }$ (see Chapter Four for definition) as the equilibrated length. The major finding of the study in Chapter Four was that human ASM is capable of substantial shortening, comparable to the $\sim 70 \%$ shortening seen in ASM from other mammalian species, as opposed to $\sim 30 \%$ shown in previous human studies [6]. This difference is likely due to tissue quality and the use of $\mathrm{L}_{\max }$ as the equilibrated length. This was also the first study to demonstrate that human ASM is capable of length adaptation.

Using the same methodology as Chapter Four, these non-asthmatic ASM properties were compared with asthmatic ASM (Chapter Five). While the study did not demonstrate differences in force generating capacity, shortening capacity, or velocity of shortening, there were notable differences in the length-tension properties, especially after a period of length adaptation. Intriguingly, asthmatic ASM was found to respond differently to length oscillation compared to non-asthmatic ASM, presumably due to differences in stiffness between the two groups, which is manifested in the differences seen in the length-passive tension relationships. 
These initial studies provide a framework for examining asthmatic ASM at subcellular, cellular, and tissue levels. While the differences observed due to mechanical perturbation by length oscillation may be due to differences in stiffness between non-asthmatic and asthmatic ASM, it would be pertinent to determine whether the contractile units within the muscle cells are involved in determining this quality. It is entirely possible that the increased stiffness, as demonstrated by the increased passive tension among asthmatics (Fig. 5.5), is a property of increased connective tissue surrounding the cells, thus providing some barrier or buffer to mechanical perturbation, protecting the labile contractile units such that they are able to maintain force better in response to length oscillation. However, the organization of the contractile units may be materially different in asthmatics. For instance, increased myosin filament strength or number would likely convey a similar resistance to the effects of length oscillation. Quantifying actin or myosin filaments between asthmatics and non-asthmatics has never been attempted. Another possibility, if the myosin filament count was similar between the two groups, could be an increased rate of filament assembly following perturbation in asthmatic ASM. While this is likely not the case, given that the rate of force recovery is similar in asthmatics and non-asthmatics (Fig. 5.11), it cannot be entirely ruled out. By examining asthmatic and non-asthmatic ASM under EM before oscillation and throughout the recovery period after oscillation, it would be possible to determine whether initial filament count, filament strength, or rate of assembly are responsible for the differential responses to length oscillation. Also, differential expression of myosin isoforms in the tail region of the molecule, may affect the strength of myosin filaments. 
While the study in Chapter Five did not demonstrate differences in force generating capacity or shortening velocity, more experiments would provide greater certainty as to whether or not these properties are different. A post-hoc power analysis of the force data (Chapter Five), determined that nearly 50 donors would be required for the current variability to be considered statistically significant. While a larger sample size may provide statistical significance, it is unclear whether any differences would be physiologically significant. For shortening velocity, more data is needed at the low load $5 \% \mathrm{~F}_{\max }$ region in order to observe any differences in unloaded maximal velocity $\left(\mathrm{V}_{\max }\right)$. Protein analysis could provide further insight. Changes to the expression of myosin isoforms, perhaps to a faster cycling crossbridge, or increased expression of myosin light chain kinase, could lead to differences in shortening velocity. While increases in myosin heavy chain content could suggest increased force generating capacity.

The importance of this high quality tissue source cannot be understated. By providing a reliable means to compare asthmatic ASM with non-asthmatic ASM, we may finally determine whether asthmatic ASM properties are intrinsically altered with disease. Without mechanical data to support the notion of 'defective' ASM in asthmatics, much of the current focus in asthma research has been on other tissues of the airway (e.g. the epithelial layer) and the immunomodulatory functions of the ASM. In place of the previous paradigm in which ASM cells were thought to only be recipients of cytokines and chemokines secreted from immune cells, the ASM cell has been shown to be a rich source of immunomodulatory mediators $[7,8]$. However, it is not clear whether this 
secretory potential of cultured ASM cells is relevant in vivo or in asthma. Since the previous mechanical data from asthmatics has not pointed to differences in ASM function (see discussion in Chapter Five), the significant observations seen in Chapter Five take on added importance. The differential response to mechanical perturbation by asthmatic ASM compared to non-asthmatic ASM provides an intriguing mechanism by which asthmatic airways may function differently in vivo. The reduced bronchodilatory response to deep inspirations seen in asthmatics may be a property of the ASM itself, suggesting that the ASM is indeed altered in asthmatics. If this is the case, this important finding may finally provide much needed insight into the cause of airway narrowing in asthma. 


\subsection{References}

1. Seow CY. Myosin filament assembly in an ever-changing myofilament lattice of smooth muscle. Am.J.Physiol.Cell.Physiol. 2005; 289: 6: C1363-8.

2. Suzuki H, Onishi H, Takahashi, Watanabe S. Structure and function of chicken gizzard myosin. J.Biochem. 1978; 84: 1529-1542.

3. Saito SY, Hori M, Ozaki H, Karaki H. Cytochalasin D inhibits smooth muscle contraction by directly inhibiting contractile apparatus. J.Smooth Muscle Res. 1996; 32: $2: 51-60$.

4. An SS, Laudadio RE, Lai J, Rogers RA, Fredberg JJ. Stiffness changes in cultured airway smooth muscle cells. Am.J.Physiol.Cell.Physiol. 2002; 283: 3: C792-801.

5. Smolensky AV, Ragozzino J, Gilbert SH, Seow CY, Ford LE. Length-dependent filament formation assessed from birefringence increases during activation of porcine tracheal muscle. J. Physiol. 2005; 563 (Pt2): 517-27.

6. Opazo Saez AM, Schellenberg RR, Ludwig MS, Meiss RA, Paré PD. Tissue elastance influences airway smooth muscle shortening: comparison of mechanical properties among different species. Can. J. Physiol. Pharmacol. 2002; 80(9): 865-71.

7. Hirst SJ. Regulation of airway smooth muscle cell immunomodulatory function: role in asthma. Respir.Physiol.Neurobiol. 2003; 137: 309-26.

8. Oliver BG, Black JL. Airway smooth muscle and asthma. Allergol Int. 2006; 55: 21523. 


\section{APPENDIX A: ETHICS APPROVAL}

After ethics review by the UBC Research Ethics Board, it was determined that since the tissue was received from a local abattoir, ethics approval for use was unnecessary. Thus, there is no certificate related to ethics approval for the tissue used in Chapters Two and Three.

A copy of the ethics approval certificate from the UBC Research Ethics Board is provided on the next page for use of the human tissue in Chapters Four and Five. 


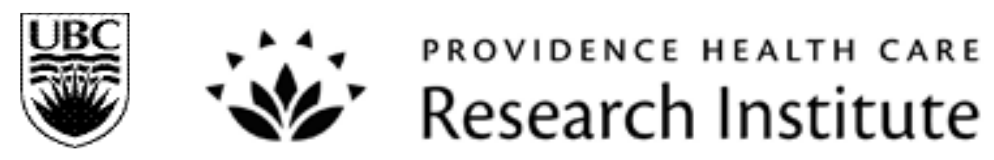

UBC-Providence Health Care Research Institute

Office of Research Services

11th Floor Hornby Site - SPH

c/o 1081 Burrard St.

Vancouver, BC V6Z 1 Y6

Tel: (604) 806-8567

Fax: (604) 806-8568

\section{ETHICS CERTIFICATE OF EXPEDITED APPROVAL: ANNUAL RENEWAL}

\begin{tabular}{|c|c|}
\hline \begin{tabular}{|l|l|} 
PRINCIPAL INVESTIGATOR: & DEPARTMENT: \\
Peter D. Pare & Medicine
\end{tabular} & \begin{tabular}{l|l} 
UBC-PHC REB NUMBER: \\
H00-50110
\end{tabular} \\
\hline \multicolumn{2}{|l|}{ INSTITUTION(S) WHERE RESEARCH WILL BE CARRIED OUT: } \\
\hline Institution & Site \\
\hline $\begin{array}{l}\text { Vancouver Coastal Health (VCHRI/VCHA) } \\
\text { Providence Health Care } \\
\text { Other locations where the research will be conducted: } \\
\text { N/A }\end{array}$ & $\begin{array}{l}\text { Vancouver General Hospital } \\
\text { St. Paul's Hospital }\end{array}$ \\
\hline \multicolumn{2}{|l|}{$\begin{array}{l}\text { CO-INVESTIGATOR(S): } \\
\text { Raja T. Abboud } \\
\text { R. Robert Schellenberg } \\
\text { Don Sin } \\
\text { John Yee } \\
\text { Delbert R. Dorscheid } \\
\text { John C. English } \\
\text { John R. Mayo } \\
\text { Nasreen Khalil } \\
\text { Harvey O. Coxson } \\
\text { Nestor L. Muller } \\
\text { Tony R. Bai } \\
\text { Kenneth G. Evans } \\
\text { Darryl Knight } \\
\text { James C. Hogg } \\
\text { S.F. Paul Man } \\
\text { Stephen Lam } \\
\text { Julia Flint } \\
\text { Richard J. Finley } \\
\end{array}$} \\
\hline $\begin{array}{l}\text { SPONSORING AGENCIES: } \\
\text { Altana Pharma Inc. - "Pulmonary Structure and Function in H } \\
\text { British Columbia Lung Association - "Pulmonary Structure and } \\
\text { Canadian Institutes of Health Research (CIHR) - "Pulmonary } \\
\text { GlaxoSmithKline (Canada) Inc. } \\
\text { GlaxoSmithKline (US) Inc. } \\
\text { National Institutes of Health - "Pulmonary Structure and Func } \\
\text { Science Council of Canada - "Pulmonary Structure and Funct } \\
\text { UBC Operating Budget }\end{array}$ & $\begin{array}{l}\text { h and Disease" } \\
\text { unction in Health and Disease" } \\
\text { ucture and Function in Health and Disease" } \\
\text { in Health and Disease" } \\
\text { in Health and Disease" }\end{array}$ \\
\hline $\begin{array}{l}\text { PROJECT TITLE: } \\
\text { Pulmonary Structure and Function in Health and Disease }\end{array}$ & \\
\hline
\end{tabular}

EXPIRY DATE OF THIS APPROVAL: August 19, 2010

APPROVAL DATE: August 19, 2009

CERTIFICATION:

1. The membership of the UBC-PHC REB complies with the membership requirements for research ethics boards defined in Part C Division 5 of the Food and Drug Regulations of Canada.

2. The UBC-PHC REB carries out its functions in a manner fully consistent with Good Clinical Practices.

3. The UBC-PHC REB has reviewed and approved the research project named on this Certificate of Approval including any associated consent form and taken the action noted above. This research project is to be conducted by the principal investigator named above at the specified research site(s). This review of the UBC-PHC REB have been documented in writing.

|The UBC-PHC Research Ethics Board Chair or Associate Chair, has reviewed the documentation for the above named 
project. The research study, as presented in the documentation, was found to be acceptable on ethical grounds for research involving human subjects and was approved for renewal.

Approval of the UBC-PHC Research Ethics Board or Associate Chair, verified by the signature of one of the following:
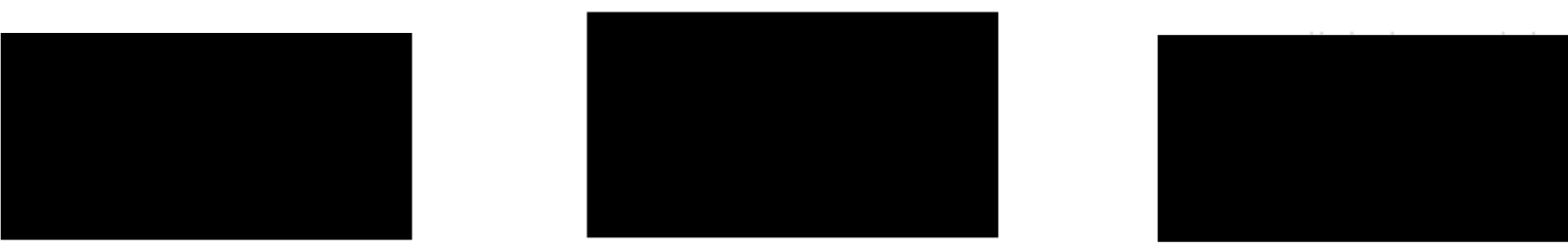